\title{
Degenerate stars and gravitational collapse in AdS/CFT
}

\author{
Xerxes Arsiwalla, ${ }^{a}$ Jan de Boer, ${ }^{a, b}$ Kyriakos Papadodimas $^{c}$ and Erik Verlinde ${ }^{a, b}$ \\ ${ }^{a}$ Institute for Theoretical Physics University of Amsterdam, \\ Amsterdam, The Netherlands \\ ${ }^{b}$ Gravitation and Astro-Particle Physics Amsterdam, \\ Science Park 904, Postbus 94485, 1090 GL, Amsterdam, The Netherlands \\ ${ }^{c}$ Theory Group, Physics Department, CERN, \\ CH-1211 Geneva 23, Switzerland \\ E-mail: x.d.arsiwalla@gmail.com, j.deboer@uva.nl, \\ kyriakos.papadodimas@cern.ch, e.p.verlinde@uva.nl
}

ABSTRACT: We construct composite CFT operators from a large number of fermionic primary fields corresponding to states that are holographically dual to a zero temperature Fermi gas in AdS space. We identify a large $N$ regime in which the fermions behave as free particles. In the hydrodynamic limit the Fermi gas forms a degenerate star with a radius determined by the Fermi level, and a mass and angular momentum that exactly matches the boundary calculations. Next we consider an interacting regime, and calculate the effect of the gravitational back-reaction on the radius and the mass of the star using the Tolman-Oppenheimer-Volkoff equations. Ignoring other interactions, we determine the "Chandrasekhar limit" beyond which the degenerate star (presumably) undergoes gravitational collapse towards a black hole. This is interpreted on the boundary as a high density phase transition from a cold baryonic phase to a hot deconfined phase.

KEYwORDS: AdS-CFT Correspondence, Holography and quark-gluon plasmas, Holography and condensed matter physics (AdS/CMT)

ARXIV EPRINT: 1010.5784 


\section{Contents}

$\begin{array}{llr}1 & \text { Introduction } & 1\end{array}$

2 Free Fermi gas in AdS 5

2.1 Boundary description 5

2.1.1 Degenerate fermionic operators 5

2.1.2 "Free field" mode expansion 9

$\begin{array}{ll}2.1 .3 & \text { Density of states } \\ \end{array}$

2.2 Quantum states in the bulk 11

2.3 Hydrodynamic description $\quad 12$

\begin{tabular}{ll}
2.3 .1 & The equation of state \\
\hline
\end{tabular}

$\begin{array}{lll}\text { 2.3.2 Energy momentum conservation } & 13\end{array}$

$\begin{array}{ll}2.3 .3 & \text { Total mass and particle number } \\ \end{array}$

2.3.4 A comment on the validity of the hydrodynamic description $\quad 15$

$\begin{array}{lll}2.3 .5 & \text { Radial reconstruction } & 16\end{array}$

$\begin{array}{ll}2.3 .6 & \text { Fermions at finite temperature } \\ & 16\end{array}$

$\begin{array}{lll}2.4 & \text { Rotating star } & 17\end{array}$

3 Including interactions $\quad \mathbf{2 0}$

3.1 The scaling limit 20

3.2 Interacting quantum states in AdS 22

$\begin{array}{lll}3.3 & \text { From quantum states to fermionic fluid } 25\end{array}$

3.3.1 The Hartree-Fock approximation 26

3.3.2 Fermion fluid and Thomas-Fermi approximation 28

$\begin{array}{ll}3.4 & \text { TOV equations in the bulk } \\ 3.5 & 28\end{array}$

3.5 Relation between quantum description and TOV approach 30

3.6 Quantum states vs. classical geometries in AdS/CFT 31

$4 \quad$ Numerical solutions and gravitational collapse $\quad 32$

$\begin{array}{lll}4.1 & \text { Numerical results } & 32\end{array}$

4.1.1 A typical fermionic star 33

$\begin{array}{lll}\text { 4.1.2 Families of stars and a critical mass } & 34\end{array}$

4.1.3 Dependence on the fermion mass 36

$\begin{array}{lll}4.2 & \text { The Chandrasekhar limit } & 37\end{array}$

$\begin{array}{lll}4.3 & \text { Endpoint of the collapse } & 38\end{array}$

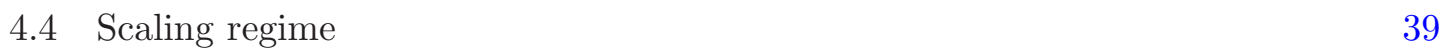

$\begin{array}{lll}\text { 4.4.1 Limiting solution } & 39\end{array}$

$\begin{array}{lll}4.4 .2 & \text { Oscillations of the mass } & 40\end{array}$ 
5.1 Graviton exchange and first correction to anomalous dimensions 42

5.2 Boundary description of the instability and the collapse 45

6 Validity and embedding in string theory 46

6.1 Toy model with a single fermionic field and the graviton 46

$\begin{array}{ll}6.2 & \text { Including other modes }\end{array}$

$\begin{array}{lll}7 & \text { Charged stars } & 49\end{array}$

$\begin{array}{ll}7.1 \text { Charged TOV equations } & 50\end{array}$

$\begin{array}{ll}7.2 \text { Numerics of charged solutions } & 51\end{array}$

$\begin{array}{lll}\text { 7.2.1 A charged degenerate star } & 51\end{array}$

7.2.2 Family of charged TOV solutions and critical mass 53

$\begin{array}{lll}7.2 .3 & \text { Attractor fixed points } & 54\end{array}$

8 Thermal graviton star $\quad 55$

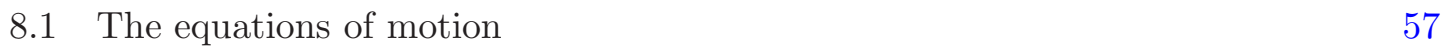

$\begin{array}{ll}\text { 8.2 The graviton star solution } & 59\end{array}$

9 Discussions $\quad 60$

\section{Introduction}

String theory contains all kinds of elementary particles and forces, and thus in principle can be used to describe all forms of matter. In the past two decades much of the progress in string theory has centered around black holes, which may be viewed as the most extreme form of matter. In fact, the matter that makes up a black hole is in such an extreme form that it has entirely disappeared from the picture and is replaced by pure geometry. Holography may be seen as an attempt to rescue some of this matter by assigning material properties to the black hole horizon. To clarify the relationship between the holographic matter and the matter that made the black hole one needs to investigate the process of black hole formation, in other words, gravitational collapse.

A related question is whether string theory, in the context of the AdS/CFT correspondence [1], can be used to describe other extreme forms of matter, such as the degenerate matter that makes up a neutron star. Indeed, neutron stars, or more generally degenerate stars like "quark stars" or "strange stars", can be seen as black hole precursors in the sense that they may undergo gravitational collapse to form a black hole. An essential feature of neutron stars is that they are made out of fermions: it is the exclusion principle that keeps the star from undergoing gravitational collapse. Our aim is to obtain a description of a "neutron star" (or more precisely a "degenerate star") in anti de Sitter space 
in terms of the dual conformal field theory on the boundary. In particular, we are interested to investigate and interpret the criterion that determines whether the star undergoes gravitational collapse.

One of the first and most famous equations that deals with the physics of a neutron star is the Tolman-Oppenheimer-Volkoff (TOV) equation. In four dimensional flat space time it takes the form

$$
\frac{d p(r)}{d r}=-2 G \frac{p(r)+\rho(r)}{r^{2}} \cdot \frac{M(r)+4 \pi r^{2} p(r)}{1-2 G M(r) / r}
$$

where the radial dependent mass $M(r)$ is obtained from the energy density $\rho(r)$ through

$$
\frac{d M(r)}{d r}=4 \pi r^{2} \rho(r)
$$

The TOV equation follows from combining the energy-momentum conservation with the Einstein equations and holds for spherically symmetric neutron stars. It describes the radial dependence of the pressure $p(r)$ and energy density $\rho(r)$ for a given equation of state. Solutions only exist when the pressure is sufficient to sustain the gravitational force to prevent the neutron star from collapsing. Typically one finds that there is an upper limit for the mass beyond which the neutron star will be too heavy and starts to collapse to form a black hole (or in some cases another type of degenerate star). In their original paper [2] Oppenheimer and Volkoff used the equation of state of a free relativistic Fermi gas to find a limiting mass of 0.7 times the solar mass. However, more realistic equations of state lead to a higher value of the limiting mass around 2 or 3 solar masses.

In this paper we construct states in the boundary CFT that are the holographic dual of a degenerate star in anti de Sitter space. Our discussion will be quite general without reference to a specific AdS/CFT setup and the qualitative aspects of our results are independent of the number of space time dimensions. Using the state-operator correspondence we consider degenerate states corresponding to composite multi-trace operators constructed from a large number of copies of a given fermionic single trace operator. Using 't Hooft factorization it is argued that in a strict large $N$ limit these operators behave as (generalized) free fields. The bulk description of these states is given by a zero temperature degenerate gas of relativistic fermions. In the limit of a large number of particles one can give a hydrodynamic description purely in terms of their energy density and pressure. We first consider the case without bulk interactions so that the equation of state is simply that of a free degenerate Fermi gas. We show that in the hydrodynamic description the degenerate star is confined to a spherical region in AdS space, due to the gravitational potential well. The radius in AdS units turns out to be given by the ratio of the Fermi momentum in the center of the star and the mass of the constituent fermions. As a verification of our hydrodynamic description we show that it precisely reproduces the mass predicted from a boundary analysis for static degenerate stars as well as for the case with rotation.

Next we consider a double scaling regime chosen so that the gravitational self interactions of the fermions will become important, while we can still ignore Planck scale physics. Since our primary interest is to understand first qualitatively the criterion for gravitational 
collapse we focus our attention to including the self gravity of the degenerate star, while ignoring the role of all other interactions. Thus, following Oppenheimer and Volkoff, we use the equation of state of a free degenerate Fermi gas in the hydrodynamic description of the star, and take the gravitational self interaction into account through a generalization of the TOV equation to five dimensional anti de Sitter space. The resulting equations are studied numerically. We find that as a function of the central density, the mass of the star at first increases, but reaches a limiting mass at some critical value after which the mass slightly reduces again. We interpret the limiting mass as the point after which the star will become unstable and will start to undergo gravitational collapse. The value of the limiting mass translates in the boundary theory to a limiting conformal dimension of the composite operator made from the fermionic primary fields.

A basic motivation for our work was towards analyzing the process of gravitational collapse and black hole formation in AdS/CFT. This is an interesting problem, which has been addressed in several works in the past [3-16]. The basic intuition is that radial collapse corresponds to motion in the "scale direction" of the CFT, while the subsequent black hole formation corresponds to deconfinement and thermalization on the boundary theory. A more quantitative description of this picture would clearly be desirable, but is difficult since the boundary theory is strongly interacting, the process time dependent and the radial holographic dimension is not easily represented in the CFT. In this direction, it may be helpful to identify simple initial conditions to study (at least the onset of) the dynamical process of collapse. A star, on the verge of gravitational collapse, is a good candidate since it is a static configuration and presumably simpler to understand from the boundary point of view.

Another motivation was to make contact with the recent developments on the holographic analysis of strongly interacting fermions. In several works [17-20] correlators of fermionic fields have been evaluated in the presence of a black hole in AdS. Such computations reveal the presence of a Fermi surface in the bulk, of non-Fermi liquid type. For a better understanding of these results it might be worthwhile to explore the boundary meaning of a simpler system, that of a Fermi-gas in AdS without the presence of a black hole. ${ }^{1}$ One should keep in mind that while the fermions are free in the bulk, they are not ordinary free fermions on the boundary (i.e. they do not obey a Dirac equation on the boundary) as the boundary theory is strongly coupled. Instead they are "generalized free fields" i.e. their correlators factorize even though they do not obey linear wave equations.

Finally let us mention another motivation for constructing a holographic degenerate star, one of a more conceptual nature. Our degenerate star in AdS resembles, for many purposes, a conventional macroscopic object. Since it is embedded in AdS it has a holographic description on the boundary theory. It would be interesting to understand in more detail how the hologram works in this case. In particular it might be easier to "decode" the hologram in the case of a star than for a black hole, due to the absence of a horizon. Our star is a static, spherically symmetric configuration in the bulk. The only nontrivial

\footnotetext{
${ }^{1}$ The interesting paper [21] appeared while this draft was being prepared, in which fermion correlators were evaluated on a background with fermions, without a black hole horizon.
} 
information is encoded in the radial profile of the density. It is not obvious how this radial profile is encoded in the boundary theory, since the radial dimension is not manifest on the boundary but rather entangled with the "scale" of excitations in the CFT. We believe that a star in AdS is a good toy model to further explore the holographic mapping.

We would like to emphasize the following point about the aforementioned goal. One might think that decoding the hologram on the boundary theory is difficult because of strong coupling effects in the gauge theory and thus hopeless unless one manages to compute at strong coupling. While this is true to some extent, we believe that there are interesting questions to be understood, which are not dependent on strong coupling dynamics. In a large $N$ gauge theory the strong coupling effects can be "hidden" in the conformal dimensions and 3-point functions of single-trace gauge invariant operators. These quantities are difficult to compute. Nevertheless, let us assume that in some magical way we knew their exact values at strong coupling. Would that be sufficient to understand how the hologram works? In principle it should, ${ }^{2}$ because from this information we can reproduce all correlators of the CFT. However in practice, even armed with this information, we do not know yet how to reconstruct the bulk. Understanding how the radial profile of a macroscopic object, such as our degenerate star, is encoded on the boundary theory may be a good starting point for addressing this question. Ultimately it would be fascinating to understand the CFT meaning of the Einstein/TOV equations. ${ }^{3}$ Unfortunately we do not have much to report on this yet but we hope to revisit this and related questions in future work.

The main idea presented in this work was briefly described in [22]. In this paper we expand on various aspects that were not covered in detail in [22], we consider the case of charged fermions and that of a thermal star, and finally we discuss some issues about the validity of our approximations and the embedding in specific AdS/CFT dualities. The plan of the paper is as follows: in section 2 we consider a ball of Fermi gas in AdS in the limit of infinite $N$ and construct its dual holographic representation. In section 3 we discuss our double scaling limit, estimate the importance of various interactions and present the TOV equations for the fermionic fluid. In section 4 we present our numerical results and discuss the Chandrasekhar limit beyond which the star undergoes gravitational collapse. In section 5 we try to understand the boundary interpretation of the gravitational interactions between the fermions. In section 6 we discuss the extent to which our various approximations are justified and the possibility of embedding our construction in a specific AdS/CFT correspondence. In section 7 we analyze the case where the fermions are charged under a $\mathrm{U}(1)$ gauge field in the bulk. In section 8 we present a similar system corresponding to a star made out of thermal gas and discuss its relevance as the dual of a "superheated phase" in certain gauge theories. Finally in section 9 we close with some discussions.

\footnotetext{
${ }^{2}$ At least for length scales which are not too small compared to the size of AdS and in the absence of black holes. In other words when the $\mathcal{O}\left(N^{2}\right)$ degrees of freedom are not excited nontrivially above their ground state at strong coupling.

${ }^{3}$ In CFTs with classical gravity dual.
} 


\section{Free Fermi gas in AdS}

In this section we will study a degenerate gas of free fermions in $\operatorname{AdS}_{d+1}$. We will assume that the bulk theory is holographically dual to a conformal field theory which has the analogue of a large $N$ expansion. To be more general we will express the expansion parameter in terms of the central charge $c$ of the CFT. In this section we work in the limit $c \rightarrow \infty$, keeping other quantities fixed. In this limit all interactions between single-trace operators are suppressed by powers of $1 / \sqrt{c}$ and the fermions become essentially free. The only interaction which remains is the gravitational attraction of the AdS background which acts as a confining box. We work in a regime of the gauge theory where there is a classical gravity dual, such as the $\lambda \gg 1$ limit in the $\mathcal{N}=4$ SYM. $^{4}$

\subsection{Boundary description}

We will construct composite multi-trace operators that are made out of a large number of single trace fermionic operators. The composite operators will be "degenerate" in the sense that they represent the operators with the lowest possible conformal dimension that can be made out of a given number of single trace fermionic operators of a certain kind. Through the state-operator correspondence these degenerate composites represent the states that are holographically dual to the degenerate fermionic gas in the bulk theory.

\subsubsection{Degenerate fermionic operators}

Gravity in $\mathrm{AdS}_{d+1}$ is holographically dual to a conformal field theory on $S^{d-1} \times$ time. The Hilbert spaces of the two theories are isomorphic, so any quantum state in the bulk is dual to a state in the CFT. The states of the CFT can be conveniently labeled by local operators on the plane via the state-operator correspondence

$$
\lim _{z \rightarrow 0} \Phi(z)|\operatorname{vac}\rangle=|\Phi\rangle
$$

The energy of the state $|\Phi\rangle$ is equal to $\Delta / \ell$ where $\ell$ is the radius of $S^{d-1}$ and $\Delta$ the dimension of the operator $\Phi$. The other quantum numbers of the state (like the angular momentum and R-charge) are also determined by those of $\Phi$.

In AdS/CFT one makes a distinction between single trace and multi trace operators. The first kind may be thought of as single particle states, while the latter are multiparticle states. However, this distinction is only well defined at large $N$, or more generally for certain classes of CFTs with a large central charge $c$. The reason being that $1 / N$ effects will lead to a mixing between single trace and multi-trace operators.

For certain strongly coupled supersymmetric CFTs the bulk theory is given by an AdS supergravity theory, which for large central charge can be treated (semi-) classically. The bulk theory contains bosons and fermions, and accordingly the boundary single trace operators may be distinguished into bosonic and fermionic operators. Whether a field is fermionic or bosonic is determined according to the spin-statistics theorem through its

\footnotetext{
${ }^{4}$ However, as we will discuss in section 6 , the $\mathcal{N}=4 \mathrm{SYM}$ may not be the best setup to realize the degenerate star.
} 
representations of the $\mathrm{SO}(d, 2)$ conformal symmetry group. In particular, primary operators that transform in the spinor representation correspond to fermions, and have correlation functions that are anti-symmetric under the exchange of two identical operators.

The fermionic nature of primary operators can in specific cases also be understood from their construction in terms of the basic fields of the underlying field theory. To give a concrete example, let us consider the case of $\mathcal{N}=4 \mathrm{SYM}$. The spectrum of operators contains chiral primary fields that are holographically dual to the Kaluza-Klein modes of the type IIB supergravity theory on $\mathrm{AdS}_{5} \times \mathrm{S}^{5}$. These chiral primaries form representations of the $\mathrm{SO}(6) \mathrm{R}$-symmetry group, and are arranged in short supermultiplets. The lowest component is given by the bosonic single trace operators

$$
\Phi^{I_{1} I_{2} \ldots I_{k}}=\left[\operatorname{tr}\left(\phi^{I_{1}} \phi^{I_{2}} \phi^{I_{3}} \ldots \phi^{I_{k}}\right)\right]_{\substack{\text { symmetric } \\ \text { traceless }}}
$$

By applying a supersymmetry transformation one learns that the same supermultiplet contains fermionic single trace operators of the form

$$
\Psi_{A \alpha}^{I_{1} I_{2} \ldots I_{k}}=\left[Q_{A \alpha}, \Phi^{I_{1} I_{2} \ldots I_{k}}\right]=\left[\Gamma_{A B}^{I_{1}} \operatorname{tr}\left(\lambda_{a}^{B} \phi^{I_{2}} \phi^{I_{3}} \ldots \phi^{I_{k}}\right)\right]_{\substack{\text { symmetric } \\ \text { traceless }}}
$$

Here $\Gamma_{A B}^{I}$ is an $\mathrm{SO}(6)$ gamma matrix, with spinor indices $A, B$ and vector index $I$. We note that this operator is not primary with respect to the superconformal group, but it is a conformal primary operator with respect to its bosonic subgroup. The BPS bound implies that the field $\Psi$ has conformal dimension $k+\frac{1}{2}$ for all values of the 't Hooft coupling $\lambda$. The fermionic field $\Psi$ also carries an $\mathrm{SO}(4)$ spinor index $\alpha$, and a multi-index made from a symmetric traceless combination of vector indices. In the following we clearly wish to avoid using all of these indices. Indeed, except for the fact that these fields are fermionic, we have no need for any of these quantum numbers. This is because we will work either in a regime in which we ignore all interactions, or in an approximation in which we do the same except for gravity. ${ }^{5}$ All this will not be very relevant for what follows, but merely was meant to give a concrete example of a fermionic primary field.

Let us now start with a fermionic single trace operator $\Psi$ of dimension $\Delta_{0}$. For simplicity we ignore the spinor indices of $\Psi$ since this will not affect any of our following results qualitatively. We emphasize that $\Psi$ is not one of the fundamental field of the Lagrangian but rather a gauge invariant operator which has finite conformal dimension at strong coupling. We work in normalization where the 2-point function is

$$
\langle\Psi(x) \bar{\Psi}(y)\rangle=\frac{1}{|x-y|^{2 \Delta_{0}}}
$$

The 't Hooft large $c$ factorization implies that for single trace operators the connected correlators are suppressed as

$$
\left\langle\Psi\left(x_{1}\right) \Psi\left(x_{2}\right) \ldots \Psi\left(x_{n}\right)\right\rangle_{c o n}=\mathcal{O}\left(c^{\frac{2-n}{2}}\right)
$$

\footnotetext{
${ }^{5}$ And even if we would try to keep track of R-charges, we can still construct neutral composite states by including all fermionic operators in a complete multiplet. The only complication this will lead to is a degeneracy factor equal to the dimension $g$ of the $\mathrm{SO}(6)$ representation corresponding to the traceless symmetric $s$-tensors.
} 
which in turn implies that the field $\Psi(x)$ behaves effectively like a "free" field at large $c$. We can construct multi trace operators by taking products of many of these fields without having to worry about operator mixing with operators with a different number of traces. In a sense, at large $c$ the fermionic operators generate a Fock space, just like free fields. ${ }^{6}$ This fact will be true as long as the number of operators, or more precisely the total conformal dimension, is small compared to the central charge $c$ of the CFT. This point will become important when we discuss the possible role of interactions.

We now turn to the multitrace operators that can be constructed from the operator $\Psi$. From the fact that correlation functions are antisymmetric under the exchange of two fermionic primaries

$$
\langle\ldots \Psi(x) \Psi(y) \ldots\rangle=-\langle\ldots \Psi(y) \Psi(x) \ldots\rangle
$$

one derives that the operator product of two of these primaries is also antisymmetric

$$
\Psi(x) \Psi(y)=-\Psi(y) \Psi(x) .
$$

Normally one defines the normal ordered product : $\Psi^{2}(x)$ : by taking the limit $x \rightarrow y$ after subtracting possible divergent terms. For fermionic fields the result is zero, due to the antisymmetry. This is of course just a reflection of the Pauli exclusion principle. Therefore, to construct a nontrivial operator out of two fermionic primaries we have to include a derivative. This gives operators of the kind : $\Psi \partial_{i} \Psi$ :, where $\partial_{i}$ denotes the derivative w.r.t. $x_{i}$. This operator is the operator of lowest conformal dimension that can be found in the regular part of the operator product expansion of $\Psi(x) \Psi(y){ }^{7}$ For a $d$-dimensional CFT there are $d$ types of these operators.

We want to continue with the construction of multi-trace operators of the lowest possible conformal dimension made out of three, four, all the way to a very large number of copies of the basic fermionic operator $\Psi$. Each time we have to consider the fact that the next operator needs to be anti-symmetrized with the previous ones. So we have to act again with derivatives every time making sure that the combination of derivatives has not been used before and also that we are using the smallest number of derivatives that are necessary. If we have constructed an operator of this type with $N_{F}$ insertions of the basic operator $\Psi$, we can inductively define the degenerate operator with $N_{F}+1$ insertions as the operator with lowest conformal dimension appearing in the regular part of the OPE between the composite with $N_{F}$ insertions of $\Psi$ 's and one additional $\Psi$. This definition is unambiguous at infinite $c$. Continuing like this one notices that one is filling up "shells", very much like an atom, where each shell is labeled with the number $n$ of derivatives that are being used.

In order to end up with a rotationally symmetric composite operator it is natural to consider the "noble elements" with a completely filled last shell. We will denote the number of derivatives in the last shell by $n_{F}$, since it plays the role of a Fermi level. In this way

\footnotetext{
${ }^{6}$ Though it is not a standard free field in $d$ dimensions, but rather a "generalized free field" [23]: it does not obey any linear wave equation since its conformal dimension is non-canonical but nevertheless its correlators factorize.

${ }^{7}$ This statement is precise in the $c \rightarrow \infty$ limit.
} 
we arrive at the following form of the degenerate composite operators

$$
\boldsymbol{\Phi}=\Psi \prod_{i} \partial_{i} \Psi \prod_{\{i, j\}} \partial_{i} \partial_{j} \Psi \prod_{\{i, j, k\}} \partial_{i} \partial_{j} \partial_{k} \Psi \ldots \ldots \prod_{\left\{i_{1}, i_{2}, \ldots i_{n}\right\}} \partial_{i_{1}} \partial_{i_{2}} \ldots \partial_{i_{n_{F}}} \Psi
$$

where one takes in each shell the product over all possible $n$-tuples made from $d$ derivatives. We note that the composite operator only depends on the choice of the number $n_{F}$. It does not carry any other indices, and hence the corresponding state is unique. In other words, we do not have to deal with counting states: the total entropy is equal to zero. It should also be obvious why these composite operators are called "degenerate".

In the bulk interpretation each insertion of the operator $\Psi$ represents the addition of a particle. Some simple combinatorics show that a completely filled shell made from fields with $n$ derivatives contains

$$
\left(\begin{array}{c}
n+d-1 \\
d-1
\end{array}\right)
$$

different fields, and hence corresponds to that many particles in the bulk. The total number of fields (or particles) is found by summing over all shells

$$
N_{F}=\sum_{n=0}^{n_{F}}\left(\begin{array}{c}
n+d-1 \\
d-1
\end{array}\right)=\left(\begin{array}{c}
n_{F}+d \\
d
\end{array}\right)
$$

Next let us now determine the conformal dimension of a degenerate composite operator $\mathbf{\Phi}$ with $n_{F}$ filled shells. The conformal dimension of the operators in the $n$-th shell is $n+\Delta_{0}$, since each derivative adds one to the conformal dimension. Hence the total conformal dimension is

$$
\Delta=\sum_{n=0}^{n_{F}}\left(n+\Delta_{0}\right)\left(\begin{array}{c}
n+d-1 \\
d-1
\end{array}\right)=d\left(\begin{array}{c}
n_{F}+d \\
d+1
\end{array}\right)+\Delta_{0}\left(\begin{array}{c}
n_{F}+d \\
d
\end{array}\right)
$$

The second term is proportional the the total number of fermions and represents the contribution of their "rest mass" to the energy, while the first term is the "kinetic energy" coming from the derivatives.

In this discussion we have ignored so far the spinor index of the field. This can be treated in a similar way as the electron spin in atomic physics. It simply tells us that each state can be occupied by a number of fermions, namely as many as the dimension of the spinor representation. So if we would have included it, we would have to write an additional product over $\alpha$ which would have multiplied the counting formulas for $N_{F}$ and $\Delta$ by the dimension of the spinor representation. Similarly, if we would have considered a number of species $g$ of fermions, these formulas have an overall factor $g$.

Apart from these harmless simplifications we would like to emphasize that our discussion so far has been exact for all values of the coupling of the gauge theory, as long as $c \rightarrow \infty$. We have assumed that the basic building block $\Psi$ of our operators has conformal dimension $\Delta_{0}$. At the moment we do not care whether this $\Delta_{0}$ is the same as the conformal dimension of $\Psi$ at weak coupling. For us $\Delta_{0}$ can be considered as an input parameter which is determined by the (possibly strongly coupled) dynamics. But even 
this issue could be settled by taking $\Psi$ to be a chiral primary in a supersymmetric theory. On the other hand the operator $\boldsymbol{\Phi}$ defined in (2.2) is definitely not supersymmetric, even if $\Psi$ is, since the derivatives increase the conformal dimension without adding R-charge. The reason that the expression (2.4) is still reliable is based on the 't Hooft factorization at large $c$ : the conformal dimensions of multi-trace operators are equal to the sum of the individual conformal dimensions up to $\frac{1}{c}$ corrections. This statement is based on the planar expansion and does not depend on the value of the 't Hooft coupling. Hence in the limit $c \rightarrow \infty$ our formula (2.4) is exact. The 't Hooft factorization also guarantees that at large $c$ we do not have mixing of the operator $\boldsymbol{\Phi}$ with other operators with the same quantum numbers. These statements are reliable if we take $c \rightarrow \infty$ keeping other quantities (like $\Delta_{0}$ and $n_{f}$ ) fixed.

Finally, let us finish this section with a comment on the $\mathrm{SO}(d)$ decomposition of the states in the $n$-th shell. These states are given by symmetric $n$-tensors, but since they are not traceless they do not form an irreducible representation. The dimension of the irreducible representation of the symmetric traceless $n$-tensors is given by the number of symmetric $n$-tensors minus the number of symmetric $(n-2)$-tensors. In this way we find that the states in the $n$-th shell decompose into a sum of representations corresponding to traceless symmetric $(n-2 k)$-tensors, with $k$ an integer that runs from ${ }^{8} 0$ to $[n / 2]$. Just as a check one verifies that

$$
\left(\begin{array}{c}
n+d-1 \\
d-1
\end{array}\right)=\sum_{k=0}^{[n / 2]}\left[\left(\begin{array}{c}
n-2 k+d-1 \\
d-1
\end{array}\right)-\left(\begin{array}{c}
n-2 k+d-3 \\
d-1
\end{array}\right)\right]
$$

\subsection{2 "Free field" mode expansion}

The state-operator correspondence is a convenient way to describe states in a CFT. The relationship with the corresponding bulk state becomes more manifest, however, in a slightly different representation of the states. Instead of creating the state at $\tau=-\infty$ one can represent the same state by making use of a mode expansion of the primary field on the cylinder $S^{d-1} \times R$. So we go back to Minkowski signature. At large $c$ the primary operator can be expanded in modes [24] on $S^{d-1}$ as

$$
\Psi(t, \Omega)=\sum_{n, l, m} \alpha_{n, l, m} Y_{l}\left(\Omega_{d-1}\right) e^{i E_{n} t}+\beta_{n, l, m}^{\dagger} Y_{l}^{*}\left(\Omega_{d-1}\right) e^{-i E_{n} t}
$$

where the energies satisfy the condition $E_{n l}=\left(\Delta_{0}+n\right) / \ell, n=0,1,2 \ldots$, determined by the representations of the conformal group. The expansion (2.6) has to be understood as a definition of the operators $\alpha_{n, l, m}, \beta_{n, l, m}$. Using the 2-point function of the field $\Psi$ and the fact that its correlators factorize at large $c$ we find that the operators $\alpha_{n, l, m}, \beta_{n, l, m}$ satisfy the algebra of fermionic ladder operators

$$
\left\{\alpha_{n, l, m}, \alpha_{n^{\prime}, l^{\prime}, m^{\prime}}^{\dagger}\right\} \sim \delta_{n n^{\prime}} \delta_{l l^{\prime}} \delta_{m, m^{\prime}} \quad, \quad\left\{\beta_{n, l, m}, \beta_{n^{\prime}, l^{\prime}, m^{\prime}}^{\dagger}\right\} \sim \delta_{n n^{\prime}} \delta_{l l^{\prime}} \delta_{m, m^{\prime}}
$$

and all other anticommutators are zero. The vacuum state satisfies the condition $\alpha_{n, l, m}|v a c\rangle=$ $\beta_{n, l, m}|v a c\rangle=0$. So we can use these modes to build a multi particle state from the vacuum.

\footnotetext{
${ }^{8}$ Here with [ ... ] we denote the integral part of a possibly fractional number.
} 
In this way one finds the state $|\boldsymbol{\Phi}\rangle$ corresponding to the operator (2.2) has an alternative representation as

$$
|\boldsymbol{\Phi}\rangle=\prod_{n, l, m} \alpha_{n, l, m}^{\dagger}|v a c\rangle
$$

However it should be stressed that the expansion (2.6) and the commutation relations (2.7) are only true in the $c \rightarrow \infty$ limit, keeping other quantities fixed. If the operator $\Psi$ is inserted between states whose conformal dimension is of the order of the central charge, then factorization may not hold any more and the commutation relations (2.7) will be modified. Hence the repeated use of the modes of the operator $\Psi$ is only allowed in the limit of large central charge where factorization is reliable.

\subsubsection{Density of states}

Let us now introduce the total mass $M$, the fermion mass $m_{f}$, and the Fermi energy $\epsilon_{F}$ via

$$
m_{f}=\frac{\Delta_{0}}{\ell}, \quad M=\frac{\Delta}{\ell}, \quad \epsilon_{F}=\frac{n_{F}+\Delta_{0}}{\ell} .
$$

where $\ell$ is the radius of the boundary $S^{d-1}$. We express the number of particles $N_{F}$ given in (2.3) and the mass $M$ in terms of the Fermi energy $\epsilon_{F}$ and the fermion mass $m_{f}$ in the limit that $n_{F}=\left(\epsilon_{F}-m_{f}\right) \ell$ and $\Delta_{0}=m_{f} \ell$ become large. The number of fermionic particles may be expressed as

$$
N_{F}=\frac{\ell^{d}}{d !}\left(\epsilon_{F}-m_{f}\right)^{d}
$$

where we ignored all terms that are subleading in $n_{F}$. For the total mass we use the expression (2.4) for the total conformal dimension $\Delta$. Keeping the leading contributions of both terms we find

$$
M=\frac{d \ell^{d}}{(d+1) !}\left(\epsilon_{F}-m_{f}\right)^{d+1}+\frac{m_{f} \ell^{d}}{d !}\left(\epsilon_{F}-m_{f}\right)^{d}
$$

It is important that we keep both $\epsilon_{F}$ as well as $m_{f}$ in these expressions, since we want to keep the ratio of $\epsilon_{F}$ and $m_{f}$ finite. The expressions (2.10) and (2.11) only hold in the specified regime, since we have not taken into account any interactions, gravitational or otherwise.

We will be interested in the limit of large $n_{F}$ (and large $\Delta_{0}$ ), so it is more convenient to rewrite the sums in the expressions (2.3) and (2.4) as integrals. This gives

$$
N\left(\epsilon_{F}\right)=\int_{m_{f}}^{\epsilon_{F}} d \epsilon g(\epsilon), \quad M\left(\epsilon_{F}\right)=\int_{m_{f}}^{\epsilon_{F}} d \epsilon \epsilon g(\epsilon),
$$

where the density of states $g(\epsilon)$ is given by

$$
g(\epsilon)=\frac{\ell^{d}}{(d-1) !}\left(\epsilon-m_{f}\right)^{d-1}
$$

This expression is valid in the strict large $c$ limit. 
Notice that this density of states differs qualitatively from that of a free fermion in $d$ spacetime dimensions. For the latter the density of states grows like $\epsilon^{d-2}$. In other words, while the fermionic operator $\Psi$ is defined in a $d$ dimensional CFT we have found that the density of states created by it grows like that of a $d+1$ dimensional gas. Of course this is related to the fact that the operator $\Psi$ is not really a fundamental free field obeying a linear wave equation, but rather a "generalized free field" which naturally lives in one additional dimension. In a sense this dimension is the scale in the conformal field theory.

\subsection{Quantum states in the bulk}

The fermionic single trace operator $\Psi$ corresponds to a fermionic field $\psi$ in AdS spacetime. On-shell this field satisfies a field equation which in principle depends on the spin of the field. For spin one-half it would be the Dirac equation, or for higher spin one would get the Rarita-Schwinger equations and its generalizations. But since eventually we are interested in taking the limit of a large number of particles and making contact with a hydrodynamic description, the distinction between the different type of fermionic particles and field equations becomes irrelevant. Therefore, to simplify our discussion we will use the partial waves of the Klein-Gordon equation, which are easier to deal with and closer to the standard knowledge. ${ }^{9}$

The global $\mathrm{AdS}_{d+1}$ metric is given by

$$
d s^{2}=-\left(1+\frac{r^{2}}{\ell^{2}}\right) d t^{2}+\left(1+\frac{r^{2}}{\ell^{2}}\right)^{-1} d r^{2}+r^{2} d \Omega_{d-1}^{2} .
$$

where $\ell$ is the AdS radius.

We will assume that the field $\psi$ satisfies the $d+1$-dimensional Klein Gordon equation in AdS space, which in full detail looks like

$$
\left[-\left(1+\frac{r^{2}}{\ell^{2}}\right)^{-1} \partial_{t}^{2}+r^{1-d} \partial_{r}\left(\left(1+\frac{r^{2}}{\ell^{2}}\right) r^{d-1} \partial_{r}\right)+r^{-2} \Delta_{\Omega}+m_{f}^{2}\right] \psi(t, r, \Omega)=0,
$$

where $\Delta_{\Omega}$ denotes the laplacian on $S^{d-1}$. The mass $m_{f}$ of the field $\psi$ and the conformal dimension of the operator $\Delta_{0}$ are related by the formula $\Delta_{0}=\frac{d}{2}+\sqrt{\frac{d^{2}}{4}+\left(m_{f} \ell\right)^{2}}$. For large values of $m_{f}$ we can approximate $\Delta_{0} \approx m_{f} \ell$, which of course was the reason for the identification in (2.9). Since we are interested in states which can be considered as finite energy excitations above the standard vacuum of the theory, we only focus on the normalizable modes in AdS. We then find that the partial wave solutions of the Klein Gordon equation take the general form

$$
\psi_{n, l, \mathbf{m}}(t, r, \Omega)=e^{-i\left(n+\Delta_{0}\right) t / \ell} f_{n-2 l, l}(r) Y_{l, \mathbf{m}}(\Omega) .
$$

Here $f_{n, l}(r)$ is some hypergeometric function [25], whose precise form will not be important for us. The key point is that both the bulk and the boundary fields have the same quantum

\footnotetext{
${ }^{9}$ The fact that the Klein-Gordon equation usually describes bosonic particles does not pose a problem, because we can implement Fermi statistics by hand and take the degeneracy of the spin degrees of freedom into account afterwards. Again, this is very analogous to the standard treatment of the electron spin in atomic physics and is a reliable approximation in the limit of large number of particles.
} 
numbers. This makes the correspondence straightforward. To be precise, the relationship with the operator $\Psi$ in the CFT may be expressed by

$$
\Psi(t, \Omega)|v a c\rangle=\lim _{r \rightarrow \infty} e^{\Delta_{0} r / \ell} \psi(t, r, \Omega)|v a c\rangle
$$

This implies that the creation modes that we defined in the CFT are indeed directly related to those constructed out of the bulk fields. The Hilbert spaces are therefore completely identified, at least in this free field limit. This also implies that the composite operator that we constructed indeed may be identified with a multiparticle state, whose complete wave function may be represented in the bulk as a Slater determinant in terms of the modes given in (2.16).

$$
|\mathbf{\Phi}\rangle=\prod_{n, l, m} \alpha_{n, l, m}^{\dagger}|v a c\rangle
$$

\section{$2.3 \quad H y d r o d y n a m i c ~ d e s c r i p t i o n$}

In this section we will give a hydrodynamic description of the multi-particle state as a free degenerate Fermi gas in AdS space. The Fermi gas is found to be confined to a spherical region with a radius determined by the ratio of the Fermi momentum and the fermion mass. This fact is due to the gravitational potential well of the AdS space and not due to self-gravity.

\subsubsection{The equation of state}

At first one may think that the equation of state for a degenerate Fermi gas in AdS space is different from that in flat spacetime. For instance, the spacing of the lowest energy levels are determined by the AdS radius, and differ from that of flat space. But when the number of particles within one AdS volume is very large, one can safely ignore the effect of the curvature radius, and use a local description of the gas as if it lived in flat spacetime. As we will see this approximation reproduces the exact results of the previous subsection in the limit of large number of particles.

To make this point more clear, consider a degenerate free Fermi gas in a box of size $L$ in $d$ spatial dimensions. The Fermi momentum $k_{F}$ is related to the chemical potential $\mu$ via

$$
\mu=\sqrt{k_{F}^{2}+m_{f}^{2}} .
$$

At zero temperature all states satisfying the bound

$$
\frac{2 \pi|\vec{n}|}{L} \leq k_{F}
$$

are being occupied. Semi-classically we can count states by calculating the volume of phase space. Hence, the particle number density $\mathbf{n}$ is equal to the volume of the d-dimensional Fermi sphere:

$$
\mathbf{n}=\frac{V_{d-1} k_{F}^{d}}{d(2 \pi)^{d}} .
$$

Here $V_{d-1}$ is the area of a unit $(d-1)$-sphere. 
These are the usual relations that hold in flat space. But as long as the number of particles per AdS volume is very large, the Fermi momentum will be much larger than the inverse AdS radius $\ell$. Therefore, in order to determine the equation of state one can take the size $L$ of the box very small compared to $\ell$. This means that the flat space relations given above also apply locally in the curved AdS background. The only difference with a translationally invariant flat space situation is that in this case one should expect that the Fermi momentum $k_{F}$ and chemical potential $\mu$ depend on the position in the AdS space.

The energy density and pressure are given by the standard expressions

$$
\begin{aligned}
& \rho=\frac{V_{d-1}}{(2 \pi)^{d}} \int_{0}^{k_{F}} d k k^{d-1} \sqrt{k^{2}+m_{f}^{2}}, \\
& p=\frac{V_{d-1}}{d(2 \pi)^{d}} \int_{0}^{k_{F}} d k \frac{k^{d+1}}{\sqrt{k^{2}+m_{f}^{2}}},
\end{aligned}
$$

and obey the standard thermodynamic relations

$$
d \rho=\mu d \mathbf{n}, \quad \rho+p=\mu \mathbf{n} .
$$

One small comment about the energy density $\rho$ will be useful. Namely, it can be written as

$$
\rho=\int_{m_{f}}^{\epsilon_{F}} \epsilon \frac{d \mathbf{n}}{d \epsilon} d \epsilon
$$

Intuitively, the validity of this identity should be obvious: one simply adds all energies $\epsilon$ with a weight given by the local density of particles with that energy. Similarly the pressure can be written as

$$
p=\int_{m_{f}}^{\epsilon_{F}} \mathbf{n}(\epsilon) d \epsilon
$$

\subsubsection{Energy momentum conservation}

In the hydrodynamic limit we expect to be able to describe the free Fermi gas purely in terms of the local energy density $\rho$, the pressure $p$, and a local velocity field $u^{\mu}$ normalized so that $u^{\mu} u_{\mu}=-1$. The energy momentum tensor is given in terms of these quantities as

$$
T_{\mu \nu}=(\rho+p) u_{\mu} u_{\nu}+p g_{\mu \nu}
$$

We will first consider the non-rotating degenerate state and postpone the discussion of the rotating state to section 2.4. Without rotation the energy density and pressure will only depend on the radius $r$. In this section we are still ignoring the effect of self-gravity of the gas. However, we still have to take into account the effect of the curved AdS background. This is done by imposing conservation of the energy momentum tensor. For a static configuration it is the equivalent of imposing hydrostatic equilibrium. Anticipating possible generalizations let us write the metric in the general form

$$
d s^{2}=-A(r)^{2} d t^{2}+B(r)^{2} d r^{2}+r^{2} d \Omega_{d-1}^{2} .
$$


The Fermi gas is static, and hence we take $u_{t}=A(r)$ and all other components of $u$ equal to zero. The only non-trivial equation is $\nabla^{\mu} T_{\mu r}=0$, which becomes

$$
\frac{d p}{d r}+\frac{A^{\prime}}{A}(\rho+p)=0
$$

This equation is surprisingly easy to solve. By making use of the identities (2.23) one easily verifies that (2.27) is satisfied when the chemical potential obeys

$$
\mu(r)=\frac{\epsilon_{F}}{A(r)},
$$

where at this stage $\epsilon_{F}$ is an arbitrary constant. We conclude that the radial dependence of the chemical potential is simply due to the gravitational redshift. With some hindsight this result could have been anticipated: it is an analogue of the familiar Tolman relation for the temperature, which is known to hold for any static gravitational field.

So far, this discussion has been general in the sense that we have not used the explicit form of the AdS metric. But let us now go back to the specific case of the AdS space time, and investigate its energy and pressure profile in more detail. For the AdS metric (2.14) the relation (2.28) becomes

$$
\mu(r)=\frac{\epsilon_{F}}{\sqrt{1+r^{2} / \ell^{2}}} .
$$

Now that we have determined the radial profile $\mu(r)$ of the chemical potential we in principle also know the value of the energy density $\rho(r)$ and the pressure $p(r)$ through the relations (2.22). It is clear from this result that the chemical potential decreases as one goes out to the boundary. It can not decrease arbitrarily, however, since the energy per particle is always larger than the mass $m_{f}$. Namely, when $\mu=m_{f}$ the Fermi momentum $k_{F}$ vanishes and so do the energy density and pressure. We conclude therefore that $\rho(r)$ and $p(r)$ will go to zero at a finite value $R$ of the radius, namely when

$$
\mu(R)=m_{f}
$$

It will be interesting to re-express the value of the radius $R$ in terms of the Fermi momentum $k_{F}(0)$. Combining the equations (2.29) and (2.30) together with the standard relation between $k_{F}$ and $\epsilon_{F}$ gives

$$
R=\frac{k_{F}(0)}{m_{f}} \ell
$$

Hence, the size of the star in AdS units is a direct measure for how relativistic the fastest particles are in its center. When the size of the star is of the order of the AdS radius $\ell$ these particles are mildly relativistic, while they have ultra-relativistic velocities when the star is much larger than $\ell$. It is important to note that the Fermi gas has a sharp edge only when the fermions are massive. For massless fermions the equation of state is simply that of a relativistic gas, and the star will spread out with a dilute "tail" towards the boundary of AdS. ${ }^{10}$

\footnotetext{
${ }^{10}$ Of course when the density gets too low the hydrodynamic approximation breaks down.
} 


\subsubsection{Total mass and particle number}

The degenerate star-like object that we described in the previous subsections is the holographic dual of the boundary state $|\Phi\rangle$ constructed in section 2.1. As a non-trivial check on our hydrodynamic description one can verify that the particle number and mass agree with the boundary calculations. We observe that the constant $\epsilon_{F}$ equals the chemical potential defined with respect to the time $t$. This means that it can be identified with the Fermi energy $\epsilon_{F}$ of the CFT

$$
\epsilon_{F}^{C F T}=\epsilon_{F}^{\mathrm{AdS}}
$$

The total particle number in the bulk is given by the integral

$$
N_{F}^{\text {AdS }}=V_{d-1} \int_{0}^{R} d r r^{d-1} B(r) \mathbf{n}(r) .
$$

The factor $B(r)$ comes from the volume form on the spatial section. Similarly, one obtains the mass $M^{\text {AdS }}$ by integrating the energy density. Because $M^{\text {AdS }}$ is defined with respect to the time coordinate $t$ one has to include a redshift factor $A(r)$. For the AdS metric (2.14) the redshift factor $A(r)$ cancels the measure factor $B(r)$, and hence

$$
M^{\mathrm{AdS}}=V_{d-1} \int_{0}^{R} d r r^{d-1} \rho(r)
$$

To calculate $N_{F}^{\text {AdS }}$ and $M^{\text {AdS }}$ one has to insert the equations (2.21) and (2.22) for the particle and energy density and include the redshift effect (2.28) into the chemical potential. The resulting integrals can be performed analytically and (perhaps not surprisingly) precisely reproduce the results (2.12) and (2.13) in the limit of large number of particles.

\subsubsection{A comment on the validity of the hydrodynamic description}

In general, a basic assumption of the hydrodynamic approximation is that the continuous system under investigation is in local thermodynamic equilibrium. More precisely the hydrodynamic description is an expansion in (space-time) derivatives of local thermodynamic quantities. As such, hydrodynamics is valid when the typical variation length is much larger than the microscopic scales of the fluid. In particular it should be much larger than the mean free path. In our case the "fluid" is actually a free gas, so the mean free path is infinite or at most bounded by the AdS scale. This means that strictly speaking we are not allowed to use hydrodynamics. In particular if we had considered time dependent configurations, then there would clearly be a problem since our fermions are non-interacting and there would be no restoring agent to bring them back to equilibrium. ${ }^{11}$ However for our static, spherically symmetric situation the results derived by the hydrodynamic method are reliable. This is indicated by the agreement of (2.10), (2.11) with (2.33), (2.34) and could perhaps be made more quantitative along the lines of [26-28].

\footnotetext{
${ }^{11}$ Once we consider $1 / N$ corrections the particles will become interacting and they will of course reach equilibrium.
} 


\subsubsection{Radial reconstruction}

In the limit of a large number of fermions the hydrodynamic description in the bulk is more convenient than keeping track of the exact quantum state (2.18). We saw in sections 2.1 and 2.2 that at the level of multiparticle quantum states we have a one-to-one correspondence between the boundary and the bulk. This means that we should be able to construct the analogue of the "hydrodynamic description" in the boundary CFT. In other words, we would like to understand what is the CFT meaning of a hydrodynamic description of the multitrace operator $(2.2)$.

The hydrodynamic description of the gas in the bulk is partly encoded in the radial density profile $\rho(r)$ given by $(2.22)$ and $(2.28)$. How is $\rho(r)$ related to the operator $(2.2)$ ? The radial direction $r$ does not have a direct meaning in the CFT, though in a sense it corresponds to the "scale" of the excitation. One way to probe the scale direction is to consider 2-point functions of operators in the CFT, evaluated on the state (2.2). The separation of the two insertions of operators on the boundary controls how deeply in the bulk we are probing: when the two operators are close to each other we are probing the region near the boundary, while at larger separations of the boundary operators we probe the interior and in particular the region where the fermionic gas lives. Hence if we compute the 2-point function

$$
\langle\overline{\mathbf{\Phi}}|\bar{\Psi}(x) \Psi(y)| \mathbf{\Phi}\rangle
$$

in the limit where the number of particles in $|\Phi\rangle$ is large, we should be able to relate the radial profile $\rho(r)$ to the $(x-y)$ dependence of this correlator.

Computing this correlator should be straightforward in the free $c \rightarrow \infty$ limit and it would be interesting to perform this computation, as this would be the first step before considering the same problem including backreaction. We hope to revisit this question in future work.

\subsubsection{Fermions at finite temperature}

It is not difficult to generalize the story to the case where the fermions are at non-zero temperature. Let us assume that the fermions are in an ensemble characterized by the average occupation number $\xi(\epsilon)$ for the one-particle states which are multiplied together to make the analogue of the multi-trace operator (2.2). ${ }^{12}$ In the zero temperature case the occupation numbers are given by the zero temperature Fermi-Dirac distribution $\xi(\epsilon)=$ $\Theta\left(\epsilon_{F}-\epsilon\right)$, where $\Theta$ is the unit-step function. This distribution corresponds to the radial profile (2.29) of the local chemical potential in the bulk. We denote the corresponding density and pressure profiles by $\rho_{\epsilon_{F}}(r), p_{\epsilon_{F}}(r)$.

What happens if we consider a more general distribution $\xi(\epsilon)$ for the average occupation level of the single-trace operators? Notice that formally we can write any function $\xi(\epsilon)$

\footnotetext{
${ }^{12}$ For simplicity we assume that we are in an ensemble where the average occupation level $\xi(\epsilon)$ depends only on the energy of the state. This assumption guarantees that the configuration will be spherically symmetric in the bulk.
} 
defined for $\epsilon \geq 0$ as a linear combination of $\Theta$-functions in the following way

$$
\xi(\epsilon)=-\int_{0}^{\infty} d \epsilon_{F} \xi^{\prime}\left(\epsilon_{F}\right) \Theta\left(\epsilon_{F}-\epsilon\right)
$$

which can be proven by a partial integration, assuming that $\xi$ vanishes at $\epsilon_{F}=\infty$. Since the fermions are non-interacting this implies that the bulk profile can be written as

$$
\rho_{\xi}(r)=-\int_{0}^{\infty} d \epsilon_{F} \xi^{\prime}\left(\epsilon_{F}\right) \rho_{\epsilon_{F}}(r) \quad, \quad p_{\xi}(r)=-\int_{0}^{\infty} d \epsilon_{F} \xi^{\prime}\left(\epsilon_{F}\right) p_{\epsilon_{F}}(r)
$$

In particular for a thermal distribution in the grand-canonical ensemble at temperature $\beta$ and chemical potential $\mu$ we have

$$
\xi(\epsilon)=\frac{1}{e^{\beta(\epsilon-\mu)}+1}
$$

Using (2.29), (2.36) and (2.37) we can compute the radial profile of the density (and pressure) corresponding to a finite temperature fermion gas in AdS, before we consider any backreaction. Let us notice that while the equations (2.36) seem to work for any choice of $\xi$, the profile in the bulk will have a meaningful description in terms of a density profile $\rho_{\xi}(r)$ only if $\xi$ is sufficiently smooth.

It would be interesting to compute the entropy of a thermal ensemble from the fluid configuration in the bulk. For large quantum numbers this entropy should agree with the exact boundary computation of the entropy in terms of the quantum states of the single-trace operators.

Finally, from the relation (2.36) it should be clear that the mapping between perturbations of the bulk density profile and the boundary is non-local, as expected from the general framework of AdS/CFT.

\subsection{Rotating star}

As a straightforward generalization and as a further test of the hydrodynamic approximation we will now try to incorporate rotation into the system. For simplicity we focus on $\mathrm{AdS}_{5}$ and we take the metric to be

$$
d s^{2}=-\left(1+\frac{r^{2}}{\ell^{2}}\right) d t^{2}+\left(1+\frac{r^{2}}{\ell^{2}}\right)^{-1} d r^{2}+r^{2}\left(d \theta^{2}+\sin ^{2} \theta d \phi^{2}+\cos ^{2} \theta d \psi^{2}\right) .
$$

The range of the variable $\theta$ is $[0, \pi / 2]$. In order to put angular momentum into the system we need to take a rotating fluid, and there is an obvious question whether or not the rotation is uniform. In four dimensional flat space it is claimed in [29, 30] that uniform rotation minimizes the mass of the star. We are going to try to do the same thing for the five-dimensional AdS case.

The energy momentum tensor still equals

$$
T_{\mu \nu}=(\rho+p) u_{\mu} u_{\nu}+p g_{\mu \nu}
$$

where now $p$ and $\rho$ will be functions of both $r$ and $\theta$, and

$$
u^{\mu}=U^{-1}\left(1,0,0, \Omega_{\phi}, \Omega_{\psi}\right)
$$


where

$$
U=\left(\left(1+\frac{r^{2}}{\ell^{2}}\right)-r^{2} \sin ^{2} \theta \Omega_{\phi}^{2}-r^{2} \cos ^{2} \theta \Omega_{\psi}^{2}\right)^{1 / 2} .
$$

Conservation of $T$ implies

$$
\begin{aligned}
& 0=U^{2} \partial_{r} p+\frac{r}{\ell^{2}}(p+\rho)\left(1-\ell^{2} \Omega_{\psi}^{2} \cos ^{2} \theta-\ell^{2} \Omega_{\phi}^{2} \sin ^{2} \theta\right) \\
& 0=U^{2} \partial_{\theta} p+r^{2}(p+\rho)\left(\Omega_{\psi}^{2}-\Omega_{\phi}^{2}\right) \sin \theta \cos \theta
\end{aligned}
$$

These equations are very simple, they are equivalent to

$$
\begin{aligned}
& 0=\partial_{r} p+\frac{\partial_{r} U}{U}(p+\rho) \\
& 0=\partial_{\theta} p+\frac{\partial_{\theta} U}{U}(p+\rho) .
\end{aligned}
$$

We can now immediately solve for the star, the solution simply reads

$$
\mu(r)=\frac{\epsilon_{F}}{\mathrm{U}(r)}
$$

If $\Omega_{\phi}, \Omega_{\psi}$ depend non-trivially on $r, \theta$ this simple solution is no longer correct.

Given the exact solution, we wish to write expressions for the mass and two angular momenta. These are all related to Killing vectors $\xi^{\mu}$ and the conserved charges boil down to

$$
Q[\xi]=4 \pi^{2} \int \xi^{\mu} T_{\mu 0}\left(1+\frac{r^{2}}{\ell^{2}}\right)^{-1} r^{3} \sin \theta \cos \theta d r d \theta
$$

In particular, we obtain for the mass

$$
M=4 \pi^{2} \int r^{3} \sin \theta \cos \theta d r d \theta\left(-p+\frac{1+r^{2} / \ell^{2}}{U^{2}}(p+\rho)\right)
$$

and for the angular momenta

$$
J_{\phi}=-4 \pi^{2} \int r^{3} \sin \theta \cos \theta d r d \theta \frac{\Omega_{\phi} r^{2} \sin ^{2} \theta}{U^{2}}(p+\rho) .
$$

and

$$
J_{\psi}=-4 \pi^{2} \int r^{3} \sin \theta \cos \theta d r d \theta \frac{\Omega_{\psi} r^{2} \cos ^{2} \theta}{U^{2}}(p+\rho) .
$$

These are rather difficult expressions, which simplify quite a bit if we put

$$
\Omega_{\phi}=\Omega_{\psi}=\frac{\omega}{\ell} .
$$

One can then explicitly do the relevant integrals, and we obtain

$$
M=\frac{\ell^{4}}{120} \frac{\left(\epsilon_{F}-m_{f}\right)^{4}\left(4 \epsilon_{F}+m_{f}-5 m_{f} \omega^{2}\right)}{\left(1-\omega^{2}\right)^{3}}
$$

and

$$
J=-\frac{\ell^{6}\left(\epsilon_{F}-m_{f}\right)^{5} \omega}{60\left(1-\omega^{2}\right)^{3}} .
$$


Let us now describe the rotating degenerate state in the boundary CFT. For this purpose we choose a $\mathrm{U}(1)$ subgroup in one of the two $\mathrm{SU}(2)$ factors. To be specific, let us consider a rotation associated with, say, a simultaneous right handed rotation in $\phi_{1}$ and $\phi_{2}$. The corresponding quantum number is

$$
j=m_{1}+m_{2} .
$$

Single particle states are characterized by their angular momentum $l$ and energy $E_{0}=$ $\Delta_{0}+n$. When $n$ is fixed but $l$ is left free $j$ takes the following values

$$
-n \leq j \leq n,
$$

but remains even (odd) when $n$ is even (odd).

We describe the rotating degenerate state by introducing an angular velocity $\omega$ which acts as a "chemical potential" for the quantum number $j$. This means that for a given Fermi energy $\epsilon_{F}$ all states are being occupied which obey the inequality

$$
n+\Delta_{0}-\omega j \leq \epsilon_{F} \ell .
$$

Thus the effect of the rotation is to tilt the Fermi surface to favor positive values of $j$.

To count the total number of particles contained in this rotating degenerate many particle state we have to add all one particle states labeled by $n$ and $j$ that satisfy the inequality (2.54). As a preparation, let us count how many single particle states exist for given values of $n$ and $j$. This is most easily done by making use of a generating function for the symmetrized tensor products of the vector representation of $\mathrm{SO}(4)$. One can easily convince oneself that this generating function is given by

$$
\frac{1}{(1-q z)^{2}\left(1-q z^{-1}\right)^{2}}
$$

Here $q$ keeps track of $n$, while $z$ keeps track of the $j$ quantum number. Picking the term proportional to $q^{n} z^{j}$ gives the following degeneracy

$$
\mathcal{N}(n, j)=\frac{1}{4}\left((n+2)^{2}-j^{2}\right)
$$

Note that for even (or odd) $n$ the highest number of states occurs at $j=0$ (or $j= \pm 1$ ). For $j= \pm n$ one finds precisely one state, as expected.

This result can now be used to count the number of particles in the rotating degenerate state for a given chemical potential $\epsilon_{F}$. We will do so in the limit of many particles, so that we can replace the summation over the quantum numbers $n$ and $j$ by integrals. In fact, for this purpose it is convenient to replace $n+2$ simply by $n$. This leads to the following expression

$$
N\left(\epsilon_{F}, \omega\right)=\frac{1}{2} \int_{\mathcal{D}_{\epsilon_{F}, \omega}} \mathcal{N}(n, j) d n d j .
$$

where $\mathcal{D}_{\epsilon_{F}, \omega}$ denotes the integration domain bounded by (2.54), (2.53) and $n \geq 0$. The factor of $1 / 2$ is due to the fact that the values of $j$ are only even or odd. The integrals are straightforward but somewhat tedious. After some work we find

$$
N\left(\epsilon_{F}, \omega\right)=\frac{\ell^{4}}{24} \frac{\left(\epsilon_{F}-m_{0}\right)^{4}}{\left(1-\omega^{2}\right)^{2}}
$$


We see that for a given chemical potential the number of particles increases due to the rotation. Note further that in the limit $\omega \rightarrow \pm 1$ the number of particles diverges. This can be understood from the fact that in this case all states with $j= \pm n$ are being counted for arbitrary values of $n$.

The mass and angular momentum can be obtained in a similar way as integrals over $n$ and $j$ over the domain $\mathcal{D}_{\epsilon_{F}, \omega}$. For the mass one has to sum up the energy $\epsilon=\left(n+\Delta_{0}\right) / \ell$ for all particles contained in the rotating degenerate state. This leads to the integral expression

$$
M=\frac{1}{2 \ell} \int_{\mathcal{D}_{\epsilon_{F}, \omega}}\left(n+\Delta_{0}\right) \mathcal{N}(n, j) d n d j .
$$

Performing this integral is again straightforward. The final result can be organized in the following form

$$
M=\frac{\ell^{4}}{30} \frac{\left(\epsilon_{F}-m_{0}\right)^{5}}{\left(1-\omega^{2}\right)^{3}}+\frac{m_{0} \ell^{4}}{24} \frac{\left(\epsilon_{F}-m_{0}\right)^{4}}{\left(1-\omega^{2}\right)^{2}} .
$$

This result should be compared to the case without rotation given in (2.11). The last term represents the contribution of the mass $m_{0}$ for all the particles, whose number is given in (2.58), while the first term gives the sum of all the $n$ quantum numbers. We note that the modification in the mass due to the rotation is again given in terms of inverse powers of $\left(1-\omega^{2}\right)$, and diverges for $\omega \rightarrow \pm 1$.

Finally, let us come to the angular momentum $J$. The total angular momentum carried by the particles in the rotating degenerate state is represented by again a tedious but fortunately straightforward integral

$$
J=\frac{1}{4 \ell} \int_{\mathcal{D}_{\epsilon_{F}, \omega}} j \mathcal{N}(n, j) d n d j .
$$

It yields the following result

$$
J=\frac{\ell^{4}}{60} \frac{\left(\epsilon_{F}-m\right)^{5}}{\left(1-\omega^{2}\right)^{3}} \omega
$$

Note that $|J|<M$ in general, and only when $\omega \rightarrow \pm 1$ the ratio $|J| / M \rightarrow \pm 1$.

The results (2.60), (2.62) for the mass and angular momentum computed in the conformal field theory agree with the hydrodynamic computations (2.50), (2.51).

\section{$3 \quad$ Including interactions}

\subsection{The scaling limit}

The discussions of the previous section are accurate if we take the $c \rightarrow \infty$ limit keeping $\Delta$, $N_{F}$ and $\Delta_{0}$ fixed. In that limit the system is free as interactions can be ignored due to 't Hooft factorization. However what we have constructed so far is a trapped fermionic gas rather than a star. To turn the gas into a star we have to include gravitational backreaction. This can be achieved by scaling $\Delta_{0}$ and $N_{F}$ (and as a result $\Delta$ ) appropriately at the same time that we send $c$ to infinity.

To determine what is the precise scaling limit we should take, we consider the picture in the bulk. The first condition we impose is that the radius of the star is of the order of the 
AdS scale $\ell .^{13}$ Assuming that equation (2.31) is a good estimate for the order of magnitude of the size of the star even after backreaction, ${ }^{14}$ we see that this condition implies

$$
\frac{k_{F}}{m_{f}}=\frac{n_{F}}{\Delta_{0}}=\text { fixed }
$$

The second condition is that we want to have appreciable gravitational backreaction. This can be translated to the statement $G M \ell^{2-d} \sim \mathcal{O}(1)$. Newton's constant is related to the central charge by $G \sim c^{-1} \ell^{d-1}$. So we find that the conformal dimension of the composite operator must satisfy

$$
\frac{\Delta}{c}=\text { fixed }
$$

where we used the identification $\Delta=M \ell$. From equation (2.4) and (3.1) we find that for this to be true we have to take

$$
n_{F}, \Delta_{0} \sim c^{\frac{1}{d+1}}
$$

In this limit the number of particles (2.3) goes like

$$
N_{F} \sim c^{\frac{d}{d+1}}
$$

In other words we want to study a specific class of composite multitrace operators in the limit $c \rightarrow \infty, \Delta=\varepsilon c$ where $\varepsilon$ is kept fixed. When $\varepsilon \ll 1$ the system is weakly interacting. We want to turn on $\varepsilon$ up to values of order 1 where interactions become important. ${ }^{15}$

Since in this limit the number of particles becomes large, it is conceivable that the 't Hooft factorization might fail in a drastic way. In terms of Feynman diagrams in a gauge theory this can happen because we are scaling the number of external lines of a diagram with $N$ so the usual $N$-counting rules may not apply. Let us describe the problem in some more detail: consider the correlation functions of multitrace operators whose size we keep fixed in the large $N$ limit. As far as interactions between distinct single trace constituents are concerned, the dominant diagrams are the disconnected ones, which correspond to the free limit discussed in section 2. Connected planar diagrams involving interactions between various single trace components of the multitrace operator are suppressed by powers of $\frac{1}{N}$ relative to the disconnected ones and non-planar diagrams by even higher powers of $\frac{1}{N}$. Now let us imagine scaling the size of the multitrace operators as we send $N \rightarrow \infty$. In order to have nontrivial interactions at large $N$ we want to scale the size of the multitrace operators in such a way as to compensate for the $\frac{1}{N}$ suppression of connected planar diagrams (connecting the various single trace components of the multi-trace operators). Our goal is to work in a limit where these leading connected planar diagrams are of the same order as the disconnected ones. The danger now is that since in this limit the planar connected diagrams became as important as the disconnected ones, then the same could happen for non-planar diagrams. This would imply that effects of quantum-gravity would

\footnotetext{
${ }^{13}$ By this we mean that as $c \rightarrow \infty$ the radius of the star scales like as $R \sim c^{0} \ell$.

${ }^{14}$ This assumption will be proven to be self-consistent after we actually compute the backreaction.

${ }^{15}$ Let us note that even though in our limit the particles become very heavy (since their rest mass scales like $\left.\ell^{-1} c^{\frac{1}{d+1}}\right)$, they are parametrically lighter than the Planck mass which scales like $\ell^{-1} c^{\frac{1}{d-1}}$.
} 
become important in the bulk. However we will argue below that this is not the case and the planar diagrams remain the important ones.

It is perhaps more intuitive to address this question from the bulk point of view. In theories with a gravitational dual the planar interactions on the boundary are mapped to tree level supergravity diagrams in the bulk. The $c \rightarrow \infty$ limit corresponds to sending $G \rightarrow 0$ in AdS units. In this limit the tree level interaction between any two particles (whose mass is kept fixed) will vanish. In our double scaling limit we are taking the number and masses of particles to be large so that even though $G \rightarrow 0$ the total backreaction remains finite. Moreover the various factors contribute in such a way that the backreaction receives contributions from tree level diagrams only, as we explain in the next subsection. This implies that the planar approximation remains reliable in this limit.

\subsection{Interacting quantum states in AdS}

Let us now consider the many-body state (2.18) in the scaling limit of the previous subsection, in which we have to take into account the gravitational interactions between the fermions. It is intuitively clear that in the limit of large number of fermions per unit volume, the most efficient way to analyze the interactions is by working in the hydrodynamic description of section 2.3, but this time incorporating the gravitational backreaction of the fermionic fluid. This approach will be pursued in subsection 3.4. For now we will consider the interactions from a more microscopic point of view, in order to understand the validity of approximations involved in going from the exact to the coarse grained hydrodynamic description.

A first simplification is that in our scaling limit (and for theories with a semi-classical gravity dual) we can ignore processes which change the number of fermions. In a relativistic system the number of particles does not have to be conserved. If we put a certain number of fermions in AdS and then turn on interactions it is not guaranteed that their number will remain constant. Unless the fermions are protected by a conservation law, there will be processes which can change their number, as well as processes which produce all kinds of other particles such as gravitons etc. In principle all of these have to be taken into account. However in our scaling limit the fermionic gas becomes effectively long lived i.e. the rate of creation and annihilation of particles becomes negligible. This is due to the fact that at large $c$ interactions are suppressed. In principle this suppression could be compensated by large combinatoric factors due to the large number of particles, but as we will discuss in more detail in section 6 , these factors work out in such a way that the processes which change the particle number become subleading at large $c$, relative to processes responsible for the total gravitational backreaction of the fermions. Because of this, in the rest of this section we will only focus on interactions which do not change the number of particles, i.e. on processes with only $N_{F}$ incoming and $N_{F}$ outgoing fermions and no other external particles.

Second, we will assume that the only low lying fields in the bulk are the graviton, described by the Einstein-Hilbert action, minimally coupled to a fermionic field $\psi$ of mass 


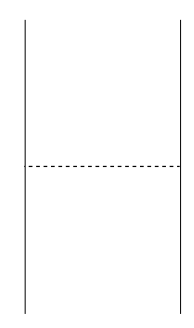

Figure 1. Tree level gravitational interaction between two fermions.

$m_{f} .{ }^{16}$ We will assume that there are no other types of particles or forces in the bulk. Clearly this is a very drastic approximation which however captures the qualitative behavior of the phenomena we want to study without unnecessary complications. It would be very interesting to study further whether a specific AdS/CFT setup can be found where such a simple bulk Lagrangian can be realized (at low energies), or at least a setup in which it correctly captures the qualitative physics of degenerate fermionic states. We present some first discussions along these lines in section 6 .

We now want to see that in our scaling limit the $\frac{1}{c}$ suppression of interactions combines with the enhancement from the large number of particles in such a way that only the tree level interactions (or equivalently planar interactions) become important. To illustrate this point we focus on one particular physical observable as an example: we will try to estimate the total energy of the fermions including their gravitational interactions. So let us consider a bulk state $|\Phi\rangle$ corresponding to $N_{F}$ fermions in the free theory. It satisfies

$$
H_{0}|\Phi\rangle=\frac{\Delta_{0}}{\ell}|\Phi\rangle
$$

where $H_{0}$ is the Hamiltonian for a free fermionic field in $\mathrm{AdS}_{d+1}$. When we include interactions we have to use the full Hamiltonian $H=H_{0}+H_{\text {int }}$ where $H_{\text {int }}$ includes the coupling between fermions and gravitons and the self-interactions of the gravitons and $H_{0}$ has to be extended to include the quadratic part of the Einstein-Hilbert action for propagating gravitons. The corrected energy $\Delta / \ell$ can be computed by an expansion in Feynman diagrams in the bulk.

For example, consider two fermions in AdS with energies $E_{1}, E_{2}$ and wavefunctions $\psi_{1}(x), \psi_{2}(x)$. To leading order the total energy is the sum of the energies. To compute the first correction to this energy we have to compute the tree level gravitational interaction between them. In the Born approximation this is given by the diagram shown in figure 1, where we have to use the graviton and fermion propagators in $\mathrm{AdS}_{d+1}$. In our scaling limit this diagram is proportional to

$$
G \frac{m_{1} m_{2}}{\ell^{d-2}} \sim c^{-\frac{d-1}{d+1}}
$$

\footnotetext{
${ }^{16}$ There may be interaction terms for the field $\psi$, but if their strength is controlled by $c$ in the standard way (i.e. a vertex with $n$ fermions is suppressed by a factor of $c^{\frac{2-n}{2}}$ ) then the qualitative results of this section remain the same. For simplicity we will ignore such terms in what follows.
} 


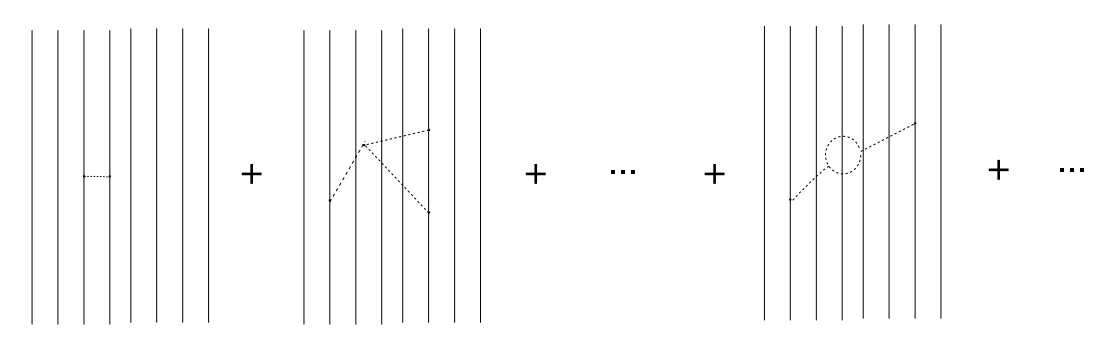

Figure 2. 2-body, 3-body etc. interactions at tree level and higher loop interactions.

so it goes to zero. Higher order diagrams between two fermions are suppressed by further powers of $\frac{1}{c}$, so they all become negligible in the large $c$ limit. Hence if we only had a finite number of particles the backreaction would be unimportant.

If we have $N_{F}$ fermions then to compute the correction to the energy (or the conformal dimension of the dual operator) $\delta \Delta=\Delta-\Delta_{0}$ we have to sum over many kinds of diagrams as shown in figure $2,{ }^{17}$ where as we explained before we only consider diagrams with the same number of fermions in the past and future infinity. Due to the non-linearities of general relativity we not only have 2-particle interactions but also n-particle ones for all $\mathrm{n}$ as depicted in the diagram. We have to sum up all these diagrams to get the right answer. The correction to the energy of the state can be schematically written as

$$
\delta \Delta=\sum_{k=2, L=0}^{\infty} \delta \Delta_{k}^{L}
$$

where $\delta \Delta_{k}^{L}$ denotes the contribution of diagrams where $k$ fermions are involved in the interaction and $L$ is the number of loops.

We will now see how diagrams of different types scale in our limit. Going back to the single graviton exchange between a pair of fermions, but now summing over all possible pairs, we find that the correction to the energy is

$$
\delta \Delta_{2}^{0} \sim \sum_{i \neq j} G \frac{m_{i} m_{j}}{\ell^{d-2}} \sim \frac{c}{\ell}
$$

where we used that $N_{F} \sim c^{\frac{d}{d+1}}$ and $m \sim c^{\frac{1}{d+1}}$. In other words

$$
\frac{\delta \Delta_{2}^{0}}{\Delta_{0}} \sim \mathcal{O}(1)
$$

so the correction is of the same order as the original energy of the system $\Delta_{0}$ and remains important even in the large $c$ limit. The suppression that we found earlier has been compensated by the large number of pairs that we have to sum over. On the other hand, loop diagrams between pairs of fermions are suppressed by additional power of $\frac{1}{c}$ and are unimportant in our scaling limit.

\footnotetext{
${ }^{17}$ Only connected diagrams have to be considered for the computation of $\Delta$. The disconnected diagrams can be resummed into the exponential in $e^{-i t\left(H_{0}+H_{\mathrm{int}}\right)}|\Phi\rangle=e^{-i(\Delta / \ell) t}|\Phi\rangle$.
} 
For a general diagram with $L$ loops, $k$ fermions involved in the interaction (i.e. $k$ ingoing and $k$ outgoing ones), $V_{F}$ fermion-fermion-graviton vertices, and $V_{n}$ vertices of $n$-graviton type, we have the following topological constraints

$$
V_{F}+\sum n V_{n}=2 I_{g} \quad, \quad 2 V_{F}=2 I_{F}+2 k \quad, \quad L=I_{F}+I_{g}-V_{F}-\sum V_{n}+1
$$

where $I_{g}, I_{F}$ denote the number of internal graviton and fermion lines. Now let us count powers of $c$ of these diagrams. Each external line contributes a factor of $\left(N_{F} m_{f}\right)$ which in our scaling limit is proportional to $c$. Each n-graviton vertex contributes a power of $c^{1-\frac{n}{2}}$ and each of the $V_{F}$ vertices a factor of $c^{-\frac{1}{2}}$. So in total the powers for a diagram are

$$
\delta \Delta_{k}^{L} \sim c^{k+\sum\left(1-\frac{n}{2}\right) V_{n}-\frac{V_{F}}{2}}
$$

Using the three conditions that we found above this becomes

$$
\frac{\delta \Delta_{k}^{L}}{\Delta_{0}} \sim c^{-L}
$$

So we find that that only $L=0$ (tree level) diagrams contribute significantly at large $c$. The conclusion is the following: in our double scaling limit we have contributions from diagrams of all orders due to the non-linear nature of general relativity, but only tree level ones. In this sense our system becomes (semi)-classical, though nonlinear.

\subsection{From quantum states to fermionic fluid}

Clearly summing up all such diagrams between fermions is not the most efficient way to analyze the problem and an approximation method would be desirable. In this section we review some basic ideas which are often used in treatments of many-body systems and which may clarify the conceptual steps in going from the quantum many-particle description in the bulk to the hydrodynamic description and the TOV equations of the next subsection. The reader who is not interested in these issues can skip this subsection and go directly to section 3.4.

In general, in a many-body quantum problem we first want to determine the ground state wavefunction and energy, and then perhaps to study small excitations around it. The simplest example to consider is $N_{F}$ non-interacting fermions moving in an external potential. In this case we first compute the energy levels $\epsilon_{i}$ for a single fermion in this potential and the corresponding wavefunctions $\psi_{i}(x)$. The ground state of the manyfermion system is then described simply by filling up these orbitals consistently with the Pauli exclusion principle. The wavefunction is a Slater determinant of the one-particle wave functions

$$
\Psi_{N_{F}}\left(x_{1}, \ldots, x_{N}\right)=\frac{1}{\sqrt{N_{F} !}}\left|\begin{array}{cccc}
\psi_{1}\left(x_{1}\right) & \psi_{2}\left(x_{1}\right) & \ldots & \psi_{N_{F}}\left(x_{1}\right) \\
\psi_{1}\left(x_{2}\right) & \psi_{2}\left(x_{2}\right) & \ldots & \psi_{N_{F}}\left(x_{2}\right) \\
& \ldots & \\
\psi_{1}\left(x_{N_{F}}\right) & \psi_{2}\left(x_{N_{F}}\right) & \ldots & \psi_{N_{F}}\left(x_{N_{F}}\right)
\end{array}\right|
$$


and the total energy is given by the sum of the individual energies

$$
E_{\mathrm{tot}}=\sum_{i=1}^{N_{F}} \epsilon_{i}
$$

When we turn on interactions between the fermions the situation is not as simple, since it is no longer true that the many-body state can be constructed from the knowledge of single particle energy states. More precisely, single particle energy states, or "orbitals" are not even well defined in an interacting system of fermions. By adding or removing a fermion we affect the wavefunctions of all other fermions in a complicated way, which in turn influences the available energy levels for the fermion under consideration. Hence in principle we have to solve the coupled problem of determining the wavefunction for all fermions at once. This also means that the ground state cannot necessarily be written as a Slater determinant. ${ }^{18}$

A typical problem of this type is finding the ground state of a polyelectronic atom. That problem is quite similar to finding the ground state of our fermionic star, where the role of the attractive Coulomb force from the nucleus is played by the AdS gravitational potential and the electromagnetic interactions of the electrons correspond to the gravitational force between the fermions (though the latter is attractive). As we know, solving Schroedinger's equation for a polyelectronic atom is not possible analytically and we have to address the problem using various approximation methods. We have to do the same for our system.

In our system the interaction between any two particular fermions is negligible in the large $c$ limit due to the $\frac{1}{c}$ suppression. However since the number of fermions is also very large the effect of all of the other fermions on a single fermion can be appreciable. This type of many-body problem can usually be treated by mean field theory methods. We replace all interactions on a given fermion by an effective external field, which has to be determined in a self-consistent way, as we explain below.

\subsubsection{The Hartree-Fock approximation}

In many-body problems the Hartree-Fock approximation is often employed. Its basic assumption is that the ground state of the many-fermion system can be written as a single Slater determinant $|\Psi\rangle$ of one-fermion orbitals in some appropriately chosen external potential, which is determined in a self-consistent manner. One then minimizes the norm of $\langle\Psi|H| \Psi\rangle$ with respect to the single-particle orbitals, where $H$ is the full Hamiltonian. This leads to the Hartree-Fock equations for the single-particle orbitals $\psi_{i}$ and the corresponding energies $\epsilon_{i}$.

In general the physical meaning of the single-particle energies $\epsilon_{i}$ in the Hartree-Fock approximation is not transparent: for example if we try to add one more fermion to a previously unoccupied state $\epsilon_{j}$, then the energy of the new state will not be exactly equal to $E_{\text {tot }}+\epsilon_{j}$. This is due to the fact that when we add a fermion all other fermions will rearrange themselves and in principle one has to solve the Hartree-Fock problem again for $N_{F}+1$ fermions, which will introduce corrections to the energy, additional to the naive

\footnotetext{
${ }^{18}$ However it can be written as a superposition of Slater determinants.
} 
estimate $E_{\text {tot }}+\epsilon_{j}$. For the same reason exciting a fermion from the state $\epsilon_{i}$ to the state $\epsilon_{j}$ will cost energy equal to $\epsilon_{j}-\epsilon_{i}$ plus corrections due to rearrangement.

Nevertheless in the limit of large number of fermions the aforementioned corrections due to rearrangement become negligible, at least for certain many-body systems. This is sometimes called "Koopmans theorem", which basically states that in the limit of large number of particles we can treat any given one of them as a "probe" and define its energy as if it had negligible backreaction on the rest of the particles. Hence in this limit the excitation energies of a single fermion are simply determined by the Hartree-Fock spectrum $\epsilon_{i}$, by taking the difference of the energy of the final minus the initial state. Similarly if we think of constructing the ground state by gradually adding fermions and rearranging them to their lowest available energy state, then $\epsilon_{F}$ would be the energy of the last added fermion. Notice that these statements hold only if we talk about changes to a few numbers of fermions relative to the total number of them.

Within the context of the Hartree-Fock approximation we can define a notion of single particle density of states, even for a system of interacting fermions

$$
g\left(\epsilon, \epsilon_{F}\right) \equiv \frac{d N}{d \epsilon}\left(\epsilon, \epsilon_{F}\right)
$$

which is now a function of the "Fermi energy" $\epsilon_{F}$. This function encodes the density of the single-particle wavefunctions of the Hartree-Fock solution with corresponding energies $\epsilon_{i}$. We call Fermi energy $\epsilon_{F}$ the highest energy of the occupied states and $N\left(\epsilon, \epsilon_{F}\right)$ is the number of occupied single-particle states with energy lower than or equal to $\epsilon$. Clearly we have $N_{F}=N\left(\epsilon_{F}, \epsilon_{F}\right)$. In the limit of large number of particles, where Koopmans theorem or the probe approximation holds, the function $g\left(\epsilon, \epsilon_{F}\right)$ can be determined operationally by doing "spectroscopy" on the state, as follows: we consider the system in its ground state. We hit the state by a photon coupled to the fermions and measure the absorption spectrum. This gives information about the energy differences between the single fermion states (assuming that Koopmans theorem holds) and in this way we can reconstruct the function $g\left(\epsilon, \epsilon_{F}\right)$.

The total number of fermions can be written as

$$
N_{F}\left(\epsilon_{F}\right)=\int_{\epsilon_{\min }}^{\epsilon_{F}} d \epsilon g\left(\epsilon, \epsilon_{F}\right)
$$

where $\epsilon_{\min }$ is the lowest of the single-particle states. ${ }^{19}$ But now it is important that the total energy of the system $E_{\text {tot }} \neq \int_{\epsilon_{\min }}^{\epsilon_{F}} d \epsilon \epsilon g\left(\epsilon, \epsilon_{F}\right)$. This is due to the interactions between the fermions and the rearrangement issues which cannot be ignored if one tries to build up the ground state by adding fermions gradually, since this process involves all of the fermions and clearly goes beyond the "probe" limit. However this can still be done for the last added fermion, so the following is still true

$$
\frac{d E_{\mathrm{tot}}}{d \epsilon_{F}}=\epsilon_{F} g\left(\epsilon_{F}, \epsilon_{F}\right)
$$

\footnotetext{
${ }^{19}$ Notice that due to the interactions the energy $\epsilon_{\min }$ can be low, for example lower than the rest mass of the fermions.
} 
It is clear that for our problem, that is, for determining the ground state of a large number of gravitationally interacting fermions in AdS, the Hartree-Fock method is applicable and moreover the assumptions of Koopmans theorem are satisfied. This means that the Hartree-Fock orbitals should be really thought as the possible quantum states of a fermion moving in the gravitational background produced by rest of the fermions and the density of states (3.3) describes the distribution of fermions with various energies, generalizing the non-interacting density of state (2.13).

In practice we will not apply the Hartree-Fock method, since it is still too complicated when the number of fermions is large. As we explain below, a further approximation in addition to the Hartree-Fock can be made, which leads naturally to the TOV equations.

\subsubsection{Fermion fluid and Thomas-Fermi approximation}

Even after imposing the Hartree-Fock approximation we can make a further simplification as follows: instead of solving for the ground state Slater-determinant wavefunction, we can only look for the fermion number density $\mathbf{n}(x)$ in the ground state ${ }^{20}$

$$
\mathbf{n}(x)=\int d^{d} x_{2} \ldots d^{d} x_{N}\left|\Psi\left(x, x_{2}, \ldots, x_{N_{F}}\right)\right|^{2}
$$

which satisfies $\int d^{d} x \mathbf{n}(x)=N_{F}$. Of course $\mathbf{n}(x)$ carries less information than the actual wavefunction but it is easier to determine than $\Psi$. We express the kinetic and potential energy of the fermions in terms of $\mathbf{n}(x) .{ }^{21}$ Minimizing the energy with respect to $\mathbf{n}(x)$ determines the fermion density profile and the energy of the ground state. This is the Thomas-Fermi approximation. In many systems the Thomas-Fermi approximation becomes asymptotically exact in the limit of large number of particles. As we will explain later, the hydrodynamic approximation and the TOV equations in the bulk is the equivalent of the Thomas-Fermi approximation (or rather its relativistic generalization) for the quantum interacting fermions of the previous sections, also see references [26-28].

\subsection{TOV equations in the bulk}

After these general comments we return to what we would have naively done to deal with the gravitational backreaction of a large number of fermions: we revisit our hydrodynamic formalism of section 2.3, but now taking into account the gravitational backreaction of the fermionic fluid. We will explain how the hydrodynamic approximation is related to the Hartree-Fock and Thomas-Fermi approximations in the next subsection

We have to solve Einstein's equations with cosmological constant coupled to matter

$$
R_{\mu \nu}-\frac{1}{2} g_{\mu \nu} R+\Lambda g_{\mu \nu}=8 \pi G T_{\mu \nu}
$$

\footnotetext{
${ }^{20}$ In this section we think of fermions moving in flat $d$-dimensional space. If we are in curved spacetime then the appropriate measure factors have to be included.

${ }^{21}$ For example for a system of many (non-relativistic) electrons moving in an external potential $V(x)$ the total energy in the Thomas-Fermi approximation would be

$$
E_{T F}(n)=K \int d^{3} x \mathbf{n}(x)^{5 / 3}+\int d^{3} x V(x) \mathbf{n}(x)+\frac{e^{2}}{2} \iint d^{3} x d^{3} y \frac{\mathbf{n}(x) \mathbf{n}(y)}{|x-y|}
$$
}


where the source is a perfect fluid

$$
T_{\mu \nu}=(\rho+p) u_{\mu} u_{\nu}+p g_{\mu \nu}
$$

and $\rho, p$ are determined by the flat space equation of state (2.22) for a degenerate fermionic fluid.

As before we look for static, spherically symmetric solutions, so we write the metric in the general form

$$
d s^{2}=-A(r)^{2} d t^{2}+B(r)^{2} d r^{2}+r^{2} d \Omega_{d-1}^{2} .
$$

We parametrize the functions $A(r)$ and $B(r)$ in terms of two new functions $M(r)$ and $\chi(r)$ as

$$
\begin{aligned}
& A^{2}(r)=e^{2 \chi(r)}\left(1-\frac{C_{d} M(r)}{r^{d-2}}+\frac{r^{2}}{\ell^{2}}\right), \\
& B^{2}(r)=\left(1-\frac{C_{d} M(r)}{r^{d-2}}+\frac{r^{2}}{\ell^{2}}\right)^{-1},
\end{aligned}
$$

where Newton's constant is contained in the coefficient

$$
C_{d}=\frac{16 \pi G}{(d-1) V_{d-1}}
$$

The Fermi gas is static, and hence we take $u_{t}=A(r)$ and all other components of $u_{\mu}$ equal to zero. In terms of $M(r)$ and $\chi(r)$ the Einstein equations read

$$
\begin{aligned}
\frac{d M}{d r} & =V_{d-1} r^{d-1} \rho \\
\frac{d \chi}{d r} & =V_{d-1} \frac{C_{d}}{2}(\rho+p) r B^{2} \\
\frac{d p}{d r}+\frac{A^{\prime}}{A}(\rho+p) & =0
\end{aligned}
$$

These constitute the Tolman-Oppenheimer-Volkoff (TOV) equations.

We choose as boundary condition $M(0)=0$, so that $M(r)$ represents the contribution to the mass from the energy density inside a ball of radius $r$, and the total mass is equal to $M(R)$. By Birkhoff's theorem the metric outside the star is given by AdSSchwarzschild. Hence,

$$
\chi(r)=0, \quad M(r)=M, \quad \text { for } r \geq R .
$$

From (2.30) it follows that the radius $R$ is determined by

$$
1-\frac{C_{d} M}{R^{d-2}}+\frac{R^{2}}{\ell^{2}}=\left(\frac{\epsilon_{F}}{m}\right)^{2} .
$$

Finally the total fermion number can be computed by the integral

$$
N_{F}=V_{d-1} \int_{0}^{R} d r r^{d-1} B(r) \mathbf{n}(r)
$$


Let us now see how we can compute the density of single-particle states $g\left(\epsilon, \epsilon_{F}\right)$ from the hydrodynamic configuration in the bulk. The energy $\epsilon$ of a fermion should be identified with the redshifted energy of a fermionic particle in the bulk. At a given point in the bulk we have fermions with various values of the local energy ranging from the fermion mass $m$ up to the local Fermi energy $\mu(r)$. So we can write the following formula for the density of states on the boundary

$$
\begin{aligned}
N\left(\epsilon, \epsilon_{F}\right) & =V_{d-1} \int_{0}^{R} d r B(r) r^{d-1} \mathbf{n}\left(\frac{\epsilon}{A(r)}\right) \\
g\left(\epsilon, \epsilon_{F}\right) & =\frac{d N}{d \epsilon}\left(\epsilon, \epsilon_{F}\right)
\end{aligned}
$$

where $\mathbf{n}(\epsilon)$ was defined in (2.21). Notice that the statement that $E_{\text {tot }} \neq \int d \epsilon \epsilon g\left(\epsilon, \epsilon_{F}\right)$ is related to the statement in the bulk that the total mass is not equal to the sum of the redshifted masses of the particles.

In fact, to show that the relation (3.5) is still valid one needs to use the Einstein equations! One has

$$
\begin{aligned}
\epsilon_{F} \frac{d N}{d \epsilon_{F}} & =V_{d-1} \int_{0}^{R} d r r^{d-1}\left(B \epsilon_{F} \frac{d \mathbf{n}}{d \epsilon_{F}}+\frac{d B}{d \epsilon_{F}} \epsilon_{F} \mathbf{n}\right) \\
& =V_{d-1} \int_{0}^{R} d r r^{d-1} e^{\chi}\left(\frac{d \rho}{d \epsilon_{F}}+\frac{d B}{d \epsilon_{F}} \frac{\rho+p}{B}\right) \\
& =\int_{0}^{R} d r e^{\chi}\left(\frac{d M^{\prime}}{d \epsilon_{F}}+\frac{d M}{d \epsilon_{F}} \chi^{\prime}\right)=\frac{d M}{d \epsilon_{F}} .
\end{aligned}
$$

Here we used the identity (2.23), the relation (2.28), the TOV equations (3.9), the definitions of $A$ and $B$ in terms of the functions $M$ and $\chi$ and the fact that at the edge of the star $\mathbf{n}(R)=0$. Notice that above the local particle number density depends on the radius as $\mathbf{n}(r)=\mathbf{n}\left(\epsilon_{F} / A(r)\right)$.

In the next section we will use the TOV equations to calculate the corrections to the mass, the particle number and the density of states defined through the relations (2.12).

\subsection{Relation between quantum description and TOV approach}

What is the precise relation between the analysis in terms of quantum states and Feynman diagrams in section 3.2 and the hydrodynamic TOV equations (3.9)? The Einstein equations coupled to a perfect fluid are in a certain sense the mean field/Thomas-Fermi approximation for a system of fermions interacting gravitationally. This correspondence is of course implicitly used in the standard treatment of astrophysical neutron stars, in which the star is treated as a classical self-gravitating ball of fluid governed by a fermionic equation of state and not as a quantum bound state of individual fermions. The logic in going from the quantum bound state to the fluid description was outlined in subsection 3.3.

The equivalence between these two approaches can be made more precise. In $[26,27]$ the following problem was analyzed: the authors considered the Schroedinger equation for $N_{F}$ fermions mutually interacting with a Newtonian attractive gravitational potential. They passed to the Thomas-Fermi description ${ }^{22}$ replacing the ground state wavefunction

\footnotetext{
${ }^{22}$ With a relativistic kinetic term.
} 
by the fermion density $n(x)$. They then showed that in the ground state the fermion density satisfies the (non-relativistic limit of) the TOV equations. In general relativity one should also include higher order forces in the Schroedinger equation. Presumably the Thomas-Fermi approximation would then reproduce the full relativistic TOV equations. ${ }^{23}$

In [28] a related analysis was followed: the starting point is a static spherically symmetric metric parametrized by two arbitrary functions $A(r), B(r)$ as in (3.7). One solves the Dirac equation for fermions moving in this background and places $N_{F}$ of them in the lowest available states. Then one computes the stress energy tensor produced by these fermions and uses it as a source for Einstein equations for the metric (3.7). Then one shows that in the limit of large $N_{F}$ solving this problem self-consistently is equivalent to solving Einstein equations coupled to a perfect fermionic fluid, that is the TOV equations (3.9).

The reason that we mention all these issues is the following: as explained above, in the bulk we have an intuitive understanding of how to start with the microscopic many-fermion quantum description and truncate it to the Thomas-Fermi type approximation of the TOV equations coupled to a fermionic fluid. What is the equivalent procedure on the boundary? In the boundary CFT the description in terms of individual quantum states for the fermions is easier to understand, at least in the $c \rightarrow \infty$ limit before taking the backreaction into account. It would be very interesting to perform the equivalent "coarse graining" on the boundary and to introduce the boundary analogue of the density of fermions (3.6) and the TOV equations. The main obstacle in this direction is understanding how to deal with the "radial direction" in boundary language, see also the discussions in subsection 2.3.5. We hope to revisit these issues in future work.

\subsection{Quantum states vs. classical geometries in AdS/CFT}

Before we close this section we would like to explain an important conceptual point. In AdS/CFT we have an equivalence of two quantum systems, which means that their Hilbert spaces are isomorphic. In particular every quantum state on the boundary should be dual to a quantum state in the bulk. On the gravity side and at low energies, the Hilbert space can be approximated by the Fock space generated by the semiclassical quantization of the supergravity fields. At infinite $N$ these fields become free, so we simply have to canonically quantize free fields around an AdS background. This semiclassical quantization leads to a Fock space which is isomorphic to the low-conformal dimension Hilbert space of the boundary theory, as we discussed in detail in the previous subsections.

Our many-particle state is a quantum state: it is a many-particle state constructed by acting on the vacuum with many creation operators of the semi-classically quantized free fields in AdS. Hence it should not be confused with a classical configuration in the bulk, i.e. a classical supergravity solution. The latter should be thought of as a coherent state where certain modes of the bulk fields have been coherently excited. In this sense our star differs from the "boson stars" (see [31] and references therein) and other solitonic configurations in the bulk. Instead, a bosonic analogue of our fermionic star would be, for

\footnotetext{
${ }^{23}$ However we should mention that one qualitative feature of the solution, the existence of a critical mass - the Chandrasekhar bound, is also visible in the Newtonian gravity approximation.
} 
example, a finite temperature bosonic gas like the radiation star considered by Page and Phillips in [32] or the "geons" of Wheeler [33].

Let us explain this in some more detail: the large $N$ limit is usually thought of as a classical limit. Of course no matter how large $N$ is, if the energy of the state under consideration is low enough then one would still see the quantization and the discreteness of the spectrum. Let us now consider classical supergravity solutions in the bulk. Such solutions have energy of order $N^{2}$ as can be seen by the overall normalization of the bulk action. However the opposite is not true: a state whose energy is of order $N^{2}$ (at large $N$ ) does not necessarily correspond to a classical supergravity solution. Our quantum states are examples of this kind. The difference between the two types of states (coherent vs multi-particle quantum states) is that while the total energy is the same (order $N^{2}$ ), the distribution of the energy to various modes is different: in the coherent states we have few modes excited many times, while in our quantum states we have many modes excited few times. ${ }^{24}$ The latter cannot be described by a classical field. More generally, it is important to remember that the fermionic perfect fluid is not a "classical field" in the bulk, even thought it is described by a classical density profile.

Perhaps a useful analogy to keep in mind is the difference between a classical electric field and black body radiation. The former has non-vanishing energy density at the classical level. By tuning the temperature of the black body radiation appropriately as $\hbar \rightarrow 0$ we can end up with a finite classical energy density for the radiation. However there is no sense in which the black body radiation can be described by a classical electromagnetic field. The reason is that the energy is distributed in too many modes unlike what happens in a coherent state.

\section{Numerical solutions and gravitational collapse}

\subsection{Numerical results}

We will now look for static, spherically symmetric solutions of the TOV equations (3.9). These equations cannot be solved analytically and we have to resort to numerical integration. We present the numerical analysis for $\mathrm{AdS}_{5}$, i.e. $d=4$. The qualitative features of our results are the same for other values of $d$. As we explained in section 3.1 we are working in the limit $G_{5} \ell^{-3} \rightarrow 0, M \ell \rightarrow \infty$, or in CFT language $c \rightarrow \infty, \Delta \rightarrow \infty$. It is more convenient to present the graphs in terms of rescaled dimensionless quantities which stay finite in this limit. For the total mass and the total fermion number we define

$$
\hat{M} \equiv \frac{\Delta}{c}=\frac{8}{\pi} \frac{G_{5}}{\ell^{2}} M \quad, \quad \hat{N}_{F} \equiv \frac{N_{F}}{c^{4 / 5}}
$$

and for the fermion mass and local chemical potential in the bulk

$$
\hat{m}_{f} \equiv \frac{\Delta_{0}}{c^{1 / 5}}=\left(\frac{8}{\pi} \frac{G_{5}}{\ell^{3}}\right)^{1 / 5}\left(m_{f} \ell\right) \quad, \quad \hat{\mu} \equiv\left(\frac{8}{\pi} \frac{G_{5}}{\ell^{3}}\right)^{1 / 5}(\mu \ell)
$$

All hatted quantities are kept fixed as $c=\frac{\pi \ell^{3}}{8 G_{5}} \rightarrow \infty$.

\footnotetext{
${ }^{24}$ We are grateful to S. Minwalla for discussions on these points.
} 


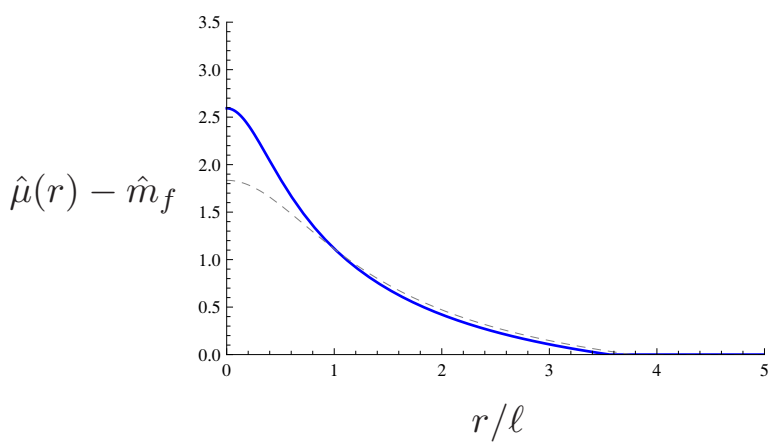

Figure 3. Radial profile of the local chemical potential with (solid line) and without gravitational backreaction (dashed line).

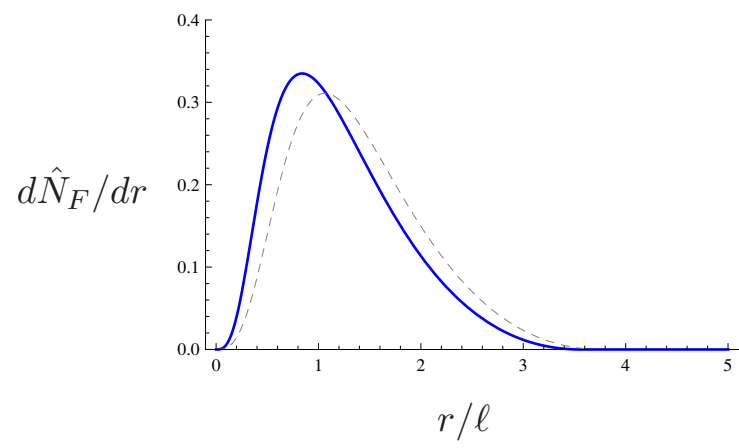

Figure 4. Fermion number density, with and without backreaction.

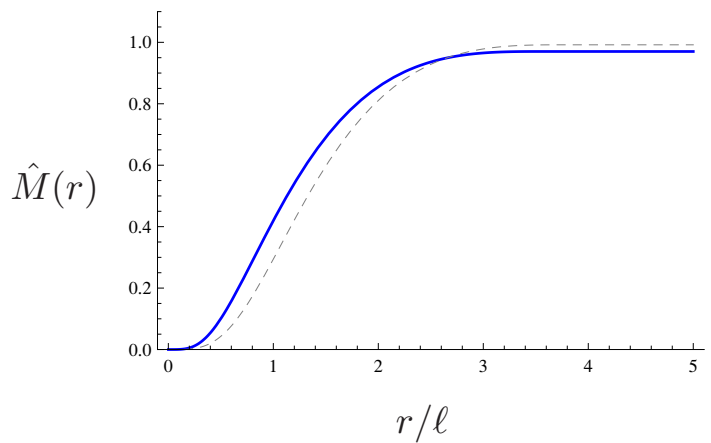

Figure 5. Mass function with and without backreaction. The limiting value as $r / \ell \rightarrow \infty$ is the total mass $M$ of the system.

\subsubsection{A typical fermionic star}

We first describe a star of typical values for the total number of fermions $\hat{N}_{F}$ and fermion mass $\hat{m}_{f}$. We plot the radial profile of the local chemical potential in the bulk and the fermion number density in figures 3 and 4 respectively. The dotted lines in the same graphs show what would have been the profiles for the same number of fermions in AdS without gravitational backreaction. The edge of the star is the point where (2.30) is satisfied and the density goes to zero. We can see from the graphs that turning on self-gravity moves the fermions towards the center of AdS, as expected.

In figure 5 we plot the function $\hat{M}(r)$ introduced in (3.8), which asymptotes to the total mass of the solution as $r / \ell \rightarrow \infty$. We see that turning on gravitational interactions 


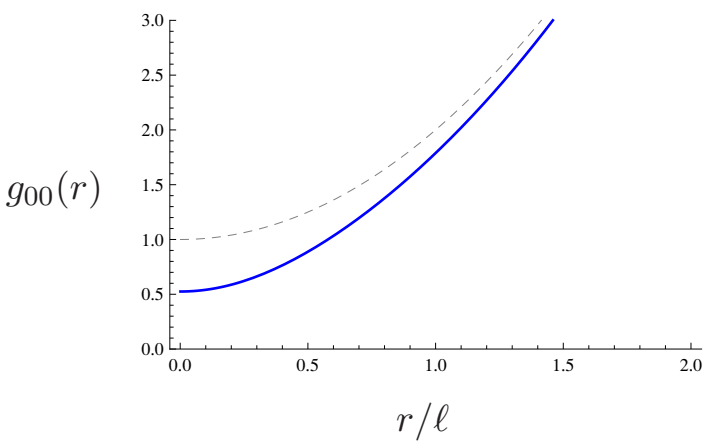

Figure 6. Gravitational potential of the star (solid) vs that of empty AdS (dashed).

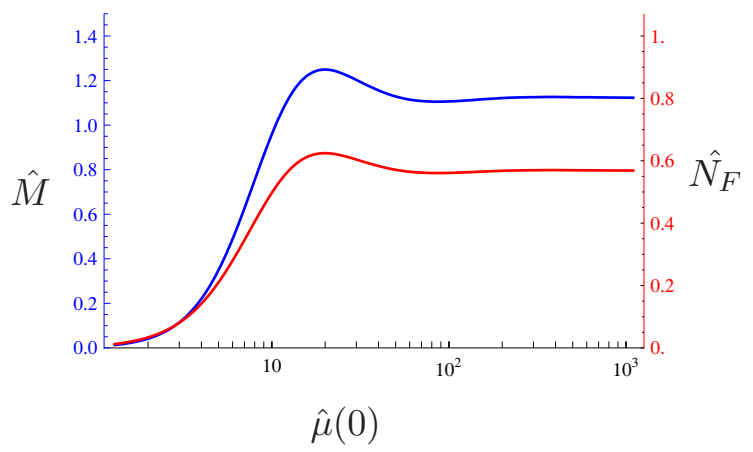

Figure 7. Total mass (blue) and fermion number (red) vs chemical potential at the center of the star.

lowers the total mass. The difference between the two curves can be understood as the binding energy. Remarkably, we see that the binding energy is a very small percentage of the total mass. This fact is not a peculiarity of the solution for the the particular choice of $\hat{N}_{F}, \hat{m}_{F}$ but is generally true for all of their possible values. In figure 6 we show the $g_{00}$ component of the metric in our solution relative to that of empty AdS where we can see the gravitational well produced by the star.

\subsubsection{Families of stars and a critical mass}

We now want to study a family of stars of increasing fermion number, keeping the fermion mass $\hat{m}_{f}$ fixed. For this we would like to find the self-gravitating solution of $\hat{N}_{F}$ fermions in AdS, as a function of $\hat{N}_{F}$. However when solving the TOV equations, rather than fixing the total fermion number $\hat{N}_{F}$, it is technically more convenient to fix the local chemical potential $\hat{\mu}(0)$ at the center of the star and then integrate the equations outwards. Only after the entire solution has been computed, can the number of fermions be evaluated from (3.11).

Following this procedure we plot in figure 7 the mass and fermion number ${ }^{25}$ as a function of the local chemical potential at the center of the star.

\footnotetext{
${ }^{25}$ Let us mention that while $\hat{N}_{F}$ is of order one in this particular example, it does not mean that we have a small number of fermions, since $\hat{N}_{F}$ is a rescaled variable. The actual number of fermions is $N_{F}=\hat{N}_{F} c^{4 / 5}$ which is large for $c \rightarrow \infty$.
} 


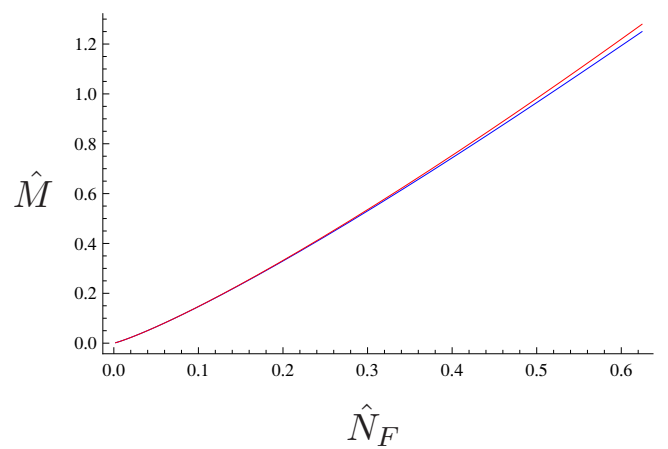

Figure 8. Mass vs Fermion number with (blue) and without (red) backreaction.

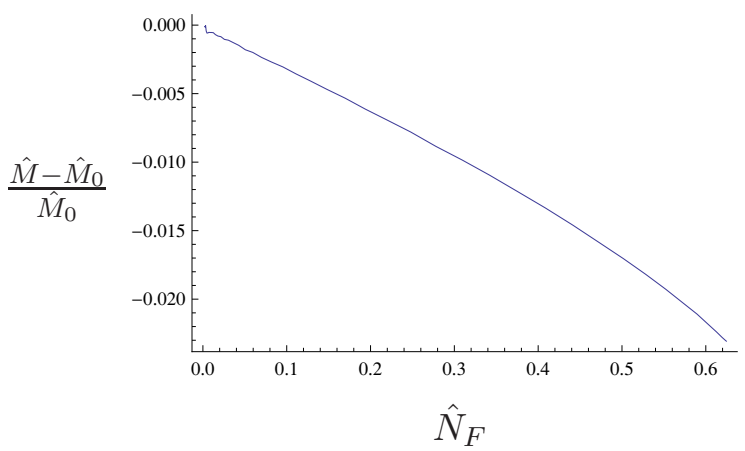

Figure 9. Binding energy as a function of fermion number.

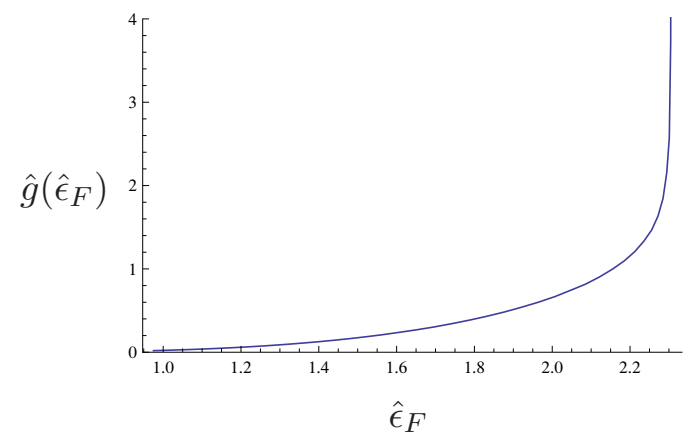

Figure 10. Density of states at the Fermi energy $\hat{\epsilon}_{F}$. The value of $\hat{\epsilon}_{F}$ where the density diverges corresponds via relation (4.1) to the value of $\hat{\mu}(0)$ where the mass reaches its highest value.

The most striking feature of the diagram is the existence of a critical value for the mass and fermion number which is achieved for a certain value $\hat{\mu}_{c}$ of the local chemical potential at the center of the star. This is the Chandrasekhar, or Oppenheimer-Volkoff limit. It will be discussed in more detail in the next subsection but for now there are two important points that we want to emphasize: first, the right way to read figure 7 is that there are no static, spherically symmetric solutions of the TOV equations with a larger number of fermions than that achieved by the solution at $\mu_{c}$. If we insist on starting with initial conditions on a spacelike slice, corresponding to a larger number of fermions, the solution will inevitably become time-dependent and will presumably collapse. Second, as we will explain below the solutions with $\hat{\mu}(0)>\hat{\mu}_{c}$ are unstable under radial perturbations 


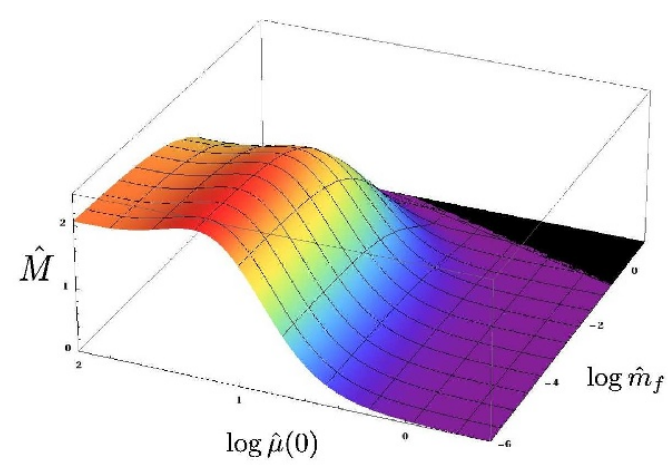

Figure 11. Total mass vs chemical potential and fermion mass.

and hence in a certain sense they should be considered unphysical. We will return to these points in the next subsection.

In figure 8 we show the total mass as a function of the total number of fermions (keeping $\hat{m}_{f}$ fixed). In the same graph we can see what would be the total energy $\hat{M}_{0}$ of the same number of fermions if they were placed in AdS without taking the self-gravitation into account, as computed from (2.10) and (2.11). The difference between the two curves is very small. In figure 9 we see the binding energy relative to $\hat{M}_{0}$. These diagrams reveal a surprising feature of our system: the self-gravitating solution breaks down and becomes unstable at a point where the binding energy is only a few percents of the total energy.

Finally we consider the interpretation of our solution in the Hartree-Fock picture on the boundary. The Fermi energy on the boundary $\epsilon_{F}$ is defined by equation (2.28) so we have

$$
\hat{\epsilon}_{F}=\hat{\mu}(0) \sqrt{g_{00}}(0)
$$

In figure 10 we plot the density of states at the Fermi energy as a function of the Fermi energy $\hat{\epsilon}_{F}$, which can be computed from (3.13). We see that approaching the critical point the density of states blows up, which signifies a break-down of the Hartree-Fock approximation.

\subsubsection{Dependence on the fermion mass}

We now consider how the previous results depend on the fermion mass $\hat{m}_{f}$. In figure 11 we plot the total mass as a function of the fermion mass and the chemical potential. We see that the critical mass and the value $\hat{\mu}_{c}$ at which criticality is achieved depend on the fermion mass $\hat{m}_{f}$. The qualitative behavior is the following: for small fermion mass criticality is achieved for higher values of the chemical potential, where the fermions are in the relativistic regime, and the radius of the star (which we have not plotted) is large relative to the AdS radius. For heavier fermions we have criticality in a regime where the fermions are non-relativistic and the size of the star is smaller than the AdS radius. In figures 12 and 13 we plot the mass and density of states respectively, as a function of the Fermi energy and fermion mass. 


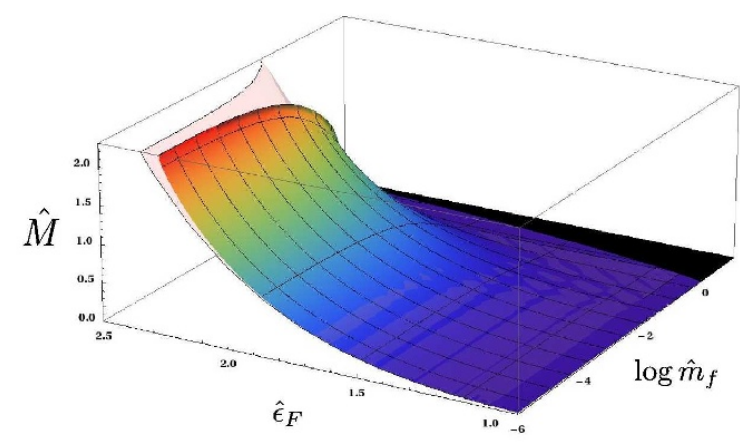

Figure 12. Mass vs Fermi energy and fermion number. The underlying pink graph is the result without self-gravity.

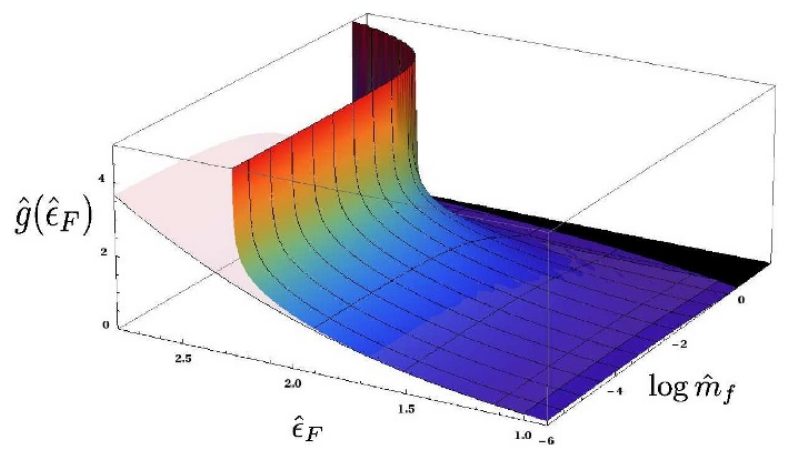

Figure 13. Density of states at the Fermi energy vs Fermi energy and fermion mass. plotted against the graph without backreaction.

\subsection{The Chandrasekhar limit}

The most important qualitative feature of our numerical results is the existence of a maximum value for the mass of a degenerate fermionic star. This value is achieved for a specific critical local chemical potential $\hat{\mu}_{c}$ at the center of the star. It is possible to find static spherically symmetric solutions with higher values of density at the center if we take $\hat{\mu}(0)>\hat{\mu}_{c}$, however it is important to notice that increasing $\hat{\mu}(0)$ above $\hat{\mu}_{c}$ does not correspond to increasing the total number of particles. As we can see from figure 7 the number of particles has a maximum at the same value of $\hat{\mu}(0)$ where the mass reaches its critical value. Solutions with $\hat{\mu}(0)>\hat{\mu}_{c}$ correspond to a different radial distribution of roughly the same number of particles. Moreover as we will argue below solutions with $\hat{\mu}(0)>\hat{\mu}_{c}$ are unstable under radial perturbations.

What happens if we insist on looking for solutions with a larger number of particles? We could do that by starting with initial data on a spacelike hypersurface with a total number of fermions higher than the critical one. Then it is clear that these initial data will evolve into a time-dependent solution describing a collapsing star. This is the point where the degeneracy pressure of the fermions can no longer balance the gravitational attraction, in other words the "Chandrasekhar limit". 
Let us now turn to the stability of solutions with $\hat{\mu}(0)>\hat{\mu}_{c}$. To check the stability we have to linearize the equations of motion around the solution and compute the frequencies of the normal modes $\omega_{n}$, which describe small oscillations around the solution with time dependence of the form $e^{-i \omega_{n} t}$. In general the frequencies are real and the perturbations are simply oscillating with time, while their amplitude remains bounded. If however there is a linearized mode with imaginary frequency, that is with $\omega^{2}<0$, then we see that the perturbation will grow exponentially with time implying that the initial solution was unstable.

While we have not performed the linearized analysis, we summarize a general argument (see for example [34]) which indicates that the solutions with $\hat{\mu}(0)>\hat{\mu}_{c}$ are unstable. Let us say that we have a one-parameter family of static solutions characterized by the central density $\hat{\mu}(0)$. If we have a critical point $\frac{d \hat{M}}{d \hat{\mu}}=0$ at $\hat{\mu}_{c}$, then around that value, to first order, we have two different solutions one with $\hat{\mu}_{c}$ and one with $\hat{\mu}_{c}+\delta \hat{\mu}$ with the same total mass. The change of the profile induced by $\delta \hat{\mu}(0)$ can be thought of as a linearized perturbation of the solution at $\hat{\mu}_{c}$. From the fact that the total mass does not change we conclude that for this perturbation at $\hat{\mu}_{c}$ we have $\omega^{2}=0$, i.e. we have a zero mode. Generically we can assume that $\omega^{2}$ changes sign as we cross $\hat{\mu}_{c}$. If we assume that the solutions for $\hat{\mu}(0)<\hat{\mu}_{c}$ are stable then we conclude that $\omega^{2}<0$ for $\hat{\mu}(0)>\hat{\mu}_{c}$. Hence these solutions will develop a tachyonic mode past the critical point $\hat{\mu}_{c}$. We expect that at the subsequent critical points more modes may become unstable.

\subsection{Endpoint of the collapse}

In a realistic theory the endpoint of the collapse of a degenerate star depends on the details of the particle spectrum, interactions, equation of state etc. For example in the real world a white dwarf can collapse to a neutron star which in turn may collapse to more (hypothetical) exotic states such as quark stars and eventually a black hole. Since we have been mainly concerned with generic features of degenerate stars in AdS we cannot answer this question in detail. However we notice that, at least for light fermions, the critical mass is of the same order as that of a big black hole in AdS. It is reasonable to expect that such an object is the most entropic one, for given total mass. This suggests that the final endpoint of the collapse will be a big black hole in AdS.

Nevertheless we should mention that while the endpoint of the gravitational collapse is very likely a black hole, the onset of the collapse itself does not have to do with black hole physics. The onset of the collapse is marked by the failure of hydrostatic equilibrium for a large number of fermions and can be understood in terms of the low-lying (super)gravity modes.

In principle it would be straightforward to compute numerically the time-dependent solution corresponding to the collapse of a fermionic fluid in AdS towards a black hole. In practice this problem is significantly harder than what we have done so far, since it involves solving partial differential equations instead of ordinary ones (since the fields will now depend on time and radius). We postpone the analysis of the time-dependent solutions and the black hole formation to future work. 


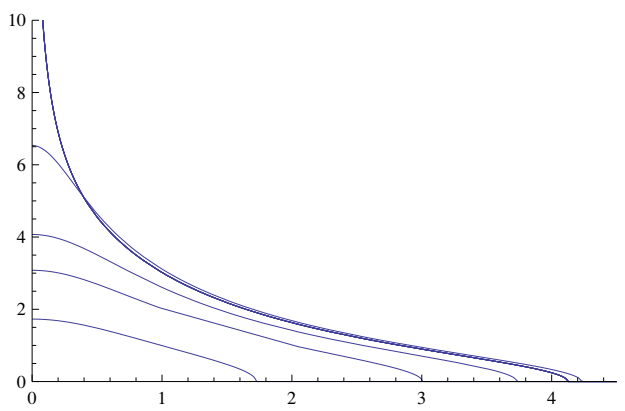

Figure 14. Profile of the local Fermi momentum $\hat{k}_{F}(r)$ for increasing values of $\hat{\mu}(0)$ and the convergence to a limit curve.

\subsection{Scaling regime}

In this section we will explore some features of the solutions deep inside the unstable regime $\hat{\mu}(0) \gg \hat{\mu}_{c}$.

\subsubsection{Limiting solution}

At very large values of the chemical potential at the center one finds that the solution starts to exhibit an interesting behavior: most of the radial profile of the chemical potential approaches a limiting shape, while a sharp "spike" develops at the center of the star. This is shown in figure 14, where we plot the local Fermi momentum as a function of the radius. The Fermi momentum is very large at the center but rapidly drops to the limiting profile. While the spike becomes sharper and sharper as we increase $\hat{\mu}(0)$ the total mass contained in it is finite (the same is true about the number of fermions in the spike), hence the total mass of the solution remains finite.

One might worry that the spike is an artifact of the numerics, but one can actually find an analytic solution which describes its limiting shape. In the limit where $\hat{\mu}(0) \rightarrow \infty$ we can assume that all quantities have a simple scaling behavior as $r \rightarrow 0$. Plugging a power-law ansatz into the TOV equations and looking for a solution in the regime $r \rightarrow 0$ (where the cosmological constant can be neglected) one can fix the scaling solution as

$$
\begin{aligned}
\rho(r) & =\frac{45}{112 \pi G} \frac{1}{r^{2}}+\mathcal{O}(r) \\
B^{2}(r) & =\frac{7}{4}+\mathcal{O}(r) \\
A^{2}(r) & =r^{\frac{1}{5}} b_{3}+\mathcal{O}(r)
\end{aligned}
$$

where the constant $b_{3}$ cannot be fixed by a local analysis. ${ }^{26}$ One can check that the numerical solution of the spike approaches this analytic form in the limit $\hat{\mu}(0) \rightarrow \infty$.

${ }^{26}$ More generally, the scaling solution in $d+1$ bulk dimensions is

$$
\rho(r)=\frac{1}{r^{2}}\left(b_{1}+\mathcal{O}(r)\right), \quad B^{2}(r)=b_{2}+\mathcal{O}(r), \quad A^{2}(r)=r^{\frac{1}{d+1}}\left(b_{3}+\mathcal{O}(r)\right)
$$

where

$$
b_{1}=\frac{1}{4 \pi G} \frac{(d-1)^{2}(d+1)}{(d-1)^{3}+d-3}, \quad b_{2}=\frac{(d-1)^{3}+d-3}{(d-1)^{3}-3 d+1}
$$

and the constant $b_{3}$ is arbitrary. 


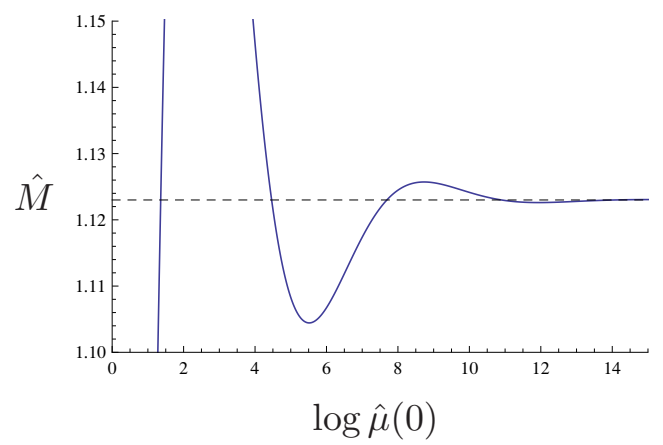

Figure 15. Detail of 7 where we see the damped oscillations around $\hat{M}_{c} \approx 1.1230$. This plot corresponds to $\hat{m}_{f} \approx 0.95$.

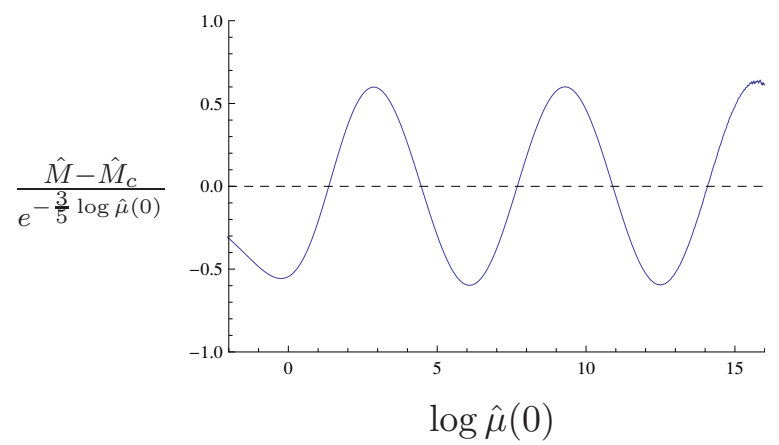

Figure 16. In this plot the oscillations become more visible.

The fact that in the small $r$ limit the TOV equations admit exact scaling solutions in which the central density goes to infinity is a well known fact, see for example [35].

\subsubsection{Oscillations of the mass}

The second interesting feature of the regime $\hat{\mu}(0) \gg \hat{\mu}_{c}$ is the following: ${ }^{27}$ in figure 7 we see that in the limit $\hat{\mu}(0) \rightarrow \infty$ the mass $\hat{M}$ approaches a limiting value $\hat{M}_{c}{ }^{28}$. However we notice that the convergence is not monotonic, but rather the function $\hat{M}(\hat{\mu}(0))$ undergoes small damped oscillations around the critical value $\hat{M}_{c}$. In figure 15 we plot the same function at a different scale, in which the oscillations become more visible. This qualitative behavior appears in many problems of similar kind.

In the limit $\hat{\mu}(0) \rightarrow \infty$ these oscillations can be described by the following formula

$$
\hat{M}(\hat{\mu}) \approx \hat{M}_{c}+A e^{-\gamma \log \hat{\mu}(0)} \cos (\omega \log \hat{\mu}(0)+\delta)
$$

\footnotetext{
${ }^{27}$ This section was added to our paper after similar calculations were carried out in collaboration with S. Bhattacharyya and S. Minwalla in [15]. K.P. would like to thank S. Bhattacharyya and S. Minwalla for very useful discussions on these issues during the collaboration in [15]. We would also like to thank V. Hubeny and M. Rangamani for very useful comments and suggestions regarding the scaling regime and for bringing relevant literature to our attention.

${ }^{28}$ This limiting value depends on the fermion mass $\hat{m}_{f}$.
} 
By fitting to the numerical data we find the following values for these constants ${ }^{29}$

$$
\hat{M}_{c} \approx 1.230, \quad A \approx 0.582, \quad \gamma \approx 0.596, \quad \omega \approx 0.973, \quad \delta \approx 3.508
$$

We will now explain that the formula (4.3) can be justified by analytic methods and the damping constant $\gamma$ and frequency $\omega$ can be determined exactly. Since this aspect is not central to the rest of the paper we will be brief and refer the reader to [15, 36-39] for more details. The main point is to consider the TOV equations as a dynamical system with respect to the variable $r$. In this sense, the critical solution mentioned in the previous subsection acts as an attractor fixed point (in the language of dynamical systems) in the space of solutions of the TOV equations. The critical solution corresponds to $\hat{\mu}(0) \rightarrow \infty$. Solutions with very large but finite $\hat{\mu}(0)$ are in a sense small perturbations of the critical solution and hence their behavior can be understood by analyzing the TOV equations in a linearized approximation around the critical solution. For small $r$ the critical solution is known analytically (4.2) and similarly the eigenvalues of small perturbations around it can be analytically determined by performing a linearized analysis of the TOV equations around the scaling solution. For our system the eigenvalues turn out to be

$$
\lambda=-\frac{6}{5} \pm i \frac{4 \sqrt{6}}{5}
$$

By performing the matching carefully we find that these eigenvalues translate into the following analytic values for the damping constant and frequency of the oscillations of the mass

$$
\gamma=\frac{3}{5} \quad, \quad \omega=\frac{2 \sqrt{6}}{5}
$$

which are in good agreement with the numerical values mentioned above. The small discrepancies are presumably due to various inaccuracies in our numerics.

Before we close this section let us mention that while this oscillatory behavior is clearly interesting, one should keep in mind that it takes place in the regime $\hat{\mu}(0) \gg \hat{\mu}_{c}$ where the solutions are unstable. It would be fascinating if there was a boundary interpretation of the scaling behavior and the oscillations of the mass.

\section{Boundary CFT}

In this section we return to the boundary interpretation of the gravitational interactions between the fermions. As we discussed in section 2 , in the free limit the star is dual to a composite multitrace operator (2.2). If we first fix the size of the operator and then send $c$ to infinity, this correspondence is precise. While the multitrace operator (2.2) is not protected by supersymmetry (i.e. it is not a chiral primary), it is protected due to large $c$ factorization: each of its constituents are (descendants of) chiral primaries and are thus protected. Moreover at infinite $c$ the conformal dimension of composite operators is simply given by the sum of individual dimensions.

\footnotetext{
${ }^{29}$ The precise values of the constants $\hat{M}_{c}, A, \delta$ will in general depend on the value of the fermion mass $\hat{m}_{f}$. The reported values are for $\hat{m}_{f} \approx 0.95$. On the other hand, the constants $\gamma, \omega$ are fixed in an $\hat{m}_{f}$-independent manner and can be determined analytically as explained in the rest of this section.
} 
The interactions between the constituents start to become important in the regime where we scale the (bare) dimension of the operator as $\Delta_{0}=\varepsilon c$ and keep $\varepsilon$ finite. Now we want to understand these interactions from the boundary point of view. We want to work in a regime where the boundary theory has a semiclassical gravity dual, which implies that the gauge coupling must be large (for example large $\lambda \gg 1$ in the $\mathcal{N}=4 \mathrm{SYM}$ ). This means that we cannot analyze the boundary theory perturbatively in the gauge coupling. However even at strong 't Hooft coupling the theory has an expansion in $\frac{1}{c}$. As we will see the $\frac{1}{c}$ expansion together with certain basic assumptions about the boundary CFT can be used to capture some of the qualitative features of the system that we found in section 4 .

In the free theory the conformal dimension of the operator (2.2) is dual to the energy of the fermionic gas in the bulk. The correction to the energy of the gas due to gravitational interactions, that is the binding energy, is related to the anomalous dimensions of operators of the form (2.2), once $\frac{1}{c}$ corrections are taken into account. When $\varepsilon \rightarrow 0$ these corrections become negligible but they are important when $\varepsilon$ is of order 1 . In a certain sense these corrections are controllable as we turn on $\varepsilon$ slowly.

The precise form of the $\frac{1}{c}$ corrections to the composite operators will depend on the details of the conformal field theory. So far we have mainly considered the neutron star in AdS, in a model where the only degrees of freedom consist of a massive fermion coupled to general relativity. Of course, one of our main interests was to determine the dual description of the neutron star and in particular to understand some microscopics of the neutron star instability and its presumed collapse into a black hole. In order to achieve this, we need to embed our discussion in a proper AdS/CFT duality, like e.g. the duality between $\mathcal{N}=4$ SYM theory and type IIB string theory on $\operatorname{AdS}_{5} \times S^{5}$. However, one immediately see that this is potentially difficult. The mass of the fermion $\ell \Delta_{0}$ scales like $N^{2 / 5} \ell$ in the limit we consider, which is larger than the typical mass of an excited string state $1 / \ell_{s} \sim(\lambda)^{1 / 4} \ell$ in the 't Hooft limit. Therefore, one expects that both massive string degrees of freedom, as well as a large number of Kaluza-Klein modes of the massless string degrees of freedom, should be included to properly discuss the physics of the neutron star.

Before discussing these issues in more detail, it is instructive to imagine a situation where these additional degrees of freedom have somehow been decoupled. Though perhaps unrealistic it still interesting to see how much physics such a toy model could capture. In the rest of this section we will focus on a "toy model" CFT, where the only relevant fields are the graviton and the fermion, up to a cutoff of the order of the Planck scale in the bulk.

\subsection{Graviton exchange and first correction to anomalous dimensions}

In the toy model setup the bulk physics is completely described by a massive fermion coupled to general relativity. The corresponding field theory statement is that the only low-lying "single trace operators" in the field theory are the operator $\Psi$ and the stress tensor $T_{\mu \nu}$, together with their conformal descendants. Of course we also have to include their multi-trace composites, as required by crossing symmetry. However these do not correspond to new fields in the bulk, but rather to multi-particle excitations of the basic fields. For simplicity we will restrict attention to the case $d=4$ only. 
In this simple context, it is possible to estimate the point where the instability will appear, using known properties of the operator expansion of conformal field theories in four dimensions. The idea is to consider the anomalous dimension $\Delta$ of the composite operator $\boldsymbol{\Phi}$ as a function of particle number $N_{F}$, and to determine when $d \Delta / d N_{F}$ changes sign, which is the direct analogue of the computation we did in the bulk. Now in order to compute the anomalous dimension of the operator $\boldsymbol{\Phi}$, we are going to use the fact that it is built out of operators $\Psi$ and their derivatives, and the relevant anomalous dimensions can be extracted from correlations functions of $\Psi$ with itself. To compute these correlation functions, we will use the fact that in the OPE of $\Psi$ with itself only the stress-tensor can appear to leading order in $\frac{1}{c}$, apart from multi-particle composites made out of $\Psi$. This simplification may not be accurate in a complete unitary CFT but by assumption this will happen in our toy model. Altogether this computation will therefore be a direct field theory analogue of graviton exchange, where instead of graviton exchange we have the exchange of a stress tensor in the intermediate channel of an OPE.

For future purpose we discuss the relevant computations in a slightly more general setting. Thus, we consider two self-adjoint conformal primaries $O_{1}, O_{2}$. Using the OPE we can define a composite operator : $O_{1} O_{2}$ : whose conformal dimension is $\Delta_{1}+\Delta_{2}$ to leading order in $1 / c$

$$
O_{1}(x) O_{2}(y)=\ldots+: O_{1} O_{2}:(y)+\ldots
$$

This form of the OPE, and the presence of an operator with the quantum numbers and dimension of : $\mathrm{O}_{1} \mathrm{O}_{2}$ : can be extracted by performing a conformal block decomposition of the 4-point function, if we assume that at infinite $c$ it factorizes to a product of 2-point functions.

At the first non-trivial order in $1 / c$ the dimension of the operator : $O_{1} O_{2}:$ will be

$$
\Delta_{12}=\Delta_{1}+\Delta_{2}+\frac{\delta_{12}}{c}
$$

where $\delta_{12}$ is the "anomalous dimension". To extract $\delta_{12}$, we consider the 4-point function

$$
\left\langle O_{1}\left(x_{1}\right) O_{1}\left(x_{2}\right) O_{2}\left(x_{3}\right) O_{2}\left(x_{4}\right)\right\rangle=\frac{1}{\left|x_{12}\right|^{2 \Delta_{1}}\left|x_{34}\right|^{2 \Delta_{2}}}+\mathcal{O}\left(\frac{1}{c}\right)
$$

Here we assumed that the two-point function of $O_{1}$ and $O_{2}$ vanished to leading order in $1 / c$. Now let us see what happens at order $\frac{1}{c}$. As explained above, we will only consider the effect of a stress-tensor in some intermediate channel, e.g. the (12) $\rightarrow$ (34) channel. The appearance of $T$ in the OPE of $O_{1} O_{1}$ is controlled by the Ward identities and we have schematically [40]

$$
O_{1}(x) O_{1}(y) \sim \ldots+\Delta_{1} \frac{T}{|x-y|^{2 \Delta_{1}-4}}+\ldots
$$

while

$$
O_{2}(x) O_{2}(y) \sim \ldots+\Delta_{2} \frac{T}{|x-y|^{2 \Delta_{2}-4}}+\ldots
$$

and also

$$
T(x) T(y) \sim \frac{c}{|x-y|^{8}}
$$




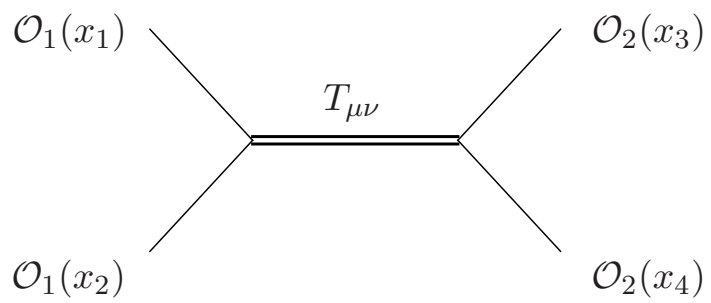

Figure 17. Conformal partial wave corresponding to the exchange of $T_{\mu \nu}$ in the double OPE.

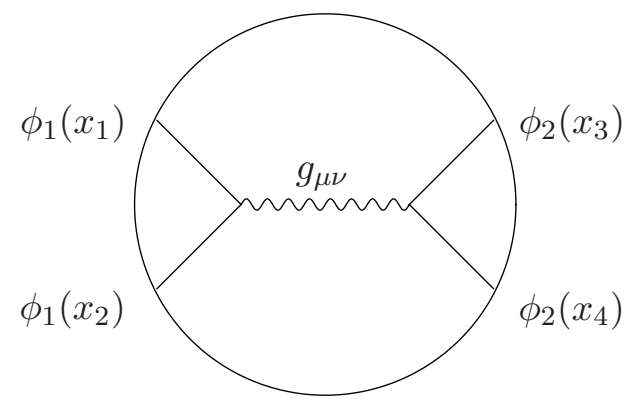

Figure 18. Graviton exchange Witten diagram.

From this we can compute the contribution of $T$ and all of its descendants in the double OPE $(12) \rightarrow(34)$ and we can write the result in terms of a "conformal partial wave." The final result is given in eqn (4.3) in [40]. Taking this result, expanding it in the (13) $\rightarrow(24)$ channel, and isolating the logarithmic singularity in that channel leads to

$$
\delta_{12}=-\frac{\Delta_{1} \Delta_{2}}{4 c}
$$

For e.g. $N=4 \mathrm{SYM}$ we have in the conventions of [40] $c=\frac{N^{2}-1}{4}$ so the anomalous dimension is

$$
\delta_{12} \approx-\frac{\Delta_{1} \Delta_{2}}{N^{2}}
$$

This has exactly the same dependence on $\Delta_{1}, \Delta_{2}$ and $N$ as one would expect from a bulk graviton exchange between two particles of mass $\ell \Delta_{1}$ and $\ell \Delta_{2}$ separated a distance $r \sim \ell$ i.e.

$$
G \frac{m_{1} m_{2}}{\ell^{2}}
$$

This strongly suggests that one should also be able to determine that to leading order in $1 / c$ the conformal dimension of $\boldsymbol{\Phi}$ behaves as

$$
\Delta(\Phi) \sim \Delta-a \frac{\Delta^{2}}{N^{2}}
$$

where $a$ is some number of order unity. The result (5.9) would support the bulk result that the neutron star becomes unstable at $\Delta \sim N^{2}$. It is however not straightforward to establish (5.9). One first needs to generalize the above computations to correlations functions involving $\Psi$ 's and their derivatives, and the latter are no longer conformal primaries. In addition, in these four-point functions not only $T$ but also bilinears in $\Psi$ and its derivatives 
will appear in the intermediate channel, so that additional conformal partial waves need to be included in the computation. These additional bilinear contributions to the double OPE are necessary in order to have a crossing-symmetric 4-point function at order $\frac{1}{c}$ : the conformal partial wave corresponding to $T_{\mu \nu}$ exchange is not crossing symmetric by itself. It has to be "dressed up" with contributions from the exchange of fermion bilinears in order to combine into a crossing-symmetric contribution which basically becomes the graviton exchange Witten diagram. Finally it would be important to clarify possible complications from the mixing of the composite operators with other operators of the same quantum numbers, once interactions are taken into account.

Apart from these complications it seems likely that the single graviton exchange in the bulk can be reproduced by the $T$ exchange in the double OPE. As we mentioned, the latter is fixed by conformal invariance and the Ward identities, so it can be reliably computed even at strong ' $t$ Hooft coupling if we know the conformal dimensions $\Delta_{1}, \Delta_{2}$ and the central charge $c$. Thus the gravitational force between two particles in the bulk can be understood on the boundary via the $T$ exchange, which is a universal property of CFTs. To compute the full backreaction in our scaling limit we need to sum over all pairs of particles, but also to include the effects of 3-body, 4-body etc. interactions in the bulk as we discussed in section 3.2. It is not clear to what extent these higher order forces can be explained on the boundary by some universal argument, as was the case for 2-body forces.

On the other hand, if we have established that the 2-particle force in the bulk is reproduced by $T$ exchange, then to compute the total backreaction from 2-particle forces we simply have to sum over all pairs (i.e. all pairs of single trace constituents of the multitrace operator $\mathbf{\Phi})$. This summation is an involved combinatoric problem but it has nothing to do with strong coupling effects in the gauge theory, so in principle it should be tractable. This part of the backreaction is the "Newtonian" limit of general relativity, in the sense that we ignore self-interactions of the gravitons. While this is not the full answer, we would like to emphasize that the existence of a maximum mass and the Chandrasekhar limit can also be estimated by treating gravity as Newtonian.

\subsection{Boundary description of the instability and the collapse}

The degenerate star represents a state in the conformal field theory with a large number of fermionic "glueballs", placed in a configuration with lowest possible energy. It is a high-density state at zero temperature. If we keep increasing the density by adding more glueballs, then at some point the state becomes unstable and presumably undergoes a phase transition towards a deconfined "quark-gluon plasma" thermal state, the dual of a black hole in the bulk.

It would be interesting to further explore the physics of this instability and the meaning of the tachyonic mode in the CFT. We expect that when the number of single trace operators multiplied together becomes very large the resultant operator mixes very strongly with other operators in the CFT and eventually with the operators dual to the black hole microstates, which have a very large entropy. Presumably the time scale set by the condensation of the tachyonic mode contains information about the thermalization time of the dual field theory. 


\section{$6 \quad$ Validity and embedding in string theory}

Our discussion so far has been general, without reference to a specific AdS/CFT setup. In this section we would like to discuss a couple of issues related to the validity of our approximations and the possibility of embedding our story in a precise holographic duality.

There are various possible sources of corrections to our analysis. We will address them in order of complexity as follows: first we will assume that the theory in the bulk consists only of a massive fermion and the graviton and check the validity of our approximations. Then we will include the effect of other massless fields, Kaluza-Klein modes on the sphere as well as stringy degrees of freedom.

\subsection{Toy model with a single fermionic field and the graviton}

A possible complication that we have not dealt with so far is that without a fermion conservation law, the number of fermions does not have to be constant in time as there may be processes under which fermions convert to gravitons and vice versa. Without a conservation law it is natural to expect that if we wait long enough then part of the fermions will be converted to gravitons and the final state will be a finite temperature thermal gas of gravitons and fermions in equilibrium.

Nevertheless we will now argue that the timescale for this thermalization can be made parametrically large, in such a way that the degenerate fermionic star is a good description of the system for quite a long timescale. This is based on the $\frac{1}{c}$ suppression of the interactions which are responsible for changing the number of fermions and gravitons. Let us now explain this point in more detail. We want to estimate the decay rate of the fermion gas to a mixed gas of fermions and gravitons. Notice that provided that the typical wavelength of the fermions is small (i.e. $\hat{\mu}(0)$ is large), which is true in our limit, this question can be studied locally by looking at a small box in AdS containing the fermionic gas and asking how quickly it converts to a mixture of fermions and gravitons. In other words it is a question about the validity of our equation of state, which can be analyzed even in flat space. So we consider a box of the gas at chemical potential $\mu$ and we try to estimate its "lifetime" $\tau$. The relevant scales in the problem are the fermion mass $m_{f}$, the chemical potential $\mu$ and the planck mass $m_{p}$ which controls the interactions. For simplicity we will only check the validity in the regime where $\mu \gg m_{f}$, in which the particles are extremely relativistic, and thus interactions are more energetic. If the approximation turns out to be reliable in this limit then we expect the same to be true for less energetic particles i.e. when $\mu \approx m_{f}$.

In this limit we have only two dimensionful parameters $\mu$ and $m_{p}$. We write the lifetime of the fluid as

$$
\tau=f\left(\frac{\mu}{m_{p}}\right) \frac{1}{\mu}
$$

for some function $f(x)$ of the dimensionless ratio involved in the problem. Our scaling limit corresponds to $\mu \sim c^{\frac{1}{d+1}}$ while in general $m_{p} \sim c^{\frac{1}{d-1}}$. In other words $x \rightarrow 0$. Let us assume that $f$ has a smooth limit in that regime and expand

$$
f(x)=x^{a}+\text { subleading }
$$


How can we determine the exponent $a$ ? This limit can also be understood as one where we keep $m_{p}$ fixed and send $\mu$ to zero. This is a limit of an infinitely dilute gas, where we can use Feynman diagrams to estimate the lifetime. As a typical example consider the leading Feynman diagram of two fermions going into two gravitons. From such diagrams it is easy to determine the dependence of the lifetime on Newton's constant and we find that to leading order $\tau$ goes like $\frac{1}{G}$, which implies $a=-d+1$, or the lifetime

$$
\tau \sim \frac{m_{p}^{d-1}}{\mu^{d}}+\text { subleading, } \quad \text { for } \quad \frac{\mu}{m_{p}} \rightarrow 0
$$

or

$$
\tau \ell \sim c^{\frac{1}{d+1}}+\text { subleading, } \quad \text { for } \quad c \rightarrow \infty
$$

Hence the star becomes long-lived (in AdS radius units) at large $c$. Similar scaling holds for other possible diagrams, ${ }^{30}$ if we assume that the correlators scale with $c$ in the way expected from a theory with a standard large $c$ expansion. ${ }^{31}$

\subsection{Including other modes}

The effects of these new fields can be roughly divided in two categories: first they may lead to a modification of the equation of state for the fermions (or even to a more drastic breakdown of the approximation of a fermionic fluid in the bulk) and second they may introduce new long range forces (for example electromagnetic ones if the fermions carry Rcharge). The effect of new long range forces is easier to deal with: one has to solve the TOV equations coupled to additional massless modes. For example we will discuss in more detail the inclusion of an electric field in the next section. As we will see the qualitative behavior of our system remains the same. In general the number of massless modes which can be sourced by the fermions will be small and we expect that generically the gravitational coupling will be the dominant one. ${ }^{32}$ So we do not expect that the inclusion of new long range forces will modify our results qualitatively.

On the other hand the corrections to the equation of state may be more drastic. To be concrete, we will first discuss whether these corrections are under control in the usual IIB $\mathrm{AdS}_{5} \times \mathrm{S}^{5}$ background dual to $\mathcal{N}=4 \mathrm{SYM}$. In this case we run into a difficulty. The mass of the fermion $\ell \Delta_{0}$ and the chemical potential $\mu$ scale like $N^{2 / 5} \ell$ in the limit we consider, which is parametrically larger than the typical mass of an excited string state $1 / \ell_{s} \sim\left(g_{s} N\right)^{1 / 4} \ell$ at weak string coupling. Therefore, we expect that massive string states will be produced by the interactions between the fermions. Notice that our scaling arguments of the previous section do not apply since we can also have diagrams of two fermions producing a single massive string state, for which the $\frac{1}{N}$ suppression is not sufficiently large. Moreover the

\footnotetext{
${ }^{30}$ Notice that in the regime where $\mu \gg m_{f}$ the particles are effectively massless, so from energy momentum conservation there are no diagrams with 2 incoming and 1 outgoing particles.

${ }^{31}$ This means that the connected correlator of $n$ "single trace" operators scales like $c^{\frac{2-n}{2}}$, in a normalization where the 2-point functions of single trace operators are of order one.

${ }^{32}$ One way to explain this expectation is that other long range forces will only couple to the "charges" of the basic fermion $\Psi$, while gravity couples to the mass of $\Psi$ as well as the kinetic energy of the fermions i.e. the "derivatives" acting on $\Psi$.
} 
Hagedorn growth in the density of string states (and thus in the density of possible final states) suggests that the longevity of the star cannot be guaranteed. Instead the star may quickly convert into a very complicated mess of stringy modes. ${ }^{33}$

An alternative AdS/CFT realization of our star would be in M-theory backgrounds, i.e in $\mathrm{AdS}_{4} \times \mathrm{S}^{7}$ or $\mathrm{AdS}_{7} \times \mathrm{S}^{4}$. The main advantage of these theories is that all new physics beyond supergravity is related to the single UV scale $m_{11}$, the eleven-dimensional Planck mass. Since we are working in a limit where all energy scales are parametrically smaller than $m_{11}$ we can safely ignore all M-theoretic objects beyond those of elevendimensional supergravity. To see this more precisely, let us from the start consider the full eleven-dimensional picture. In this case the massive fermion corresponds to a certain Kaluza-Klein mode of the supergraviton multiplet on the sphere. Now let us try some specific expressions. For simplicity we ignore factors of order 1 in the rest of this section. In the case of M-theory on $\mathrm{AdS}_{4} \times \mathrm{S}^{7}$ the AdS radius $\ell$ is related to the eleven-dimensional Planck mass $m_{11}$ by

$$
\ell=\frac{N^{1 / 6}}{m_{11}}
$$

where $N$ is the number of $M 2$ branes, or the units of 4 -form flux. In other words the central charge is $c=N^{3 / 2}$. It is not hard to see that in order to have nontrivial gravitational backreaction then we need an energy density (from the 11 dimensional point of view) which scales like

$$
\rho=N^{3 / 2} \frac{1}{\ell^{11}}
$$

So the $11 \mathrm{~d}$ chemical potential of our fluid is of the order

$$
\mu=N^{3 / 22} \frac{1}{\ell}
$$

Plugging into the formula for the lifetime for $d=10$ we find

$$
\tau \ell=\ell \frac{\left(m_{11}\right)^{9}}{\mu^{10}}+\ldots=N^{3 / 22}+\ldots
$$

so it increases with $N$ (though very slowly). This shows that if we have no new degrees of freedom at scales parametrically smaller than $m_{11}$ then in the large $N$ limit our fluid becomes stable.

Let us now explain why we do not expect any such degrees of freedom in M-theory on $\mathrm{AdS}_{4} \times \mathrm{S}^{7}$. Consider the sequence of compactifications of $\mathrm{M}$ theory on $\mathrm{AdS}_{4} \times \mathrm{S}^{7}$ labeled by the integer $N$. In the large $N$ limit the two scales that we are familiar with are the AdS scale $\ell$ and the $11 d$ planck mass $m_{11}$ which are related by (6.1). Let us now assume that there exists another mass scale, which lies somewhere between $1 / \ell$ and $m_{11}$ where new massive degrees of freedom appear, in analogy with the string scale in IIB. Let us call this scale $m_{\text {new }}$. We now consider how this scale behaves, relative to the AdS scale, in the large $N$ limit. In other words, what is the limit of the product $\ell m_{\text {new }}$ at large $N$ ? According to the AdS/CFT duality for this system we have

$$
\ell m_{n e w} \rightarrow 0
$$

\footnotetext{
${ }^{33}$ We would like to thank S. Minwalla for very helpful comments about the validity of our approximations.
} 
otherwise the statement that in the large $N$ limit we can approximate M-theory on $\mathrm{AdS}_{4} \times$ $\mathrm{S}^{7}$ by $11 \mathrm{~d}$ supergravity would not make sense.

So assuming this condition, we conclude that $m_{\text {new }}$ will necessarily have to be a new "UV scale" (i.e. a small length scale relative to the size of AdS). So we learn that in the large $N$ limit we have two UV scales $m_{\text {new }}$ and $m_{11}$. Notice that as we make $N$ larger and larger both of these scales move even more towards the UV, and become more and more tiny as length scales compared to the AdS scale. If in the large $N$ limit we had two such UV scales in M-theory on AdS it is natural to assume that the same would be true in flat space, since it is hard to imagine how the finite radius of AdS would introduce a new UV scale.

On the other hand we know that $11 \mathrm{~d}$ flat space M-theory has only one scale $m_{11}$, so $m_{\text {new }}$ has to be proportional to $m_{11}$ in the large $N$ limit and not parametrically separated from it. This is because there cannot be a free parameter to tune the ratio between the two as there are no moduli in 11d flat space M-theory. So essentially there is only one UV scale $m_{11}$ in the large $N$ limit. Then we can apply the arguments of the previous sections as before.

This argument suggests that our neutron star can probably be reliably embedded in M-theory on $\mathrm{AdS}_{4} \times \mathrm{S}^{7}$.

\section{Charged stars}

In this section we analyze the degenerate star in the case that the fermions are charged under a $\mathrm{U}(1)$ gauge field. One motivation to do so is the following: in most known examples of AdS/CFT dualities the light fields in the bulk correspond to chiral primaries on the boundary i.e. they have nontrivial R-charge. Hence the degenerate star will source the corresponding electromagnetic field, which in turn will induce an additional electromagnetic force on the fermions. We would like to understand to what extent the inclusion of these forces will modify our results. Another motivation is the recent analysis of bulk systems at finite charge density, usually in the presence of a black hole, in the context of "holographic superconductivity". 34

How can we determine the equilibrium configuration of a large number of charged fermions which are interacting gravitationally and electromagnetically? We would like to work directly in the hydrodynamic description and derive the analogue of the TOV equations for charged matter. First let us understand what is the equation of state that we have to use. Does the addition of charge modify the equation of state (2.22), (2.23), (2.24)? An equation of state is defined as the relation between various intensive quantities in the thermodynamic (infinite volume) limit. However when we have long-range forces sourced

\footnotetext{
${ }^{34}$ While this draft was being prepared the work [21] appeared, which has some overlap with the results of this section. That work focuses on bulk configurations of a charged fermionic fluid in the Poincare patch with planar sections (in contrast our stars are in global AdS and have spherical symmetry). Presumably the solutions of [21] can be recovered by taking the planar limit of the solutions constructed here, while scaling the fermion mass and charge appropriately at the same time. We would like to thank S. Hartnoll for discussions about their work.
} 
by the fluid it is impossible to take the infinite volume limit (the system suffers from the analogue of the Jeans instability).

To see what is the right way to include the effect of the charge let us remember how we incorporated the gravitational interactions of the fermions: we used the standard equation of state, but instead of simply solving the hydrodynamic equations $\nabla_{\mu} T^{\mu \nu}=0$ on a fixed background, we solved the Einstein equations using the fluid as a source, which led to the TOV equations. Similarly in the case of a charged fluid what we have to do is to solve the Maxwell-Einstein equations, where the fluid sources both the Einstein equations by its stress energy tensor and the Gauss law for the U(1) gauge field by its charge density.

\subsection{Charged TOV equations}

Here we derive the TOV equations for charged degenerate stars. We have the Einstein equations

$$
R_{\mu \nu}-\frac{1}{2} R g_{\mu \nu}+\Lambda g_{\mu \nu}=8 \pi G T_{\mu \nu}
$$

where the total energy-momentum tensor is now that of a perfect Fermi fluid plus that of the electromagnetic field

$$
T_{\mu \nu}=(\rho+p) u_{\mu} u_{\nu}+p g_{\mu \nu}+\frac{1}{4 \pi}\left(F_{\mu \rho} F_{\nu}^{\rho}-\frac{1}{4} g_{\mu \nu} F_{\rho \sigma} F^{\rho \sigma}\right)
$$

Gauss's law reads

$$
\partial_{\mu}\left(\sqrt{g} F^{\mu \nu}\right)=4 \pi \sqrt{g} J^{\nu}
$$

Turning on only the radial electric field $E(r)$, we have $F_{01}=-F_{10}=\sqrt{g_{t t} g_{r r}} E(r)$ as the only non-vanishing components of the electromagnetic field strength. Inserting this $T_{\mu \nu}$ into the Einstein equations merely has the effect of shifting the energy density and pressure by the following

$$
\rho \rightarrow \rho+\frac{E(r)^{2}}{8 \pi} \quad \text { and } \quad p \rightarrow p-\frac{E(r)^{2}}{8 \pi}
$$

This implies that the form of the first of the equations in (3.9) gets modified to

$$
\frac{d M}{d r}=V_{d-1} r^{d-1}\left(\rho+\frac{E^{2}}{8 \pi}\right)
$$

whereas the other two equations in (3.9) are only implicitly affected through $M(r)$, which enters the functions $A(r)$ and $B(r)$ in (3.8). Now, conservation of $T_{\mu \nu}$ yields

$$
\frac{d p}{d r}=-(p+\rho) \frac{A^{\prime}(r)}{A(r)}+\frac{E(r)}{4 \pi r}\left[(d-2) E(r)+r E^{\prime}(r)\right]
$$

The electric field enters the system of equations as a new variable, hence we need another equation in order to uniquely specify the solution. This comes in the form of Gauss' law, written as

$$
E^{\prime}(r)=-\frac{(d-2) E(r)}{r}+V_{d-1} \rho_{c h} B(r)
$$


where the charge density $\rho_{c h}(r)=q_{f} \mathbf{n}(r)$ for fermion charge $q_{f}$ and the number density $\mathbf{n}(r)$ can be obtained from the thermodynamic identity (2.23). All the primes denote derivatives with respect to $r$. Finally substituting eq. (7.5) into eq. (7.4) gives us the charged analog of the last equation in (3.9).

$$
\frac{d p}{d r}=-(p+\rho) \frac{A^{\prime}(r)}{A(r)}+\frac{V_{d-2}}{4 \pi} \rho_{c h} E(r) B(r)
$$

The boundary conditions remain the same as in section 3.4 for the uncharged case. Also eqs. (3.10) and (3.11) for the radius $R$ and total fermion number $N_{F}$ remain unchanged in form, except that the functions $M(r)$ and $B(r)$ therein are now replaced by their charged counterparts. We now proceed to solve this system of coupled differential equations numerically.

\subsection{Numerics of charged solutions}

Extending our results of the last section, we now look for numerical solutions of spherically symmetric and static, but charged TOV equations for degenerate stars in $\mathrm{AdS}_{5}$. As discussed in section 4.1 we are working with parameters scaled as $G_{5} / \ell^{3} \rightarrow 0$ and $M \ell \rightarrow \infty$. Therefore other physical quantities get the following rescaling

$$
\hat{E} \equiv\left(\frac{8 G_{5}}{\pi \ell^{3}}\right)^{1 / 2} E, \quad \hat{q}_{f} \equiv\left(\frac{8 G_{5}}{\pi \ell^{3}}\right)^{-3 / 10} q_{f}
$$

where the hatted quantities are kept fixed as $c \rightarrow \infty$. Notice that in this normalization the charge includes a factor of the $\mathrm{U}(1)$ coupling constant which goes like $G_{5}^{1 / 2}$. This means that in the normalization where the charge is integral it scales like $c^{1 / 5}$ which is consistent with the scaling $c^{1 / 5}$ of the fermion mass $m_{f}$ for the case where the fermions are (descendants of) chiral primaries.

\subsubsection{A charged degenerate star}

In this section we present solutions of typical degenerate stars comprised of charged fermions. In order to study the effects due to varying charge, we keep both the total fermion number $\hat{N}_{F}$ as well as the fermion mass $\hat{m}_{f}$ fixed. Then naturally, upon varying the charge of the star, we expect to see a variation in the total mass as well as the chemical potential profile. figure 19 shows the radial mass distribution for four stars with different charges. The mass profiles approach the total mass as $r / \ell \rightarrow \infty$. Notice that turning on fermion charge gradually increases the mass even though the total number of fermions is held fixed. This is what we expect from eq. (7.3), where the square of the electric field enters the mass integral. What happens here is that electrostatic repulsion acts against gravitational attraction and that reduces the binding energy compared to the uncharged case.

In figure 20 we plot radial profiles of the local Fermi momentum in the bulk for the same set of charges as in figure 19. The radius of the star can be read-off from the $x$ intercept, where the pressure, energy and number densities all vanish. Compared to the uncharged star (denoted by the blue curve), we see that increasing fermion charge has the 


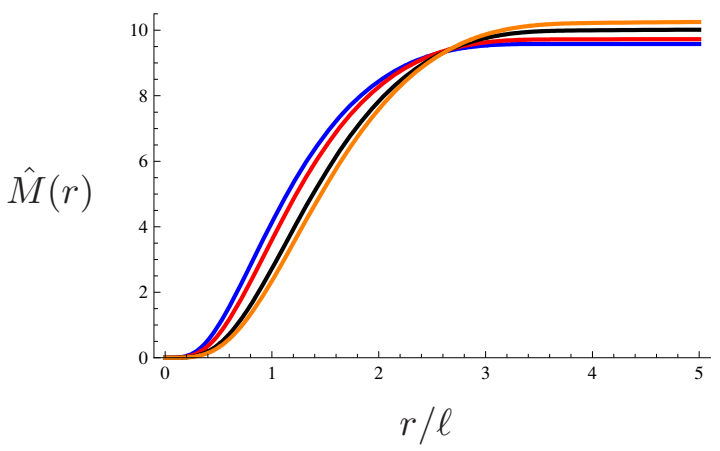

Figure 19. Radial mass distributions of charged degenerate stars. Blue profile denotes the uncharged case; red, black, orange denote profiles with succesively increasing charges.

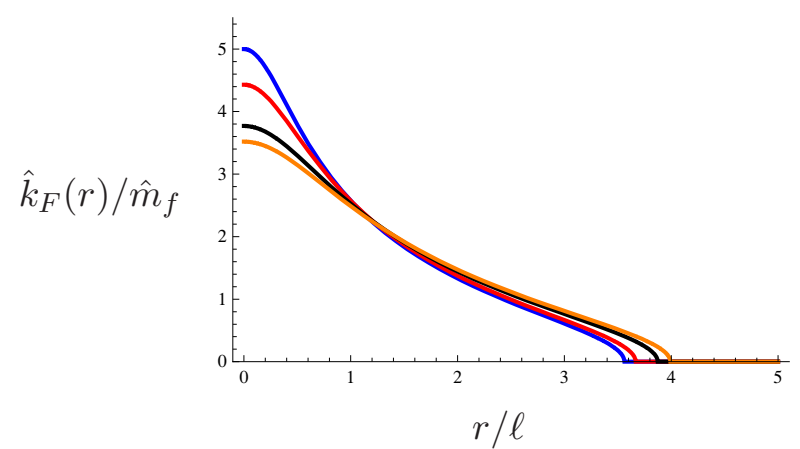

Figure 20. Radial functions of Fermi momentum in ascending order of charge, with uncharged case shown in blue.

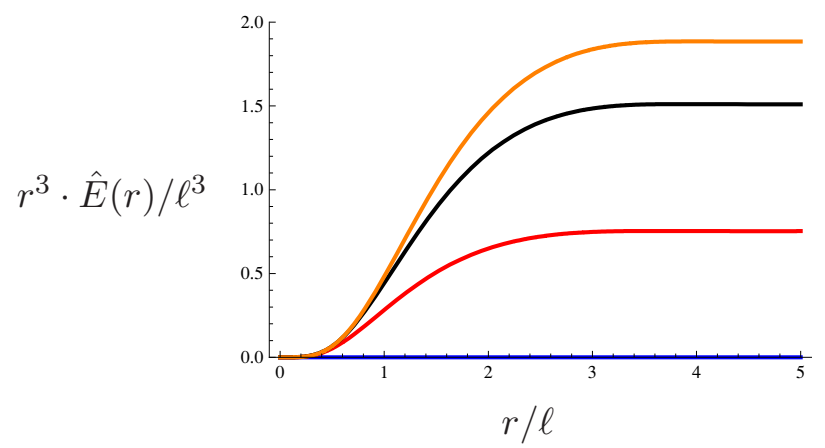

Figure 21. Electric fields inside and outside the stellar mass, scaled by $r^{3}$. Flat line (blue) denotes uncharged case, orange curve represents maximally charged case.

effect of increasing the overall size of the star. Also the fermionic momentum at the core of charged stars is less than that of the uncharged one. Both these effects can be understood as the electrostatic repulsion acting against the gravitational attraction. Remarkably, as we see from these plots, this cancellation effect is only small, given that we have determined solutions for a wide range of fermionic charges.

Figure 21 gives the solution of the electric field of the same stars, scaled by $r^{3}$. The blue profile is zero everywhere, as it should. For the other profiles, we note that they exactly flatten outside the radius of the respective stars. For a spherically symmetric metric in five dimensions, this means that the electric field outside the star behaves like one resulting 


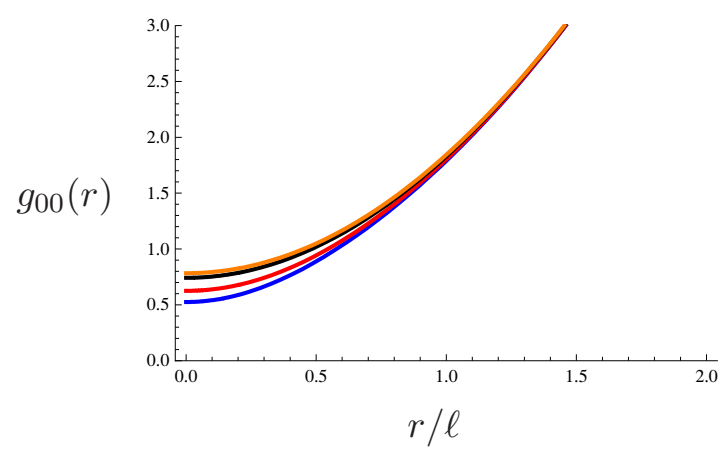

Figure 22. Gravitational potential of the charged degenerate stars.

from a point charge that falls off as $1 / r^{3}$. Finally in figure 22 we see the $g_{00}$ component of the metric. As expected, the minima of the charged solutions lie slightly above that of the uncharged.

What we notice from the results presented in this section is that deviations from the uncharged star upon tuning on fermion charge are relatively only a small fraction and comparable to the difference between the self-gravitating and unbackreacted star in the previous section.

\subsubsection{Family of charged TOV solutions and critical mass}

We now study the characteristics of a family of charged solutions. This will help us identify stable solutions for static, spherically symmetric charged degenerate stars. Furthermore this analysis also reveals some interesting features of the solution into the unstable phase (after the critical point). The results are presented in figure 23. Here we have four profiles in order of increasing fermion charges; violet being the uncharged case and green being the maximum charge. The fermion mass $\hat{m}_{f}$ has been held fixed throughout. Each point on these profiles represents a star with a given central chemical potential $\hat{\mu}(0)$. Knowing this, one can easily compute the energy density at the core of a star $\hat{\rho}(0)$, which is plotted on the $x$-axis with a logarithmic scaling.

On the vertical axis of figure 23 we have the mass. Just as in figure 7 in section 4.1.2 once again we clearly observe the existence of a critical mass, seen at the overall maximum of each profile. This is the Chandrasekhar or Oppenheimer-Volkoff limit for charged stars. After this limit, the solutions are unstable against radial perturbations for the same reasons as discussed in section 4.2. Compared to the uncharged case, we see that the critical mass rapidly increases upon turning on a charge. The figure also shows that the critical chemical potential $\hat{\mu}_{c}$ gradually increases and so does the amplitude of oscillations in the unstable region like those discussed in section 4.4.2. In conclusion, we observe that many qualitative features regarding the onset of gravitational collapse discussed in section 4.2 remain valid also for charged degenerate stars.

These observations also suggest that the holographically dual CFT picture for charged stars is a relatively straightforward extension of the uncharged case. In addition to the $T_{\mu \nu}$ exchange between single-trace constituents we also have to include the exchange of the conserved current $J_{\mu}$ dual to the $\mathrm{U}(1)$ gauge field in the bulk. 


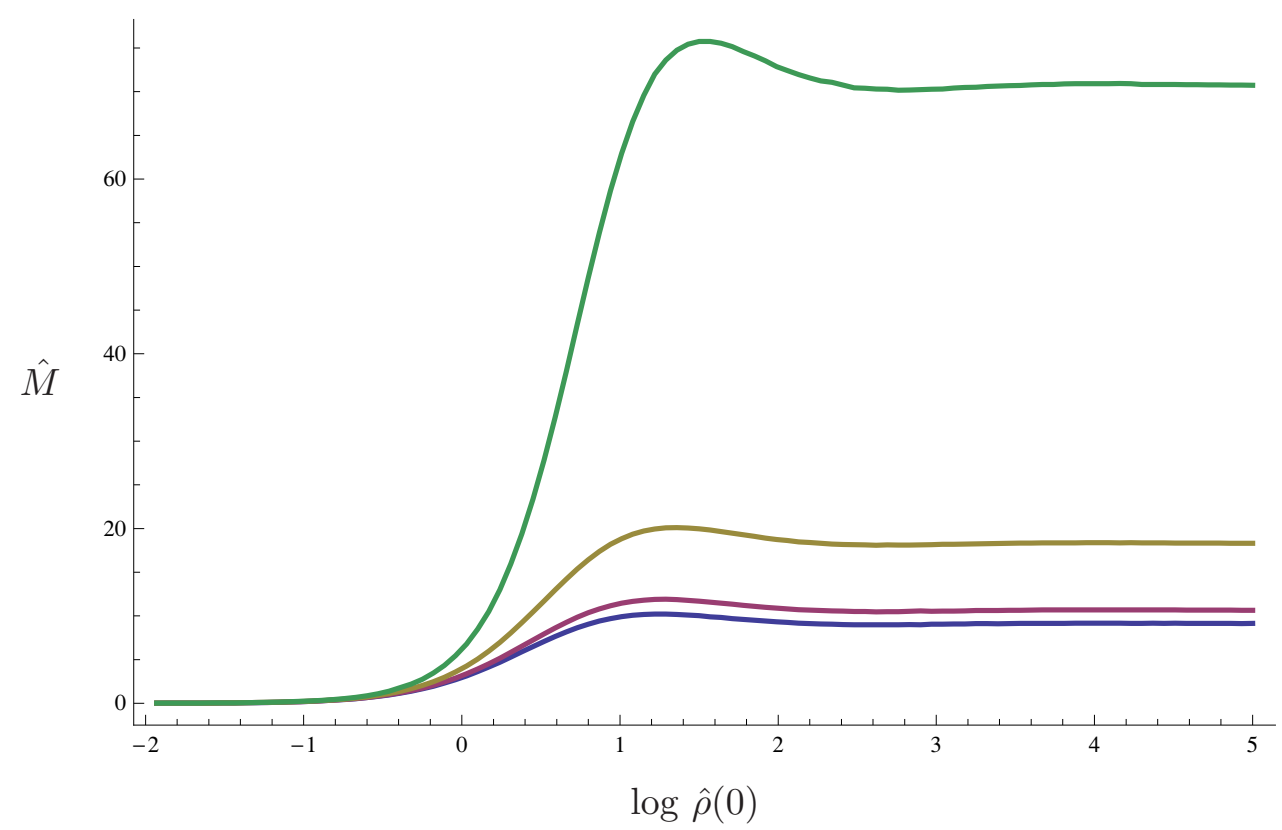

Figure 23. Total mass vs central energy density (logarithmically scaled) for a family of stars with increasing values of fermion charge, from uncharged case (violet) to maximally charged (green).

Finally let us also note that the charge of the star cannot be increased indefinitely, keeping other parameters fixed. We have checked numerically, that in that event the solution itself breaks down. This can be understood in the following way. From the discussion in section 7.2.1 above we have seen that the electrostatic force of the star acts against its self-gravity. Once this repulsion becomes large enough to overtake self-gravity, it inhibits the formation of a star. On the other hand, there might be a double scaling limit which would allow us to construct arbitrarily large charged stars and to take the "Poincare limit" of global AdS, which would most likely connect to the solutions considered in [21]. It would be interesting to explore this further.

\subsubsection{Attractor fixed points}

Another feature of the limiting solutions deep into the unstable regime is the existence of attractor fixed points in the solution space of TOV equations is shown in figure 24, where the mass has been plotted versus the radius of the star for a family of stars each with a specific central chemical potential $\hat{\mu}(0)$. If we interpret this in a dynamical systems language, the radial direction plays the role of the time parameter. In the scaling limit $\hat{\mu}(0) \rightarrow \infty$, the solutions remarkably spiral towards the attractor fixed points of the TOV equations. In figure 24, the different profiles denote solutions with different fermion charges, with the violet curve being the uncharged case and the green profile being maximally charged. These spirals are precisely the mass (and similarly radius) oscillations that we discuss in section 4.4.2 below. Here we see note that these spiraling attractors/mass oscillations are a generic feature of the TOV system of equations, both with and without U(1) charges. Figure 25 shows a blow-up near one of the fixed points. In these plots the fermion mass $\hat{m}_{f}$ was held fixed. Upon reversing the set-up to vary $\hat{m}_{f}$ and keep the fermion charge 


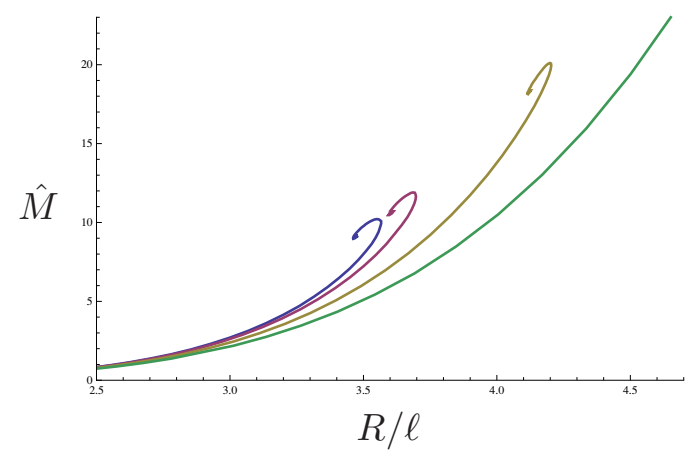

Figure 24. Mass vs radius of a family of stars for increasing fermion charges from blue curve (uncharged) to green (maximum charge).

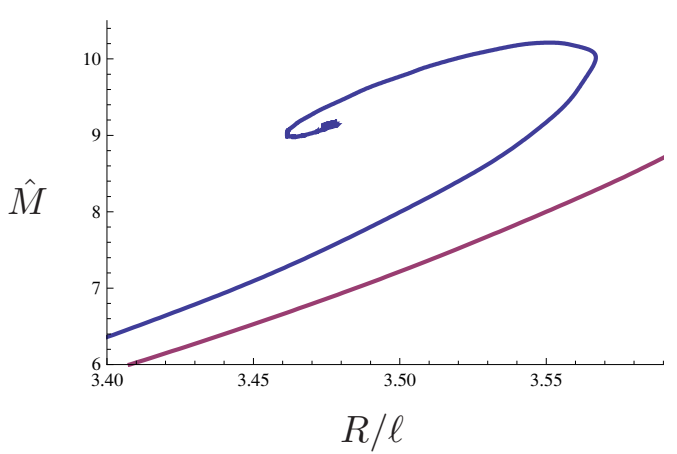

Figure 25. Zooming-in the mass vs stellar radius profile near the fixed point. The blue curve denotes a family of uncharged stars.

fixed, we have also observed similar spiraling attractors. In this sense, the fermion mass and charge fully specify the parameter space of all fixed points.

\section{Thermal graviton star}

In this section we will study the thermal version of a star in an $\operatorname{AdS}_{d+1} \times \mathrm{S}^{k}$ background. We will consider a thermal gas of all ${ }^{35}$ supergravity modes at sufficiently high temperature so that they backreact to the geometry. Our solution will be the analogue of the radiation star [32], including certain modifications due to the internal sphere $\mathrm{S}^{k}$.

The main motivation for considering the thermal star is the following: if we start with a generic, sufficiently complicated, initial state in AdS we expect that at late times ${ }^{36}$ the system will be approximately described by a thermal density matrix of an ensemble with the same values of conserved charges (such as energy or R-charge) as the initial state. This final thermal state may be a black hole in AdS, or a thermal gas of all supergravity fields depending on the initial state. We want to study the thermal gas endpoint in the case where the temperature is high enough for the gas to backreact.

\footnotetext{
${ }^{35}$ In this section when we refer to a "graviton star" we refer to a system where all massless supergravity fields have been turned on thermally.

${ }^{36}$ Depending on the choice of initial state the thermalization may be slow in units of the AdS scale, in the large $N$ limit, as we argued in the previous section. However if we wait long enough the system will eventually thermalize.
} 
Moreover, the thermal star may describe a "superheated" phase of certain gauge theories as we now explain. Large $N$ gauge theories with classical gravity duals undergo deconfinement phase transitions [41], which are the analogue of the Hawking-Page phase transition between a gas of gravitons at low temperature and a black hole at high temperature. This phase transition takes place at a temperature $T_{H P}$ which is of order one (i.e does not scale with $N$ ). The low temperature phase has energy of order $N^{0}$ while the high temperature has energy of order $N^{2}$. The jump of energy between the two phases indicates that the phase transition is of first order. What would happen if we considered the same system in the microcanonical ensemble? Notice that the thermal gas phase, while thermodynamically subdominant, is locally stable even at $T>T_{H P}$. This means that if we work in the microcanonical ensemble we can push the thermal graviton gas into a superheated phase by pumping in energy so that the effective temperature $T$ of the gas will go above the Hawking-Page temperature $T_{H P} \cdot{ }^{37}$

Let us consider what happens to this superheated phase as we increase the temperature $T$. From general intuition we expect that the temperature cannot be increased indefinitely since the graviton gas will undergo gravitational collapse, if sufficiently heavy. The energy of a thermal gas in $\mathrm{AdS}_{d+1}$ grows like $T^{d+1}$ so it will backreact to the geometry when $G T^{d+1} \ell^{2} \sim 1$ where $G$ is Newton's constant. In terms of the central charge we find that gravitational backreaction of the thermal gas will start to take place when $T \sim c^{1 /(d+1)} \ell^{-1}$ in the large $c$ limit. ${ }^{38}$ So in theories where there is no other scale up to the Planck mass (for example in theories without a "string scale" ${ }^{39}$ ) we expect that the superheated thermal gas phase will indeed persist up to temperatures of the order where gravitational backreaction will become important. We want to compute the effect of the backreaction and understand thermodynamic properties of the superheated phase, for example its equation of state $E(T)$.

To compute the backreaction of a thermal gas we have to solve the Einstein equations coupled to the stress tensor of a fluid at temperature $T$. For massless fields in the bulk the relevant equation of state is that of radiation. If the massless fields have spin we simply have to multiply the energy and pressure by the appropriate number of polarizations. This means that a thermal graviton star in $\mathrm{AdS}_{d+1}$ is the same as the radiation star of [32], apart from a difference in a numerical coefficient in the equation of state, relating the energy density to the temperature, due to the number of graviton polarizations. Then we do indeed see that there is a maximum temperature beyond which the superheated phase ceases to exist.

In this section we would like to repeat the computation of [32] in backgrounds of the form $\mathrm{AdS}_{d+1} \times \mathrm{S}^{k}$ i.e with an internal sphere, since they are more relevant for AdS/CFT. For this purpose we start directly with the $d+k+1$ dimensional supergravity equations, sourced by a $d+k+1$ dimensional radiation fluid. After describing the structure of the

\footnotetext{
${ }^{37}$ Of course this would not make sense in the canonical ensemble.

${ }^{38}$ Notice that this is parametrically smaller than the Planck scale in the bulk which grows like $m_{P} \sim$ $c^{\frac{1}{d-1}} \ell^{-1}$.

${ }^{39}$ As we discussed in the previous section, this phase is not relevant for the $\mathcal{N}=4 \mathrm{SYM}$, since the required temperature is much hotter than the Hagedorn temperature but it might be realizable in M-theory backgrounds.
} 
resulting equations we give a concrete example by solving these equations numerically for the specific case of M-theory on $\mathrm{AdS}_{4} \times \mathrm{S}^{7}$.

Another motivation to consider a backreacted thermal gas on $\mathrm{AdS}_{d+1} \times \mathrm{S}^{k}$ background, thus generalizing the work of [32], is in order to clarify the effect of the KK modes on the $\mathrm{S}^{k}$ sphere. In the previous sections of the paper we were working in the $d+1$ dimensional reduced theory on $\mathrm{AdS}_{d+1}$, so a natural question was how much do the other KK modes on the sphere modify our results. To answer this question qualitatively it is perhaps easier to go directly to the full $d+k+1$-dimensional theory where there is no issue about the KK reduction. As we will see the qualitative features of the solutions are similar to the ones we have been discussing.

\subsection{The equations of motion}

We start by considering a general $\mathrm{AdS}_{d+1} \times \mathrm{S}^{k}$ compactification. We take the action in $d+k+1$ dimensions to be

$$
S=\frac{1}{16 \pi \widetilde{G}} \int d x \sqrt{\widetilde{g}}\left(\widetilde{R}-\frac{1}{2} F^{2}\right)
$$

where for the $(d+1)$-form we have defined $F^{2}=\frac{1}{(d+1) !} F_{\mu_{1} \ldots \mu_{d+1}} F^{\mu_{1} \ldots \mu_{d+1}}$. The possible presence of Chern-Simons terms will not be relevant for our static spherically symmetric solutions. The equations of motion are

$$
\widetilde{R}_{\mu \nu}-\frac{1}{2} \widetilde{g}_{\mu \nu} \widetilde{R}=\frac{1}{2 d !} F_{\mu \mu_{2} \ldots \mu_{d+1}} F_{\nu}^{\mu_{2} \ldots \mu_{d+1}}-\frac{1}{4} F^{2} \widetilde{g}_{\mu \nu}
$$

To fix the asymptotic form of the metric we first look for an $\mathrm{AdS}_{d+1} \times \mathrm{S}^{k}$ solution, without the radiation fluid, of the form

$$
d s^{2}=\ell^{2} d s_{\mathrm{AdS}_{d+1}}^{2}+R^{2} d \Omega_{k}^{2}
$$

where $d s_{\mathrm{AdS}_{d+1}}^{2}$ and $d \Omega_{k}^{2}$ are the metrics of the unit radius $\mathrm{AdS}_{d+1}$ and $\mathrm{S}^{k}$ respectively. We take the form $F$ to be proportional to the volume form of $\mathrm{AdS}_{d+1}$. We introduce the effective $d+1$ dimensional cosmological constant $\Lambda$ as

$$
\frac{F^{2}}{4}=\frac{(d+k-1)}{(d-1)(k-1)} \Lambda
$$

and then from the equations of motion we find that

$$
\ell^{2}=-\frac{d(d-1)}{2 \Lambda} \quad, \quad R^{2}=-\frac{(d-1)(k-1)^{2}}{2 d \Lambda}
$$

In the presence of the radiation fluid the $d+k+1$ dimensional equations of motion (8.1) are modified as

$$
\widetilde{R}_{\mu \nu}-\frac{1}{2} \widetilde{g}_{\mu \nu} \widetilde{R}=\frac{1}{2 d !} F_{\mu \mu_{2} \ldots \mu_{d+1}} F_{\nu}^{\mu_{2} \ldots \mu_{d+1}}-\frac{1}{4} F^{2} \widetilde{g}_{\mu \nu}+8 \pi \widetilde{G} T_{\mu \nu}^{\text {fluid }}
$$

where the stress energy tensor of the fluid in $d+k+1$ dimensions is

$$
T_{\mu \nu}^{\text {fluid }}=(\widetilde{\rho}+\widetilde{p}) \widetilde{u}_{\mu} \widetilde{u}_{\nu}+\widetilde{p} \widetilde{g}_{\mu \nu}
$$


with the equation of state for radiation

$$
\widetilde{p}=\frac{1}{d+k} \widetilde{\rho}
$$

The energy density is related to the temperature in $d+k+1$ dimensions by $\rho=\sigma T^{d+k+1}$ where the constant $\sigma$ is proportional to total number of bosonic degrees of freedom of the theory. The velocity satisfies $\widetilde{u}_{\mu} \widetilde{u}^{\mu}=-1$ with respect to the metric $\widetilde{g}_{\mu \nu}$.

We now focus on static configurations which also respect the $\mathrm{SO}(k+1)$ symmetry of the $\mathrm{S}^{k}$. The size of $\mathrm{S}^{k}$ will not be constant in the radial direction, therefore we consider the following ansatz for the metric in $d+k+1$ dimensions

$$
d s^{2}=e^{-\frac{2 k}{d-1} \phi\left(x^{\mu}\right)} g_{\mu \nu}\left(x^{\mu}\right)+R^{2} e^{2 \phi\left(x^{\mu}\right)} d \Omega_{k}^{2}
$$

where $g_{\mu \nu}$ is the $d+1$ dimensional metric and the form $F$ is still proportional to the volume form of the $d+1$ dimensional space. To proceed we will write the equations in terms of the $d+1$ dimensional metric $g$ and the scalar $\phi$. The $d+1$ dimensional Newton's constant $G$ is defined by the relation

$$
\frac{1}{16 \pi G}=\frac{R^{k} V_{k}}{16 \pi \widetilde{G}}
$$

where $V_{k}$ is the volume of the unit $\mathrm{S}^{k}$. The velocity $\widetilde{u}_{\mu}$ is normalized to square to -1 with respect to the metric $\widetilde{g}_{\mu \nu}$, so we introduce

$$
u_{\mu}=e^{\frac{k}{d-1} \phi} \widetilde{u}_{\mu}
$$

which satisfies $g^{\mu \nu} u_{\mu} u_{\nu}=-1$. We also define effective $d+1$ dimensional pressure and density

$$
p=\left(R^{k} V_{k}\right)^{-1} e^{-\frac{2 k}{d-1} \phi} \widetilde{p}, \quad \rho=\left(R^{k} V_{k}\right)^{-1} e^{-\frac{2 k}{d-1} \phi} \widetilde{\rho}
$$

After some algebra we find that (8.1) can be written as $d+1$ dimensional equations in the form

$$
\begin{aligned}
R_{\mu \nu}-\frac{1}{2} g_{\mu \nu} R+\Lambda g_{\mu \nu}= & \frac{k(d+k-1)}{(d-1)}\left(\partial_{\mu} \phi \partial_{\nu} \phi-\frac{1}{2} g_{\mu \nu}(\partial \phi)^{2}-V(\phi) g_{\mu \nu}\right) \\
& +8 \pi G\left[(\rho+p) u_{\mu} u_{\nu}+p g_{\mu \nu}\right] \\
\square \phi= & V^{\prime}(\phi)-8 \pi G p
\end{aligned}
$$

with

$$
V(\phi)=-\Lambda \frac{d}{k-1}\left(\frac{e^{-2 \frac{k d}{d-1} \phi}}{k d}-\frac{e^{-2 \frac{k+d-1}{d-1} \phi}}{d+k-1}\right)-\Lambda \frac{d-1}{k(d+k-1)}
$$

and

$$
p=\frac{1}{d+k} \rho
$$




\subsection{The graviton star solution}

Now we consider a spherically symmetric ansatz for the $d+1$ dimensional metric

$$
d s^{2}=-e^{2 \chi(r)-2 \beta(r)} d t^{2}+e^{2 \beta(r)} d r^{2}+r^{2} d \Omega_{d-1}^{2}
$$

with $u_{t}=e^{\chi(r)-\beta(r)}$ and $\phi(r), p(r), \rho(r)$ functions of $r$ only. The scalar equation becomes

$$
\partial_{r}^{2} \phi+\left(\chi^{\prime}-2 \beta^{\prime}+\frac{d-1}{r}\right) \partial_{r} \phi=e^{2 \beta} V^{\prime}(\phi)-e^{2 \beta} 8 \pi G p
$$

The $t t$ equation gives

$$
\frac{d-1}{2 r^{2}}\left((d-2)\left(e^{2 \beta}-1\right)+2 r \beta^{\prime}\right) e^{-2 \beta}+\frac{d(d-1)}{2 \ell^{2}}=\frac{k(d+k-1)}{(d-1)}\left(\frac{1}{2}\left(\partial_{r} \phi\right)^{2} e^{-2 \beta}+V(\phi)\right)+8 \pi G \rho
$$

Defining

$$
e^{-2 \beta}=1+\frac{r^{2}}{\ell^{2}}-C_{d+1} \frac{M(r)}{r^{d-2}}
$$

with $C_{d+1}=\frac{16 \pi G}{(d-1) V_{d-1}}$ we find

$$
M^{\prime}=V_{d-1} r^{d-1}\left[\rho+\frac{1}{8 \pi G} \frac{k(k+d-1)}{(d-1)}\left(\frac{1}{2}\left(\partial_{r} \phi\right)^{2} e^{-2 \beta}+V(\phi)\right)\right]
$$

Adding the $t t$ and $r r$ equations we find

$$
\chi^{\prime}=\frac{r}{d-1}\left(\frac{k(d+k-1)}{(d-1)} \frac{1}{2}\left(\partial_{r} \phi\right)^{2}+8 \pi G(\rho+p) e^{2 \beta}\right)
$$

Finally from the conservation equation of the total $T_{\mu \nu}$ one can show that the effective $d+1$ dimensional density and pressure are

$$
\rho(r)=\frac{\widetilde{\rho_{0}}}{R^{k} V_{k}} e^{-\frac{k(d+k+1)}{d-1} \phi_{0}+(d+k+1) \chi_{0}} e^{\frac{k(d+k-1)}{d-1} \phi(r)-(d+k+1)(\chi(r)-\beta(r))}, \quad p(r)=\frac{1}{d+k} \rho(r)
$$

where $\widetilde{\rho}_{0}$ is the $d+k+1$ dimensional density at the center.

These equations can be used to find solutions numerically. We have to specify the $d+k+1$ dimensional density $\widetilde{\rho}_{0}$ at the center and the value $\phi_{0}$ of the scalar field and then integrate outwards. The value $\chi_{0} \equiv \chi(0)$ at the center can be determined as follows: we notice that the differential equations are invariant under a constant shift of $\chi$ which corresponds to an overall rescaling of the time coordinate. By demanding that at infinity the metric is written in the standard parametrization of AdS space we have to impose $\chi(\infty)=0$. Using the invariance under constant shifts of $\chi$ this can always be achieved by an appropriate choice of $\chi_{0}$. So $\chi_{0}$ is not really an independent parameter that we can tune. At this stage it seems that the independent initial data are given by the two numbers $\left(\widetilde{\rho}_{0}, \phi_{0}\right)$. However we also have another boundary condition that we have to satisfy. At large $r$ the scalar field has to go to the minimum of the potential $\phi=0$, otherwise we would not have an asymptotically AdS space. This shows that the pairs of initial data $\left(\widetilde{\rho}_{0}, \phi_{0}\right)$ are actually not independent. For every $\widetilde{\rho}_{0}$ there is a value $\phi_{0}$ such that after 


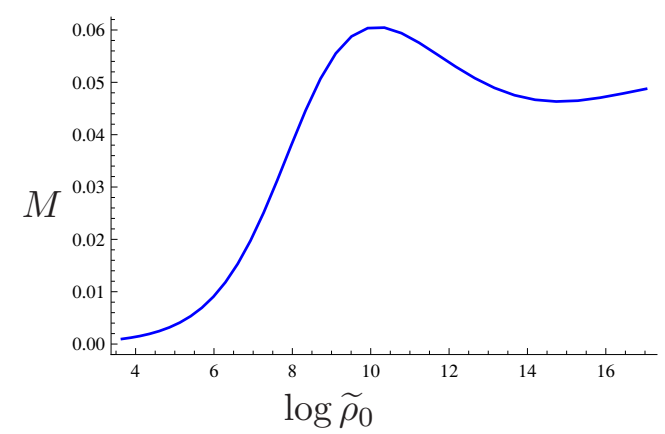

Figure 26. Mass of a thermal star in $\mathrm{AdS}_{4} \times \mathrm{S}^{7}$ as a function of the 11-dimensional energy density $\widetilde{\rho}_{0}$ at the center.

evolving these initial data we get an asymptotically AdS space. Solving this "shooting problem" numerically we can determine a one-parameter family of graviton-star solutions parametrized by the density at the center $\widetilde{\rho}_{0}$.

In figure 26 we show the mass of the star as a function of $\widetilde{\rho}_{0}$. We notice that the qualitative features are similar to those of a radiation star [32] in $\mathrm{AdS}_{4}$. There is a critical central density $\widetilde{\rho}_{c}$ beyond which the mass of the star starts to decrease signaling a Chandrasekhar-type instability. Solutions with $\widetilde{\rho}_{0}>\widetilde{\rho}_{c}$ are unstable under radial perturbations and presumably collapse. The natural endpoint of this collapse is an eleven dimensional Schwarzschild black hole in thermal equilibrium with its Hawking radiation. ${ }^{40}$ It would be interesting to study these thermal stars in more detail and their possible relevance for finite temperature gauge theories.

\section{Discussions}

In this paper we studied the holographic interpretation of degenerate fermionic stars in anti de Sitter space. Clearly our work leaves many important questions unanswered and is only a first step in a potentially interesting direction. Let us now mention some possible further directions.

It may be interesting to study the dynamical process of collapse towards a black hole by computing numerically the time-dependent solution in the case that the mass of the star exceeds the Chandrasekhar bound. In principle this bulk computation is straightforward, though perhaps tedious, and it might be useful for answering certain questions about the dual deconfinement transition in the boundary CFT. Along these lines it might also be interesting to explore possible connections with critical scaling during the collapse. The Choptuik scaling has been discussed in the context of AdS/CFT in recent works [42-45]. The analogue of Choptuik scaling has been observed in the (driven) collapse of neutron stars $[46,47],{ }^{41}$ so it would presumably be relevant for the collapse of our star and might have an interesting interpretation in the dual gauge theory.

\footnotetext{
${ }^{40}$ We are always working in the microcanonical ensemble so the total energy is conserved.

${ }^{41}$ We would like to thank L. Alvarez-Gaume for bringing this to our attention and for useful comments.
} 
Another interesting direction would be the computation of boundary correlation functions in the presence of the star. It would be useful to understand how the bulk Fermi surface is visible in terms of the boundary correlators and compare with works [17-20] where the fermionic correlators are evaluated in the background of a black hole (see also [48] for related issues). Let us notice that our star (at least the one without charge) can only exist in global AdS coordinates and not in the Poincare patch. In terms of the boundary theory the star would describe a low-temperature sector of the theory, at temperatures below the Hawking-Page transition and the black hole formation. So in a sense it would be the analogue of the low-temperature phase of the systems studied in the aforementioned works, at low temperature/chemical potential.

It would be useful to further clarify issues related to the validity of our approximations. While we tried to address some of these issues, a more carefully analysis would be desirable. In particular it it would be interesting to settle conclusively whether a degenerate fermionic star can be reliably realized in a known AdS/CFT duality.

Finally, it is clear that the boundary CFT treatment in our paper was on a rather basic level. We hope that more can be done in the direction of analyzing the interacting gas of fermions directly on the CFT side, perhaps under suitable simplifying assumptions about the spectrum of the theory and the strength of interactions, extending the basic estimates that we sketched in this paper. Furthermore it would be interesting to understand how to translate the dynamical evolution of the collapsing star (after the relevant solution has been calculated in the bulk) into useful information about the thermalization and deconfinement of the gas of glueballs in the gauge theory side. We leave these questions for future work.

\section{Acknowledgments}

We would like to thank O. Aharony, L. Alvarez-Gaume, I. Bena, S. Bhattacharyya, E. Floratos, S. Hartnoll, S. Hellerman, G. Horowitz, V. Hubeny, N. Iizuka, E. Kiritsis, J. Maldacena, P. McFadden, S. Minwalla, B. Nienhuis, H. Ooguri, M. Rangamani, L. Rastelli, K. Schalm, K. Schoutens, M. Shigemori, A. Strominger, M. Taylor, S. Trivedi, and H. Verlinde for discussions. This work was partly supported by the Foundation of Fundamental Research on Matter (FOM). K.P. would like to thank the organizers of the Kolymbari meeting, the university of Crete, the theory group at CERN and the Saclay string theory group for hospitality during various stages of this work.

Open Access. This article is distributed under the terms of the Creative Commons Attribution Noncommercial License which permits any noncommercial use, distribution, and reproduction in any medium, provided the original author(s) and source are credited.

\section{References}

[1] J.M. Maldacena, The large- $N$ limit of superconformal field theories and supergravity, Adv. Theor. Math. Phys. 2 (1998) 231 [Int. J. Theor. Phys. 38 (1999) 1113] [hep-th/9711200] [SPIRES]. 
[2] J.R. Oppenheimer and G.M. Volkoff, On massive neutron cores, Phys. Rev. 55 (1939) 374 [SPIRES].

[3] S.F. Ross and R.B. Mann, Gravitationally collapsing dust in $(2+1)$-dimensions, Phys. Rev. D 47 (1993) 3319 [hep-th/9208036] [SPIRES].

[4] Y. Peleg and A.R. Steif, Phase transition for gravitationally collapsing dust shells in (2+1)-dimensions, Phys. Rev. D 51 (1995) 3992 [gr-qc/9412023] [SPIRES].

[5] U.H. Danielsson, E. Keski-Vakkuri and M. Kruczenski, Vacua, propagators and holographic probes in AdS/CFT, JHEP 01 (1999) 002 [hep-th/9812007] [SPIRES].

[6] U.H. Danielsson, E. Keski-Vakkuri and M. Kruczenski, Spherically collapsing matter in AdS, holography and shellons, Nucl. Phys. B 563 (1999) 279 [hep-th/9905227] [SPIRES].

[7] S.B. Giddings and S.F. Ross, D3-brane shells to black branes on the Coulomb branch, Phys. Rev. D 61 (2000) 024036 [hep-th/9907204] [SPIRES].

[8] U.H. Danielsson, E. Keski-Vakkuri and M. Kruczenski, Black hole formation in AdS and thermalization on the boundary, JHEP 02 (2000) 039 [hep-th/9912209] [SPIRES].

[9] V. Balasubramanian, J. de Boer, E. Keski-Vakkuri and S.F. Ross, Supersymmetric conical defects: towards a string theoretic description of black hole formation, Phys. Rev. D 64 (2001) 064011 [hep-th/0011217] [SPIRES].

[10] S.B. Giddings and A. Nudelman, Gravitational collapse and its boundary description in AdS, JHEP 02 (2002) 003 [hep-th/0112099] [SPIRES].

[11] G.L. Alberghi and R. Casadio, On the gravitational collapse in Anti-de Sitter space-time, Phys. Lett. B 571 (2003) 245 [gr-qc/0306002] [SPIRES].

[12] V.E. Hubeny, H. Liu and M. Rangamani, Bulk-cone singularities \&3 signatures of horizon formation in AdS/CFT, JHEP 01 (2007) 009 [hep-th/0610041] [SPIRES].

[13] S. Lin and E. Shuryak, Toward the AdS/CFT gravity dual for high energy collisions. 3. Gravitationally collapsing shell and quasiequilibrium, Phys. Rev. D 78 (2008) 125018 [arXiv: 0808.0910] [SPIRES].

[14] P.M. Chesler and L.G. Yaffe, Horizon formation and far-from-equilibrium isotropization in supersymmetric Yang-Mills plasma, Phys. Rev. Lett. 102 (2009) 211601 [arXiv:0812.2053] [SPIRES].

[15] S. Bhattacharyya and S. Minwalla, Weak field black hole formation in asymptotically AdS spacetimes, JHEP 09 (2009) 034 [arXiv:0904.0464] [SPIRES].

[16] P.M. Chesler and L.G. Yaffe, Boost invariant flow, black hole formation and far-from-equilibrium dynamics in $N=4$ supersymmetric Yang-Mills theory, Phys. Rev. D 82 (2010) 026006 [arXiv:0906.4426] [SPIRES].

[17] S.-S. Lee, A non-Fermi liquid from a charged black hole: a critical Fermi ball, Phys. Rev. D 79 (2009) 086006 [arXiv:0809.3402] [SPIRES].

[18] H. Liu, J. McGreevy and D. Vegh, Non-Fermi liquids from holography, arXiv:0903.2477 [SPIRES].

[19] M. Cubrovic, J. Zaanen and K. Schalm, String theory, quantum phase transitions and the emergent Fermi-liquid, Science 325 (2009) 439 [arXiv:0904.1993] [SPIRES].

[20] S.A. Hartnoll, J. Polchinski, E. Silverstein and D. Tong, Towards strange metallic holography, JHEP 04 (2010) 120 [arXiv:0912.1061] [SPIRES].

[21] S.A. Hartnoll and A. Tavanfar, Electron stars for holographic metallic criticality, arXiv: 1008.2828 [SPIRES]. 
[22] J. de Boer, K. Papadodimas and E. Verlinde, Holographic neutron stars, JHEP 10 (2010) 020 [arXiv:0907.2695] [SPIRES].

[23] R. Jost, The general theory of quantized fields, American Mathematical Society, U.S.A. (1965).

[24] T. Banks, M.R. Douglas, G.T. Horowitz and E.J. Martinec, AdS dynamics from conformal field theory, hep-th/9808016 [SPIRES].

[25] O. Aharony, S.S. Gubser, J.M. Maldacena, H. Ooguri and Y. Oz, Large-N field theories, string theory and gravity, Phys. Rept. 323 (2000) 183 [hep-th/9905111] [SPIRES].

[26] E.H. Lieb and W.E. Thirring, Gravitational collapse in quantum mechanics with relativistic kinetic energy, Ann. Phys. 155 (1984) 494 [SPIRES].

[27] E.H. Lieb and H.-T. Yau, The Chandrasekhar theory of stellar collapse as the limit of quantum mechanics, Comm. Math. Phys. 112 (1987) 147 [SPIRES].

[28] R. Ruffini and S. Bonazzola, Systems of selfgravitating particles in general relativity and the concept of an equation of state, Phys. Rev. 187 (1969) 1767 [SPIRES].

[29] R.H. Boyer and R. Lindquist, A variational principle for a rotating relativistic fluid, Phys. Lett. 20 (1966) 504.

[30] J.B. Hartle and D.H. Sharp, Variational principle for the equilibrium of a relativistic, rotating star, Astrophys. J. 147 (1967) 317.

[31] F.E. Schunck and E.W. Mielke, General relativistic boson stars, Class. Quant. Grav. 20 (2003) R301 [arXiv:0801.0307] [SPIRES].

[32] D.N. Page and K.C. Phillips, Selfgravitating radiation in Anti-de Sitter space, Gen. Rel. Grav. 17 (1985) 1029 [SPIRES].

[33] J.A. Wheeler, Geons, Phys. Rev. 97 (1955) 511 [SPIRES].

[34] S. Shapiro and S. Teukolsky, Black holes, white dwarfs and neutron stars: the physics of compact objects, Wiley, U.S.A. (1983).

[35] C.W. Misner, K.S. Thorne and J.A. Wheeler, Gravitation, Freeman, U.S.A. (1973).

[36] J.M. Heinzle, N. Rohr and C. Uggla, Spherically symmetric relativistic stellar structures, Class. Quant. Grav. 20 (2003) 4567 [gr-qc/0304012] [SPIRES].

[37] P.-H. Chavanis, Relativistic stars with a linear equation of state: analogy with classical isothermal spheres and black holes, arXiv:0707.2292 [SPIRES].

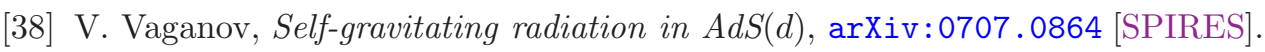

[39] J. Hammersley, A critical dimension for the stability of perfect fluid spheres of radiation, Class. Quant. Grav. 25 (2008) 205010 [SPIRES].

[40] F.A. Dolan and H. Osborn, Conformal four point functions and the operator product expansion, Nucl. Phys. B 599 (2001) 459 [hep-th/0011040] [SPIRES].

[41] E. Witten, Anti-de Sitter space and holography, Adv. Theor. Math. Phys. 2 (1998) 253 [hep-th/9802150] [SPIRES].

[42] L. Álvarez-Gaumé, C. Gomez and M.A. Vazquez-Mozo, Scaling phenomena in gravity from QCD, Phys. Lett. B 649 (2007) 478 [hep-th/0611312] [SPIRES].

[43] L. Álvarez-Gaumé, C. Gomez, A. Sabio Vera, A. Tavanfar and M.A. Vazquez-Mozo, Critical gravitational collapse: towards a holographic understanding of the Regge region,

Nucl. Phys. B 806 (2009) 327 [arXiv:0804.1464] [SPIRES]. 
[44] L. Álvarez-Gaumé, C. Gomez, A. Sabio Vera, A. Tavanfar and M.A. Vazquez-Mozo, Critical formation of trapped surfaces in the collision of gravitational shock waves, JHEP 02 (2009) 009 [arXiv:0811.3969] [SPIRES].

[45] A. Avgoustidis, R. Jimenez, L. Álvarez-Gaumé and M.A. Vazquez-Mozo, Powering AGNs with super-critical black holes, arXiv:0905.2109 [SPIRES].

[46] J. Novak, Velocity-induced collapses of stable neutron stars, Astron. Astrophys. 376 (2001) 606 [gr-qc/0107045] [SPIRES].

[47] S.C. Noble and M.W. Choptuik, Type II critical phenomena of neutron star collapse, Phys. Rev. D 78 (2008) 064059 [arXiv:0709. 3527] [SPIRES].

[48] L.-Y. Hung, D.P. Jatkar and A. Sinha, Non-relativistic metrics from back-reacting fermions, Class. Quant. Grav. 28 (2011) 015013 [arXiv: 1006.3762] [SPIRES]. 